\title{
Unbalance Compensation of AMB Systems with Input Delay: A Truncated Predictor Feedback Based Output Regulation Approach
}

\footnotetext{
A Dissertation

Presented to

the Faculty of the School of Engineering and Applied Science

University of Virginia

In Partial Fulfillment

of the requirements for the Degree

Doctor of Philosophy (Mechanical and Aerospace Engineering)

by

Long Di

December 2016
} 
(c) 2016 Long Di 


\section{Approval Sheet}

This Dissertation is submitted in partial fulfillment of the requirements for the degree of Doctor of Philosophy (Mechanical and Aerospace Engineering)

\section{Long Di}

This Dissertation has been read and approved by the Examining Committee:

Zongli Lin, Advisor

Houston Wood, Committee Chair

Gang Tao

Chris Goyne

Baoxing $\mathrm{Xu}$

Accepted for the School of Engineering and Applied Science:

Craig Benson, Dean, School of Engineering and Applied Science

December 2016 


\section{Abstract}

Active magnetic bearings (AMBs) adopt electromagnetic forces to support the rotating shaft

and do not have physical contact with the rotary structure. Compared to conventional mechanical bearings, AMBs do not require lubrication; the non-contact working environment improves the efficiency and reduces maintenance cost caused by mechanical wear; the dynamic forces also help the machinery to achieve higher rotational speeds. These appealing features of AMBs have gradually expanded their application in different industries, especially those involving high speed rotating machineries, such as compressors, where efficiency and reliability are highly desired.

Emerging applications in offshore drilling for oil and gas production require compressors to operate in a remote and harsh environment for long periods of time. By adopting AMB technologies, these remotely operated applications have become technically and economically more feasible. Motivated by the challenges in those applications, this dissertation first addresses controlling high speed compressors supported by AMBs in offshore oil and gas development, where the control and sensor measurement signals are transmitted through long cables between the control unit on the shore and the compressor on the seabed. These cables, which may extend for several kilometers, introduce significant time delays to the system and the delays can degrade the system performance and even cause instability. Therefore, control methods that effectively contain the delay effect become indispensable. In this dissertation, the truncated predictor feedback (TPF) control law is applied to handle the time delay. The TPF control method utilizes the prediction of future states in the control signal calculation 
to eliminate the delay effect. The controller corresponding to the maximized input delay that the closed-loop system can tolerate is obtained iteratively from the solution of a linear matrix inequality (LMI) problem. It is demonstrated in the dissertation that the TPF controller can tolerate a significant amount of input delay for AMB system levitation and outperforms a properly designed $\mu$-synthesis robust controller.

The second topic of this dissertation is unbalance compensation of AMB systems at both constant and time-varying rotational speeds. Any rotating machines are subject to disturbance forces caused by residual unbalance and the disturbance force is synchronous to the rotational speed. Therefore, unbalance compensation is crucial for reducing rotor vibration and preserving the system stability in high speed rotating machines. A properly controlled compressor supported by AMBs needs to confine the vibration to a small level to satisfy industrial standards. To mitigate the adverse effects of unbalance forces, this dissertation proposes a novel unbalance compensation technique based on the output regulation mechanism. The problem of output regulation is to design a controller for disturbance rejection and/or reference tracking, while the disturbance or reference signal is generated by a known dynamic system called exosystem. For a time-invariant exosystem, the regulation error can be fully eliminated while for a time-varying exosystem, it is observed that a small residual error exists in the regulated output for a non-minimum phase system, such as an AMB system. A unified gradient method is adopted to ensure that the error is small. To apply the output regulation mechanism, the unbalance forces are modeled as the output of the exosystem and the compensator gains are obtained based on the solution of static regulator equations for the constant speed case and differential regulator equations for the time-varying speed case.

Eventually, the TPF control law and the output regulation based unbalance compensation method are combined to achieve the control requirements of a remotely located compressor supported by AMBs at different rotational speeds. To demonstrate the effectiveness and applicability of the proposed methods, extensive simulations and experiments have been performed using precise AMB system models and AMB test rigs. 


\section{Acknowledgments}

I would like to deeply thank my advisor Prof. Zongli Lin for bringing me into ROMAC, providing me the opportunities to work on several exciting projects, continuously stimulating me to pursue research excellence, and mostly importantly, backing me up and inspiring me when I got stuck and faced formidable challenges. Whenever I seek advice or discussion, Prof. Lin is always available no matter whether it is in the middle of the week or during the weekend. Without his guidance and encouragement, I would not have been able to reach the current stage.

I want to express my sincere appreciation to my dissertation committee members, Prof. Houston Wood, Prof. Gang Tao, Prof. Chris Goyne and Prof. Baoxing Xu for their support of my research, revising my dissertation and providing valuable advice.

I am grateful to have Prof. Se Young Yoon and Dr. Parinya Anantachaisilp as my colleagues and friends. I learned much AMB control related knowledge and skills from them and they provided a lot of assistance in the projects I was involved. Besides, they have been sharing their inspiring life and school experiences with me, which motivated me to work harder and become stronger.

I would also like to thank Dr. Simon Mushi for helping me with the ROMAC flexible rotor AMB test rig. I also want to thank ROMAC faculty members and fellow students, Prof. Paul Allaire, Dr. Roger Fittro, Dr. Brian Weaver, Dr. Jason Kaplan, Dr. Ali Gerammi, Day Griffin, Benny Schwartz, Yusheng Wei for sharing their knowledge and discussing with me on topics related to AMB, control design and rotordynamics. I also want to send my 
thanks to Ms. Lori Pedersen for helping me with the school documents and ordering the parts for my experiments.

My PhD journey would have been so lonely without my friends, Dr. Jia Hu, Long Chen, Jing Guo, Cheng Yang, Zhiyuan Tao, Ge Song, Longze Chen, Dr. Jiekun Yang and many others. We came to UVa around the same time and we work together towards the same goal. With their company, I always have someone to share the joy and stress.

My parents are the biggest supporters of my school journey. They provided me the initial opportunity to study in the US and the once a week video chat for the last 11 years since I came here has become a tradition for our family. I am always willing to share my happiness and depression with them and they have been so considerate and supportive throughout my education path. I am so thankful for being raised in the "DI" family.

Last but not least, I want to send my gratitude to my wife May Zou, who has been so patient, thoughtful and accommodating. Without her love and support, this journey would have been more difficult. 


\section{Contents}

Contents vi

List of Figures. . . . . . . . . . . . . . . . . . viii

1 Introduction $\quad 1$

1.1 Problem statement . . . . . . . . . . . . . . . . . . . . 1

1.2 Objectives of the dissertation . . . . . . . . . . . . . . . . . . . . . . . . . . . .

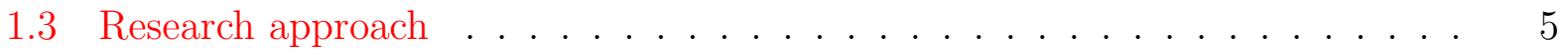

1.4 Research contributions . . . . . . . . . . . . . . . . . . . 7

1.5 Organization of the dissertation . . . . . . . . . . . . . . 7

2 Background and Literature Review 9

2.1 Overview of AMB systems . . . . . . . . . . . . . . . . . . . . . . . . . . .

2.2 AMB control fundamentals . . . . . . . . . . . . . . . . . . . . . . . . . . . . . . . . . . . . .

2.3 Control of systems with time delays . . . . . . . . . . . . . . . . . . . . . . . . . . . . . . . 12

2.4 Vibration control of AMB systems . . . . . . . . . . . . . . 13

3 Description of the Test Rig and Rotordynamic Study 15

3.1 System description . . . . . . . . . . . . . . . . . . . 15

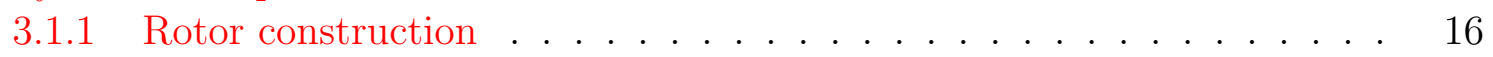

3.1.2 Magnetic bearings . . . . . . . . . . . . . . . . 16

3.1 .3 Electronics. . . . . . . . . . . . . . . . . . . . . . . . . . . . . . . . . . .

3.2 AMB system model . . . . . . . . . . . . . . . . . . . . . . . . . . . . . . . . . . . . . . 20

3.2 .1 Rotor model . . . . . . . . . . . . . . . . . . . . . . . . . . . . . . . . . . . .

3.2.2 Modeling of AMBs . . . . . . . . . . . . . . . . . . . . . . . . . . . . . . . 23

3.2.3 Modeling of additional components . . . . . . . . . . . . . . . 25

3.2.4 The entire AMB system . . . . . . . . . . . . . . . . . . 27

3.3 Summary . . . . . . . . . . . . . . . . . . . 30

4 Fundamentals $\quad 31$

4.1 Control of systems with input delay . . . . . . . . . . . . . . . . . 31

4.2 Output regulation for input-delay system with time-invariant exosystem . . . 34

4.2.1 Output regulation by state feedback . . . . . . . . . . . . . . . 35

4.2.2 Output regulation by output feedback . . . . . . . . . . . . . 37

4.3 Output regulation for input-delay system with time-varying exosystem . . . 39

4.3.1 Output regulation by state feedback . . . . . . . . . . . . 40 
4.3.2 Output regulation by output feedback . . . . . . . . . . . . . . 42

4.3.3 Bounded regulator gains for non-minimum phase systems . . . . . . . 44

4.4 Summary . . . . . . . . . . . . . . . . . . . . . 49

5 Unbalance Compensation under Constant Rotational Speeds $\quad 51$

5.1 TPF control for the AMB test rig . . . . . . . . . . . . . . . . 51

5.1 .1 Maximization of the delay bound . . . . . . . . . . . . . 51

5.1 .2 Controller derivation for the AMB test rig . . . . . . . . . . . 53

5.1 .3 Simulation results . . . . . . . . . . . . . . . 57

5.1 .4 Experimental results . . . . . . . . . . . . . . . . . . . . . . . . . . . . . . . . 58

5.2 Unbalance compensation in AMB systems . . . . . . . . . . . . . . . 63

5.2.1 Problem formulations . . . . . . . . . . . . . . . 64

5.2 .2 Simulation and experimental results . . . . . . . . . . . . . . . . 67

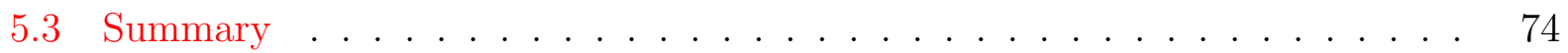

6 Unbalance Compensation under Time-varying Rotational Speeds $\quad 75$

6.1 Autobalancing in an AMB system . . . . . . . . . . . . . . . . . 75

6.1.1 Simulation results . . . . . . . . . . . . . . . . . . . . . . . . . . . . . . . . .

6.2 Unbalance suppression on a balancing beam . . . . . . . . . . . . . 81

6.2.1 Simulation results . . . . . . . . . . . . . . . 86

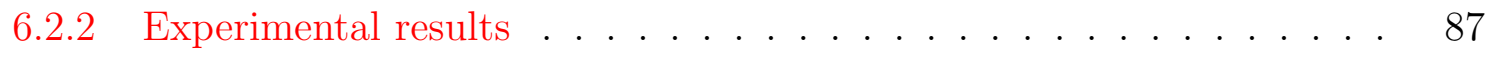

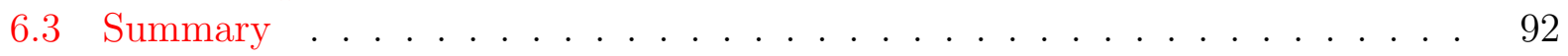

7 Conclusions $\quad 93$

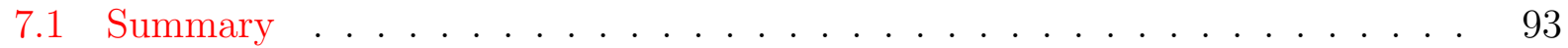

7.2 Future work . . . . . . . . . . . . . . . . . . . . . . . . . . . . . . . . . . . .

$\begin{array}{ll}\text { Bibliography } & 99\end{array}$ 


\section{List of Figures}

1.1 AMB systems in offshore applications. . . . . . . . . . . . . . . 2

1.2 Rotor unbalance. . . . . . . . . . . . . . . . . . . . . . . . . 4

2.1 AMB system operating principle. . . . . . . . . . . . . . . . . 10

3.1 Schematic of the flexible rotor AMB test rig. . . . . . . . . . . . . 16

3.2 The ROMAC flexible rotor AMB test rig. . . . . . . . . . . . . . . . . 17

3.3 A functional overview of the support AMBs. . . . . . . . . . . . . 18

3.4 A functional overview of the exciter AMBs. . . . . . . . . . . . . . . . 19

3.5 AMB system block diagram. . . . . . . . . . . . . . . . . . . . 20

3.6 The flexible rotor finite element model. . . . . . . . . . . . . . . . . . . 21

3.7 The mode shape plot of the rotor. . . . . . . . . . . . . . . . . . 22

3.8 The Campbell diagram of the rotor with $5 \mathrm{MN} / \mathrm{m}$ support stiffness to show the splitting of the natural frequencies with the speed increase. . . . . . . . . 24

3.9 One DOF AMB model. . . . . . . . . . . . . . . . . . . . . . . . . . 24

3.10 Bode plots for the test rig: analytic model vs experimental measurements. . 29

4.1 The unified gradient method process. . . . . . . . . . . . . . 50

5.1 The LMI-based optimization procedure for determining the maximal allowable delay bound. . . . . . . . . . . . . . . . . . . . . 54

5.2 Path of the optimal solution. . . . . . . . . . . . . . . 55

5.3 Singular value plot of the derived output feedback TPF controller. . . . . . . 56

5.4 Simulated step response of the closed-loop AMB system with a constant input delay. . . . . . . . . . . . . . . . . . . . . . . 57

5.5 Simulated zero-to-peak rotor vibration level for rotational speeds up to $7000 \mathrm{rpm} .58$

5.6 Controller feedback loop implementation in the AMB test rig. . . . . . . . . 59

5.7 Rotor displacement measurement during levitation with $\mu$-synthesis controller and input delayed by $D=3 T_{\mathrm{s}} \ldots \ldots \ldots \ldots \ldots \ldots$

5.8 Rotor displacement measurement during levitation with TPF controller and input delayed by $D=3 T_{\mathrm{s}} \ldots \ldots \ldots \ldots \ldots \ldots \ldots$

5.9 Rotor displacement measurement during levitation with TPF controller and input delayed by $D=8 T_{\mathrm{s}} \ldots \ldots \ldots \ldots$. . . . . . . . . . 61

5.10 Rotor displacement measurement during levitation with TPF controller and input delayed by $D=10 T_{\mathrm{s}} \ldots \ldots \ldots \ldots \ldots \ldots$ 
5.11 Zero-to-peak rotor vibration amplitude measured at different rotating speeds with TPF controller and input delayed by $D=6 T_{\mathrm{s}} \ldots \ldots$. . . . . . . . . 63

5.12 Block diagram of the controller implementation under constant rotational speed. 67

5.13 Simulated rotor displacements and AMB forces under the rotor displacement regulation with the output regulator active for $t>1 \mathrm{~s} . \ldots . . . . . .68$

5.14 Simulated rotor displacements and AMB forces under the AMB force regulation

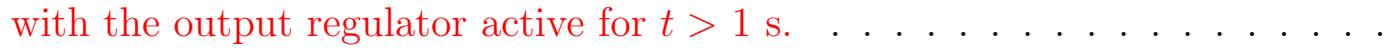

5.15 Experimental measurements under the controller designed for the non-regulated system. . . . . . . . . . . . . . . . . . . .

5.16 Experimental measurements under the controller with the rotor displacement regulation. . . . . . . . . . . . . . . . . . . . . . 71

5.17 Experimental measurements under the controller with the AMB force regulation. 73

6.1 Rotor rigid body modes. . . . . . . . . . . . . . . . . 76

6.2 Block diagram of the controller implementation under time-varying rotational speed. . . . . . . . . . . . . . . . . . . . . . 78

6.3 Simulated control voltages without (left) and with (right) the differential regulator as the rotating speed varies from 1,000 to 7,500 rpm using state feedback. . . . . . . . . . . . . . . . . . . . .

6.4 Simulated control voltages and rotor displacements without (left) and with (right) the differential regulator at 7,500 rpm using state feedback. . . . . . .

6.5 Simulated control voltages without (left) and with (right) the differential regulator as the rotating speed varies from 1,000 to 7,500 rpm using output feedback. . . . . . . . . . . . . . . . . . . .

6.6 Simulated control voltages and rotor displacements without (left) and with (right) the differential regulator at 7,500 rpm using output feedback. . . . . .

6.7 Simulated control voltages and rotor displacements with the differential regulator turned on at 2 seconds. . . . . . . . . . . . . . . . . . . . . . . . 82

6.8 The balancing beam test rig. . . . . . . . . . . . . . . . . . 83

6.9 Illustrative diagram of the balancing beam test rig. . . . . . . . . . . . . 85

6.10 Simulated control current and titling angle without the differential regulator at $980 \mathrm{rpm} \ldots \ldots \ldots \ldots \ldots \ldots$

6.11 Simulated control current and titling angle with the differential regulator at $980 \mathrm{rpm} . \ldots \ldots \ldots \ldots \ldots . \ldots \ldots$

6.12 Simulated control current and titling angle without the differential regulator

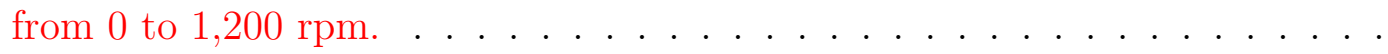

6.13 Simulated control current and titling angle with the differential regulator from 0 to $1,200 \mathrm{rpm} \ldots \ldots \ldots \ldots \ldots$

6.14 Experimental performance without (left column) and with (right column) the

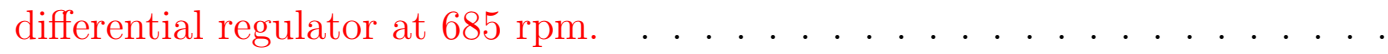

6.15 Experimental performance without (blue curves) and with (red curves) the differential regulator from 0 to $685 \mathrm{rpm}$ and then back to $0 \mathrm{rpm} . . . . .$. 


\section{Chapter 1}

\section{Introduction}

The research and development of active magnetic bearings (AMBs) can be traced back several decades and AMBs have seen steady popularity growth in the industrial applications, ranging from small turbomolecular pumps to large megawatt-level compressors [1]. Compared to conventional mechanical bearings, AMBs adopt different operating principles and retain different structures. On the one hand, AMBs utilize magnetic forces generated by electrical coils to support the shaft so there is no physical contact between the rotating and static components, which creates a nearly friction-free working environment. On the other hand, since AMBs are inherently unstable systems, feedback controllers are indispensable for AMBs to generate the desired dynamic force, which entails additional electronics. Given that AMB supported rotating machines can deliver high efficiency and reliability with low maintenance and repairing costs, they become more suitable for a variety of high speed compressor applications.

\subsection{Problem statement}

Because of their prominent advantages and features, AMB supported rotating machinery has gradually gained popularity in applications where the machines have to be operated remotely. More specifically, emerging applications in offshore drilling for oil and gas production 


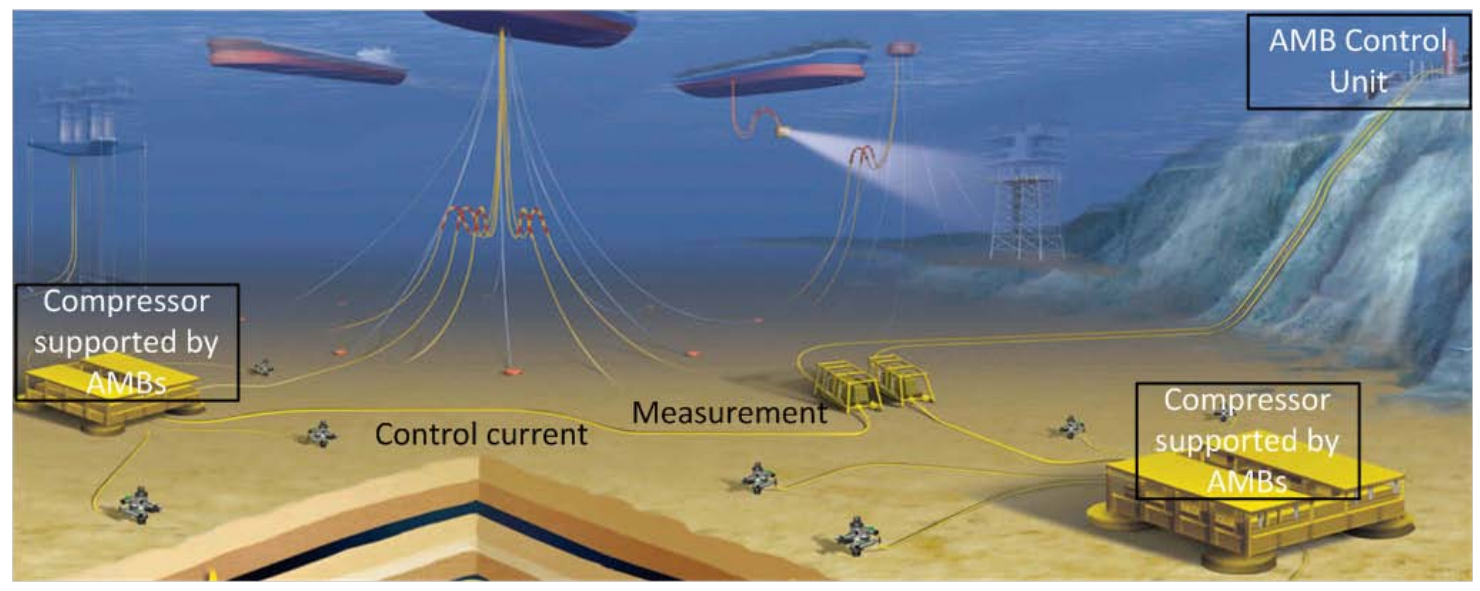

Figure 1.1: AMB systems in offshore applications.

entail compressors to operate in remote and harsh areas for long hours and those areas are not easily accessible for frequent diagnosis and maintenance. By using AMB technologies, these applications have become technically and economically more feasible. Because of the sensitivity concern of the power electronics to the seawater and the challenge in accessing remote compressor sites, manufacturers commonly install AMB control units, which include all the power electronics, on the shores while planting the compressors supported by AMBs on the seabed, as shown in Fig. 1.1.

The AMB control units and AMB actuators are connected through long cables that can extend for several kilometers, which introduces significant time delays that may degrade the performance of the operation and even cause instability. Time delays widely exist in engineering practice, including the gas/fluid flow in piping for chemical processes, computational time in complex control algorithm implementation, and remote control and operation. The amount of delay added by the cabling can be estimated from their physical properties, or directly measured when the cables are accessible. Electrical wire is typically made from copper, and the transmission speed generally ranges from $0.59 c$ to $0.77 c$ [2], where $c$ is the speed of light. For a cable that extends for 20 kilometers, the propagation delay could be as much as 0.113 ms, which is close to the phase margin created by a properly designed controller. In a later chapter, it is shown that an input delay above $0.16 \mathrm{~ms}$ can induce instability in the system. 
When system failures happen, it causes undesired machine downtime for the manufacturers and leads to significant financial loss. Therefore, the control of AMB systems with input delay needs to be carefully investigated.

In addition to the effect of time delay, high speed rotating machines in remote applications are also subject to unbalance forces caused by residual rotor unbalance. Any newly machined rotor always carries some small residual weights and when the rotor's axis of geometry is not aligned with its principal axis of inertia as shown in Fig. 1.2, disturbance forces synchronous to the rotational speed cause the rotor to deflect from the geometric center and enter a whirling motion. Natural vibrations of a rotating shaft usually manifested themselves as a whirling about the rotor axis. In a forward whirl, the whirling motion moves in the same direction as the rotor spinning and, as the unbalance rotates with the rotor spinning, the natural frequencies are excited, leading to resonance. Besides exciting undesirable synchronous vibrations, rotor unbalance can also cause saturation of the amplifiers, result in rotor position runout, and increase the housing vibrations and noise emissions.

The conventional approach to handling rotor unbalance is through installing or removing a small amount of mass from the rotor to counteract the residual unbalance. Nevertheless, this mechanical approach requires a significant amount of experience and is a time-consuming procedure. In addition, for some high speed rotating machines, the unbalance might change during the operation, so mechanical balancing has to be performed repeatedly, causing additional inconvenience. One of the prominent AMB features is that, instead of rotating around the rotor's geometric axis, the shaft can rotate around the principle axis of inertia. Therefore, the unbalance is effectively compensated, and to achieve such balancing-free operation, it requires certain knowledge of the controller design and analysis of unbalance forces.

In order to achieve the control requirement of a remotely located compressor supported by AMBs at different rotational speed conditions, a special advanced control method needs to be developed to handle the adverse effect of the time delay and to minimize the unbalance 


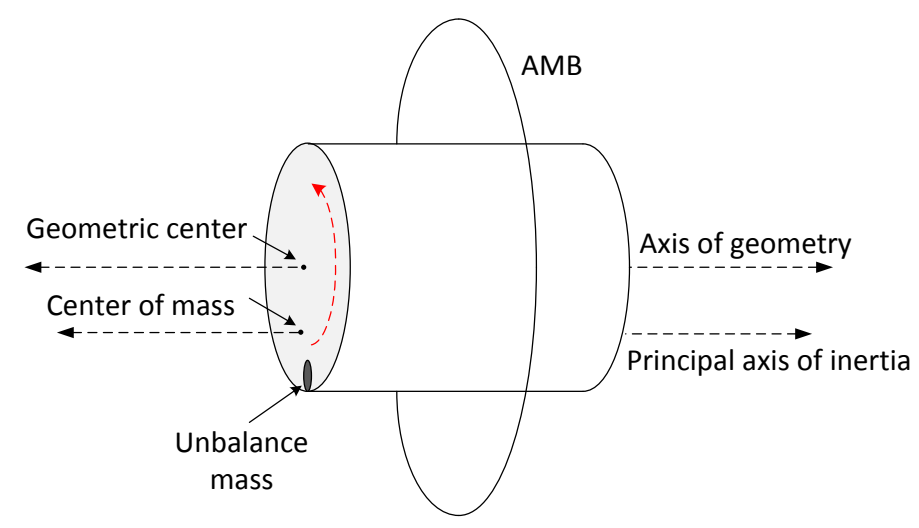

Figure 1.2: Rotor unbalance.

disturbance.

\subsection{Objectives of the dissertation}

Few works have considered the problems of controlling AMB systems with time delays and under different rotating conditions. This dissertation aims to achieve the following objectives:

1. Analyze the rotordynamics of a high-speed flexible rotor AMB test rig that was built to emulate the characteristics of a small industrial centrifugal compressor, develop a comprehensive AMB system modeling program for model-based controller designs, and conduct novel rotordynamic studies using the test rig.

2. Design advanced controllers to stabilize AMB systems subject to input delay and maximize the allowable delay. Implement the algorithms on the AMB test rigs to verify the effectiveness of the proposed method.

3. Develop a new unbalance compensation strategy for time-delay AMB systems under different rotational speed conditions. Implement and assess the proposed method on the AMB test rigs.

4. Compare and evaluate the results from simulation and experimental measurements. 


\subsection{Research approach}

The control of dynamic systems with time delay is an active area of research and the predictor feedback control approach for systems with input delays has been extensively studied in the literature. The predictor feedback control adopts prediction of the future state in calculating the control signal to cancel the effect of the input delay. By using the prediction of the future state, the delayed system becomes an equivalent delay-free system. In recent years, a finite-dimensional predictor by truncating the distributed term of the original predictor equation has been developed for stable and unstable linear systems, which is called the truncated predictor feedback (TPF) control law [3, 4].

The TPF control law has been applied to an AMB system as the rotor levitation controller, which is open-loop exponentially unstable. The controller corresponding to the maximized input delay that the closed-loop system can tolerate is obtained iteratively from the solution of a linear matrix inequality (LMI) problem. The Rotating Machinery and Control (ROMAC) Lab flexible rotor AMB test rig is used for numerical verification and experimental validation of the developed TPF control law.

To reduce the effect that the rotor unbalance has on high speed rotating machinery supported by AMBs, a variety of unbalance compensation methods have been developed. If designed and implemented properly, these methods can significantly mitigate the unbalance forces acting on the rotor. The vibrations due to the residual unbalance are measured by sensors and through different control algorithms, the AMBs can either generate counteracting bearing forces or shift the rotor axis in such a way that the shaft is rotating about its principal axis of inertia to cancel the disturbance forces caused by the unbalance. Thus, the synchronous vibrations are not transmitted to the machine foundations in spite of the presence of unbalance.

Unbalance compensation methods have not been extended to AMB systems subject to time delays. To address this challenge, with the TPF control law serving as the feedback controller, an unbalance compensation method based on the solution of an equivalent output 
regulation problem at a constant rotational speed is developed. The problem of output regulation is to design a controller for disturbance rejection and/or reference tracking, while the disturbance or reference is generated by a known dynamic system called exosystem. Since the rotational speed is constant, the exosystem is time-invariant. By applying output regulation to AMB systems for unbalance compensation, the unbalance force is modeled by the exosystem and the control voltage defines the error to be regulated. The resulting model based controller will demonstrate that both synchronous rotor vibrations and the magnitude of control output are significantly reduced.

After considering the case of constant rotational speed, the research is extended to the autobalancing and unbalance suppression problems of AMB systems subject to input delay at time-varying rotational speeds. The speed generally varies slowly since the torques of the drive and the load are limited. To reject the unbalance disturbance under different rotational speed conditions, the main approach discussed in the literature is to intermittently switch the regulator gains that have been designed beforehand and saved in the memory as a look-up table, similar to the concept of gain scheduling control. Switching of regulator gains may result in bumpy transition. Therefore, this approach is not only cumbersome but also restricted to discrete speed ranges.

To address the remaining challenge in the research, a differential regulator based output regulation approach is presented to address the unbalance mitigation problem of AMB systems for varying rotational speeds. After formulating the output regulation problem with a time-varying exosystem, it is observed that the compensator gains can be obtained based on the solution of the differential regulator equation (DRE) and can be iteratively generated to closely approach the output regulation objective with a small bounded residual error in the regulated output. When the rotational speed changes in an AMB system, the exosystem derived from the unbalance forces becomes time-varying, and the proposed differential regulator based output regulation approach can be adopted to generate the desired compensator gains that locally minimize the AMB control force and suppress the vibration. 
The resulting compensator will demonstrate those capabilities at varying rotational speeds.

\subsection{Research contributions}

The main contributions of this dissertation are summarized as follows:

1. Develop a truncated predictor feedback based output regulation mechanism to control AMB supported high speed compressors in remotely operated applications, given that the unbalance compensation of AMB systems subject to time delay has not been documented in the previous literature.

2. Derive a practical differential regulator based output regulation algorithm to achieve locally minimized residual error in the regulated output for non-minimum phase systems that are disturbed by the signals generated from time-varying exosystems, considering that little literature has addressed such a problem and the current approach has restrictions.

3. Design and implement the control algorithms on the AMB test rigs to demonstrate the effectiveness of the proposed method.

4. Characterize how small an input delay can affect the system stability and achieve the maximization of the delay bound using the LMI optimization method.

5. Demonstrate the versatility of the output regulation framework by investigating different output regulation scenarios that achieve unbalance suppression.

\subsection{Organization of the dissertation}

The remainder of the dissertation is organized as follows: 
1. Chapter 2 briefly describes the operating principles of AMBs and give a brief review of the literature on the control of systems with time delay and on unbalance compensation of AMB systems.

2. Chapter 3 describes the flexible rotor AMB test rig that is used for the experimental validation and presents the AMB system modeling procedure.

3. Chapter 4 details the fundamentals of this dissertation. The TPF control law is first introduced. Then the output regulation with time-invariant and time-varying exosystems are formulated.

4. Chapter 5 presents the unbalance compensation for the time-delay AMB system operating at a constant rotational speed. The TPF controller for the flexible rotor AMB test rig is first derived to achieve the maximization of the allowable delay bound by using the LMI method. Then experimental tests are conducted and the TPF controller is compared with a $\mu$-synthesis robust controller. Afterwards, the unbalance compensation with input delay using the output regulation mechanism is considered and two scenarios of output regulations are investigated in both simulation and experiments on the flexible rotor AMB test rig.

5. Chapter 6 presents the unbalance compensation for time-delay AMB systems under time-varying rotational speeds. It starts with autobalancing of AMB systems at continuously changing unbalance disturbance. The TPF control law and the differential regulator based output regulation method are applied to fulfill the unbalance suppression requirement. The flexible rotor AMB test rig and a balancing beam test rig are adopted to verify the proposed compensation method with extensive simulation and experimental results.

6. Chapter 7 summarizes the work completed in the dissertation and provides suggestions for the future research. 


\section{Chapter 2}

\section{Background and Literature Review}

\subsection{Overview of AMB systems}

Active magnetic bearings (AMBs) require feedback controllers to generate appropriate electromagnetic forces to stabilize rotating components, so they entail additional electronic devices, such as power amplifiers, sensors and digital computers. Fig. 2.1 illustrates the basic operating principle of an AMB system in one degree of freedom: when the perturbation currents $\pm i_{c}$ converted by power amplifiers are fed to the copper coils wrapped around the poles in the bearing casing, the electromagnets produce dynamic forces to adjust the movement of the rotor and try to maintain it near the geometric center of the AMB. Whenever the rotating component deflects, displacement sensors capture the instantaneous changes $x$ and pass the measurement information to the controller. The feedback controller then

produces suitable voltage that drives the coils to generate counteracting forces $f_{\text {amb }}$ so that the rotor always remains in the allowable clearance in the AMB.

Compared to mechanical bearings, AMBs possess several advantages but are also more complex and introduce new challenges. Because of the nonlinearity and instability of AMBs, control design is the most essential task in an AMB application. 


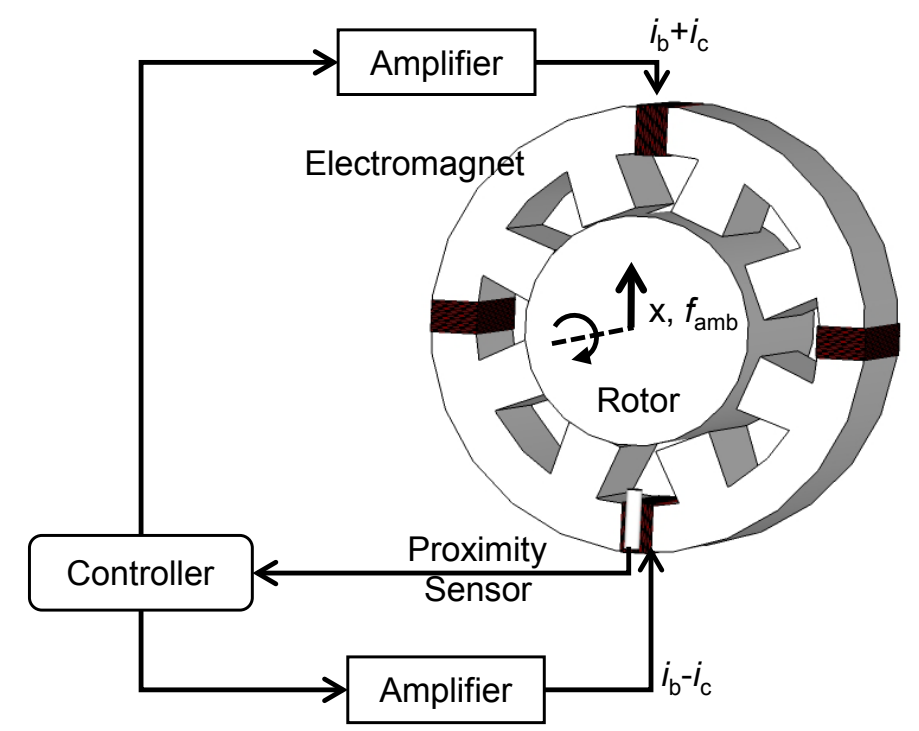

Figure 2.1: AMB system operating principle.

\subsection{AMB control fundamentals}

The AMB system is inherently open loop unstable and a well designed feedback controller becomes indispensable for the closed loop system stability and smooth operation. There are several factors that create challenges to the control design of AMB systems, including flexibility of structures or rotors, unbalance forces that are synchronous to the rotational speed, nonlinear dynamics of magnetic bearings, and sensitivity to changes in AMB components.

Proportional-integral-derivative (PID) controllers are the most widely used control mechanism for AMB related industrial applications. Because of their simple structure, PID controllers are easy to implement and can be tuned intuitively. They can achieve reasonable control performance with proper tuning of the parameters. However, for complex dynamic systems, such as AMB supported flexible rotors, it is difficult for PID controllers alone to provide sufficient control authority and to deliver robust performance without additional filters. When the system parameters change or the working condition varies, PID controllers might need to be retuned to ensure the AMB system stability and performance requirements.

Given the deficiencies of PID controllers, advanced controllers are needed to push the envelop and different approaches have been applied to stabilize AMB systems with improved 
performance and robustness. These approaches are primarily based on modern control theory. These control designs rely on precise system dynamic models and usually possess multipleinput multiple-output (MIMO) structures. Some typical designs include linear quadratic Gaussian (LQG) control, $H_{\infty}$ control and $\mu$-synthesis control [5]. These methods rely on extensive mathematics to optimize the control performance, although they are not as familiar as PID controllers to most practitioners. Another challenge of model-based control is that the designs are sensitive to model uncertainties. For AMB systems, there are several major causes of model uncertainties, including unmodeled high-frequency dynamics, resonant frequency splitting caused by the gyroscopic effect, and rotor modal damping variations.

Since advanced control methods typically require preliminary work on system modeling and model validation, their initial commissioning time is longer than PID controllers. However, a well developed model-based controllers can generally reach the control design criteria and sometimes even exceed them. A typical way to design controllers for AMB systems is to start with a simple PID controller for leviation and initiate the preliminary modeling and validation procedures. Then the practitioner can rely more on the advanced control methods and conduct systematic design to arrive at a final controller.

Besides those linear control approaches, other advanced design methods, such as adaptive control, have also been applied to AMB systems. A recent work [6] introduces a characteristic model based all-coefficient adaptive control to AMB supported high speed rotating machineries. This method does not require the actual system model. It relies on the characteristic model, described by a second order time-varying difference equation, and uses a gradient adaptive algorithm to generate estimates of the characteristic parameters, which are then used as the indirect adaptive control parameters. Moreover, the adaptive control method still preserves a relatively simple structure that makes it easy to implement. It has demonstrated comparable performance and robustness over some well-known robust controllers. 


\subsection{Control of systems with time delays}

The control of dynamic systems with time delay is an active area of research. Recent developments in this area, as well as some open research problems, are discussed in detail in $[7,8]$. More detailed discussions of different control solutions for the stabilization of linear time-delay systems are presented in [9]. References [10] and [11] study the control of time-delay systems with input saturation, while [12], [13] and [14] develop different solutions for robust stabilization of time-delay systems with model uncertainties. On the application side, the effect of time-delay has been considered, for examples, in robotics [15], autonomous vehicles [16], and magnetic levitation [17].

The predictor feedback control approach for systems with input delay has been studied extensively in the literature since the Smith predictor was introduced in [18]. In the predictor feedback control, a prediction of the future states is utilized in the computation of the control signal to cancel out the effect of the input delay. By utilizing the state prediction in generating the control output, the delay system is transformed into an equivalent delay-free system. A vast majority of the predictor feedback methods for linear systems found in the literature is based on the Artstein model reduction technique [19] and the finite spectrum assignment technique [20].

Variations of these predictor feedback control methods have been presented in the literature in recent years. Predictor based controllers have been constructed for uncertain Euler-Lagrange systems [21], for linear systems with time-varying delays [22], and for systems with unknown input delays [23], to name a few. A finite dimensional predictor, which is obtained by truncating the distributed term of the original predictor equation, was developed in [3] for linear systems with all poles in the closed left-half plane. The truncated predictor relies on the low gain feedback design [24] to ensure the stability of the closed-loop system. This finite dimensional predictor was later expanded in [4] to systems with time-varying delays, and in [25] to exponentially unstable systems. 


\subsection{Vibration control of AMB systems}

High speed rotating machines are susceptible to large disturbance forces caused by the rotor unbalance. Residual unbalance on a rotor can generate disturbance forces synchronous to the rotating speed, causing the rotor to go into a whirling motion. To reduce the rotor unbalance effect on AMB systems, various unbalance compensation methods have been investigated and developed over the years. These methods can significantly reduce the disturbance forces acting on the rotor. The interest in rotor unbalance suppression methods has increased rapidly in recent years as high speed AMB applications become more common. A small sample of the literature studying the unbalance compensation problem can be found in [26-33] and the references therein.

Reference [26] presents one of the earliest and most commonly used unbalance compensation techniques for AMB systems. In [26], generalized notch filters are designed and inserted into the system to cancel the synchronous signal components while the controller design remains unrestricted and the closed-loop stability is preserved. However, the notch filter can still potentially jeopardize the stability margin of the system due to the associated changes in the phase response. The feedforward unbalance compensation scheme is another common approach. It generates a sinusoidal compensation signal which has the appropriate magnitude and phase information to cancel out the corresponding sinusoidal component in the sensor measurement signal. Shafai et al. presents an adaptive forced balancing (AFB) method to address the problem of synchronous vibration caused by mass unbalance in AMB systems [27]. The AFB computes a synchronous reference signal and inserts it at the reference adding location to cancel the synchronous component of the system output. In [30], a time-domain iterative learning control (ILC) and gain-scheduled control based unbalance compensation method is proposed. The ILC scheme is able to work in a predefined speed range and the control gain is automatically adjusted through learning to compensate for different speeds. Reference [31] applies the multivariable $H_{\infty}$ control technique to an AMB supported rigid rotor. It is shown that the $H_{\infty}$ controller, which adopts different structures of weighting func- 
tions and uncertain parametric structure for the bearing stiffness and rotor speed, prevents the whirling of the rotor at the critical speed and achieves substantial vibration suppression of the unbalance. Tang et al. build a magnetically suspended flywheel and propose notch filter based open-loop and closed-loop control methods to eliminate the vibration caused by the residual unbalance [32]. Their compensation method utilizes adaptive notch filters to generate additional currents which have equivalent magnitudes and opposite phases to eliminate the synchronous components in the control current. Reference [33] proposes a double-loop design to compensate the unbalance vibration. They first identify the rotor unbalance characteristic using generalized notch filters, and then apply a feedforward loop at low speeds and an adaptive tuning loop at high speeds. The only drawback of their method is that the synchronous magnetic bearing force always exists, which is mainly caused by the measurement error and variations of the AMB current stiffness and displacement stiffness.

Throughout the literature, most unbalance compensation methods focus on constant rotational speeds and have not been extended to AMB systems that are subjected to time delay. 


\section{Chapter 3}

\section{Description of the Test Rig and Rotordynamic Study}

In this chapter, the ROMAC flexible rotor AMB test rig that is to be used to validate the proposed control method is described in detail. Then the models for the major components in the AMB system are enumerated and the procedure of formulating the state space model for the entire AMB system is described.

\subsection{System description}

The flexible rotor AMB test rig is a research platform constructed in the ROMAC laboratory. The original purpose of this test rig was to emulate an industrial size centrifugal gas compressor and perform advanced control designs $[5,6]$. A schematic of the rotor AMB test rig is shown in Fig. 3.1. In particular, Disk 1 and Disk 2 emulate the wheels in a compressor. There are four AMBs in the test rig. Two radial support AMBs are located at the non-driven end (NDE) and the driven end (DE) of the rotor. One exciter AMB is at the mid span and the other is at the quarter span of the rotor. This combination of four radial AMBs allows the simulation of different operating conditions of a compressor. 


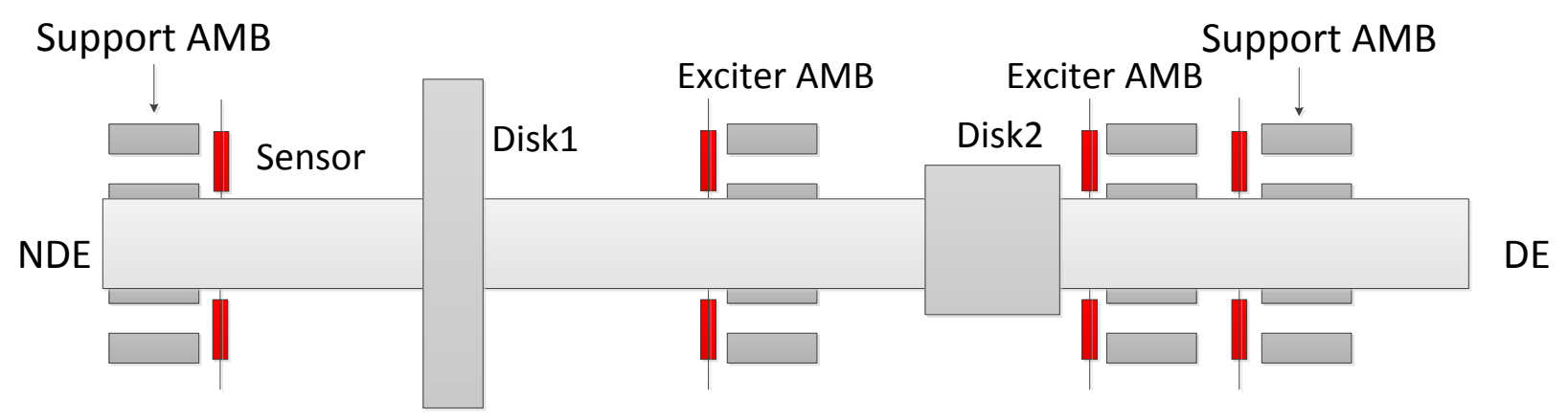

Figure 3.1: Schematic of the flexible rotor AMB test rig.

\subsubsection{Rotor construction}

The rotor in the test rig is $1.23 \mathrm{~m}$ long and weights $44.9 \mathrm{~kg}$. Four laminated steel journals are mounted on the shaft respectively for the two radial support AMBs at the NDE and the DE locations, and the two radial exciter AMBs at the rotor mid and quarter spans. There are also two auxiliary ball bearings mounted at the support AMB locations to prevent damage to the AMBs in the event of a rotor drop. A $3.7 \mathrm{~kW}$ high speed motor with variable frequency drive (VFD), Colombo RS-90/2, drives the rotor in the test rig to speed up to 18,000 rpm. The drive is connected to one end of a flexible disc coupling using a custom shaft extension. The assembled flexible rotor AMB test rig is shown in Fig. 3.2.

\subsubsection{Magnetic bearings}

The two support AMBs utilize a 16-pole heteropolar design with M-15 Si-Fe lamination. There are 48 turns of \#18 copper wires for each pole. The air gaps for the NDE and DE AMBs are $0.518 \mathrm{~mm}$ and $0.593 \mathrm{~mm}$, respectively. The quadrant inductance $L_{w}=12 \mathrm{mH}$ and the quadrant resistance $R_{w}=0.35 \Omega$. The two exciter AMBs utilize a 8-pole design and there are 94 turns of \#18 copper wires for each pole. The air gaps for the mid and quarter span AMBs are $0.575 \mathrm{~mm}$ and $0.491 \mathrm{~mm}$, respectively. The quadrant inductance $L_{w}=36 \mathrm{mH}$ and the quadrant resistance $R_{w}=0.34 \Omega$. The quadrant orientation has a $45^{\circ}$ offset from the vertical axis so that the rotor weight is evenly distributed between the two control channels, 


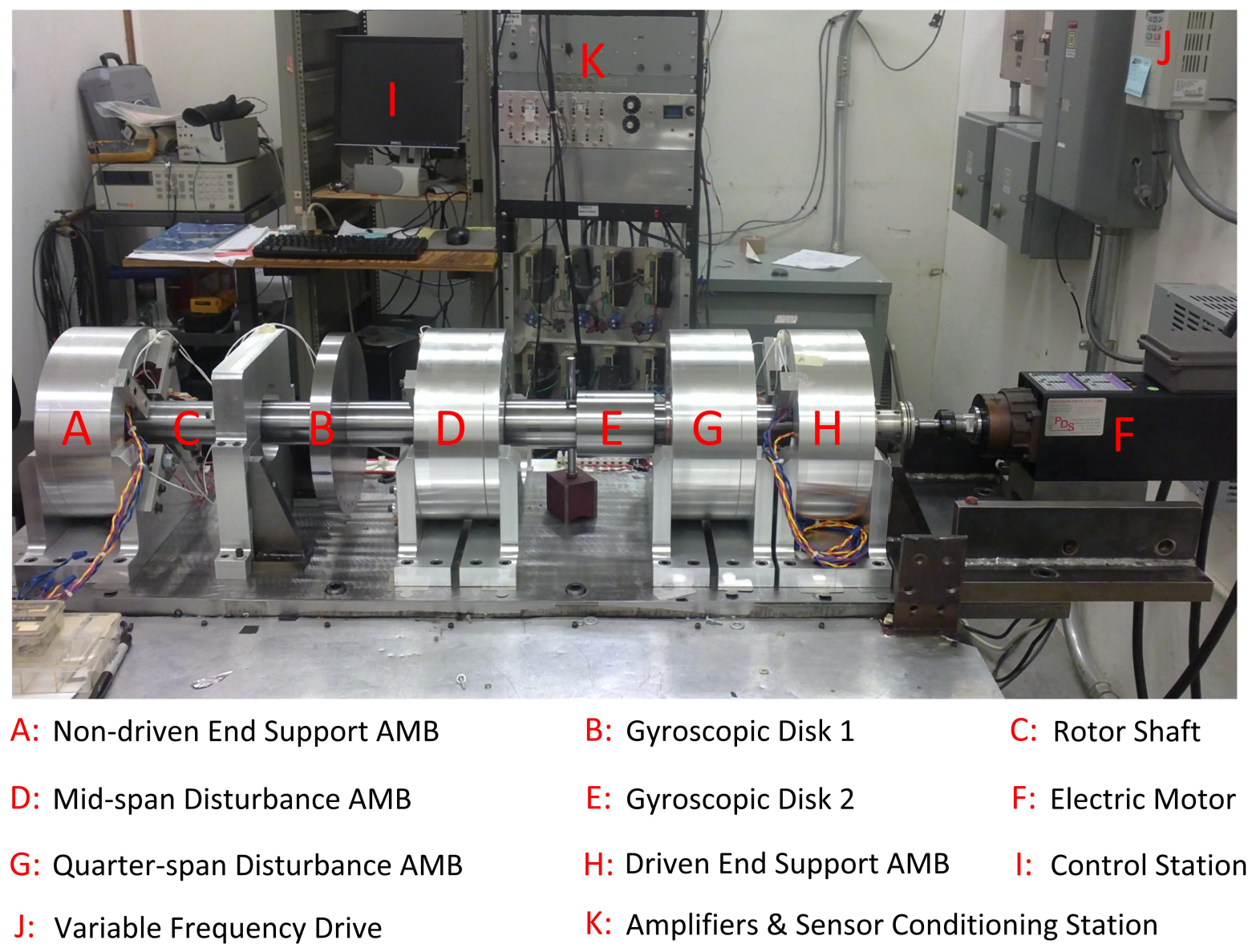

Figure 3.2: The ROMAC flexible rotor AMB test rig.

which also ensures the $x$ and $y$ axes possess identical rotordynamics. The properties of the support and exciter AMBs are summarized in Table 3.1.

\subsubsection{Electronics}

The digital control system is based on an Innovative Integration M6713 PCI board and a TI C6713B 32-bit floating point digital signal processing (DSP) chip is used for the implementation of the digital control algorithm with an updating frequency of $12 \mathrm{kHz}$. Sixteen input-output analog channels are simultaneously sampled and interfaced with the 16 sensors and the 16 actuators associated with the four AMBs.

A functional overview of the support AMBs is shown in Fig. 3.3. The pair of the support AMBs are driven by a Model 422 analog PWM amplifier from Copley Controls operating 


\begin{tabular}{|l|c|c|c|}
\hline Property & NDE/DE & mid/quarter & Units \\
\hline Bias current, $I_{\mathrm{b}}$ & 4 & 1 & $\mathrm{~A}$ \\
\hline Number of poles, $n$ & 16 & 8 & -- \\
\hline Estimated air gap flux density, $B$ & $0.55 / 0.5$ & 0.27 & $\mathrm{~T}$ \\
\hline Current gain, $K_{\mathrm{i}}$ & $130 / 100$ & $94 / 91$ & $\mathrm{~N} / \mathrm{A}$ \\
\hline Negative stiffness, $K_{\mathrm{x}}$ & $900 / 600$ & $165 / 186$ & $\mathrm{kN} / \mathrm{m}$ \\
\hline Copper turns, $N$ & 48 & 94 & -- \\
\hline Projected pole area, $A_{\mathrm{p}}$ & 348 & 700 & $\mathrm{~mm}^{2}$ \\
\hline Nominal air gap, $g_{0}$ & $0.518 / 0.593$ & $0.575 / 0.491$ & $\mathrm{~mm}$ \\
\hline Stator axial length, $l$ & 43.6 & 43.6 & $\mathrm{~mm}$ \\
\hline Stator outer diameter, $D_{0}$ & 196 & 196 & $\mathrm{~mm}$ \\
\hline Stator inner diameter, $D_{i}$ & $92.412 / 92.424$ & $92.418 / 92.358$ & $\mathrm{~mm}$ \\
\hline Rotor lamination diameter, $D$ & $91.377 / 91.237$ & $91.269 / 91.377$ & $\mathrm{~mm}$ \\
\hline Back iron thickness, $T_{b}$ & 0.0171 & 0.0201 & $\mathrm{~mm}$ \\
\hline Stator weight & 35.6 & 53.4 & $\mathrm{~N}$ \\
\hline
\end{tabular}

Table 3.1: Support and exciter AMBs properties.

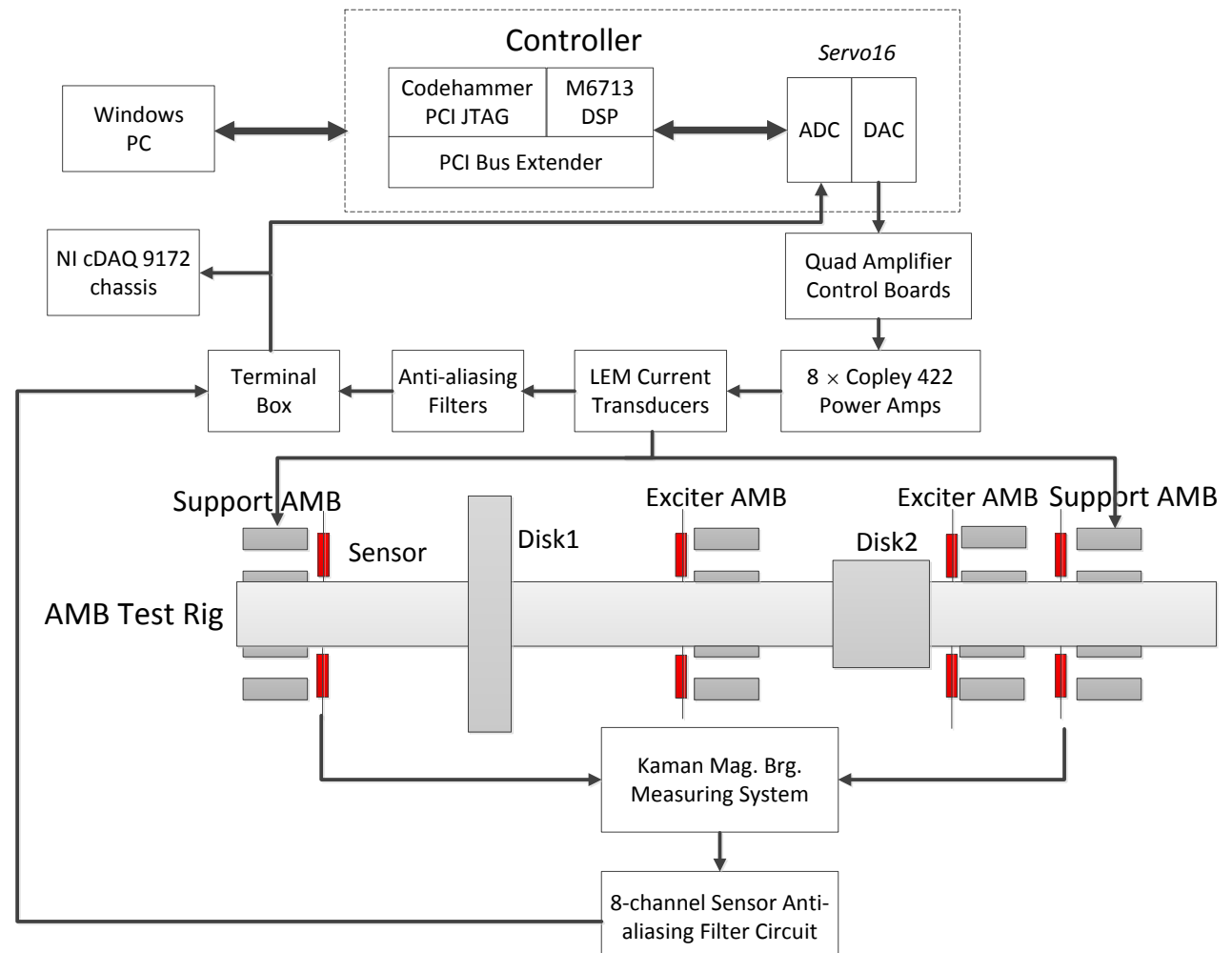

Figure 3.3: A functional overview of the support AMBs. 
from a $150 \mathrm{~V}$ DC supply with a 10 A continuous current rating, which gives each AMB a static load capacity of $1450 \mathrm{~N}$. The rotor motion at each support AMB location is detected using a Kaman eddy current probe measuring system. A pair of Kaman 1H/15N static probes are mounted to measure the displacements of the two control axes. The high frequency noise of the displacement signals is attenuated by an anti-aliasing filter circuit.

A functional overview of the exciter AMBs is shown in Fig. 3.4. The mid span and the quarter span exciter AMBs are driven by four Copley Controls 413 and 4212 amplifiers. A pair of Bently Nevada 7200 Series eddy current probes are used at the mid span and quarter span locations. A gain/offset circuit is connected to a BN 7200 Series proximitor, changing the output voltage from around $-10 \mathrm{~V}$ to $0 \mathrm{~V}$. The two AMBs in the mid and quarter spans have been used to emulate the negative stiffness of the generator and the gyroscopic effects of the flywheel disk on the rotordynamics in the recent research.

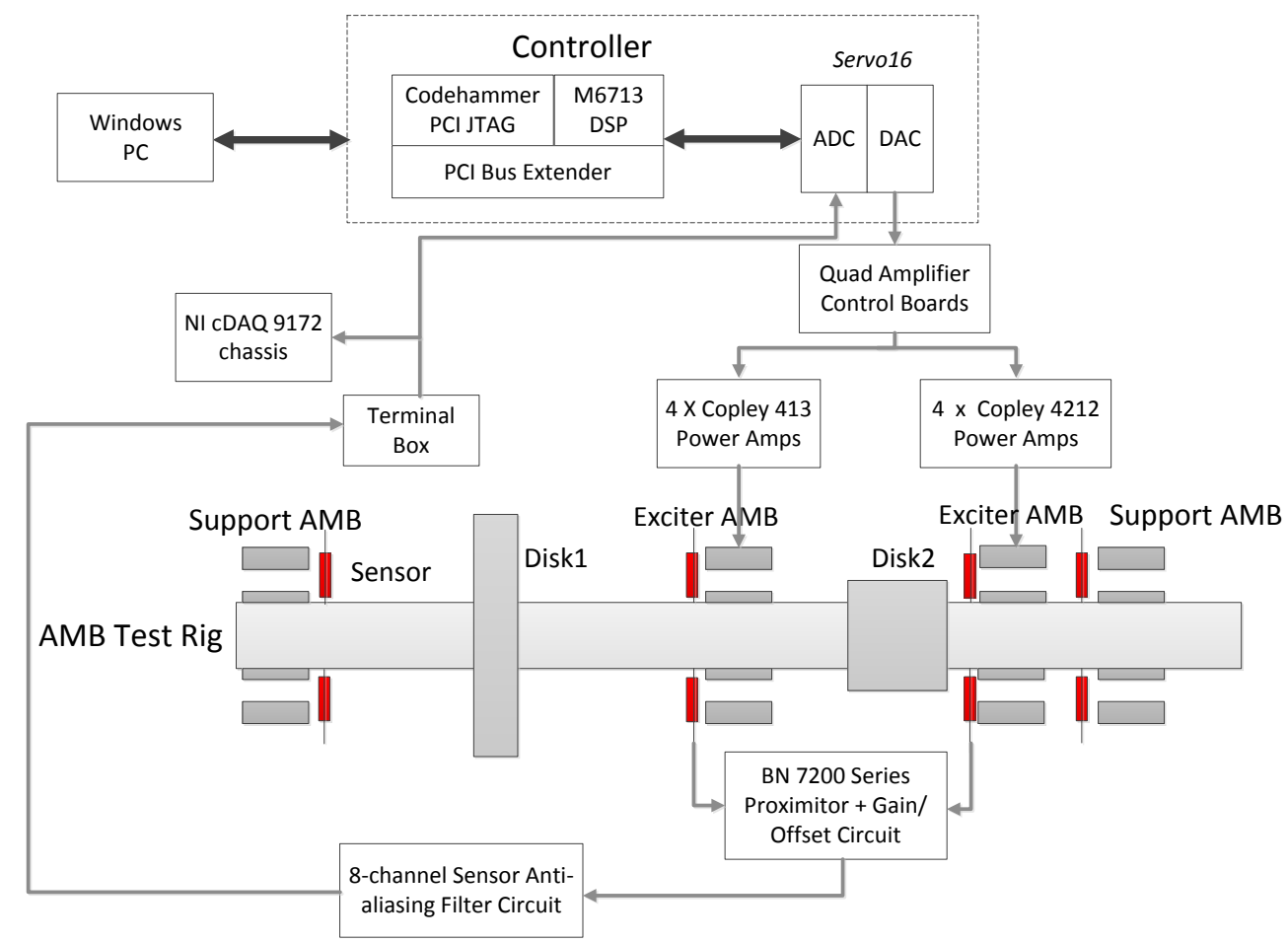

Figure 3.4: A functional overview of the exciter AMBs. 


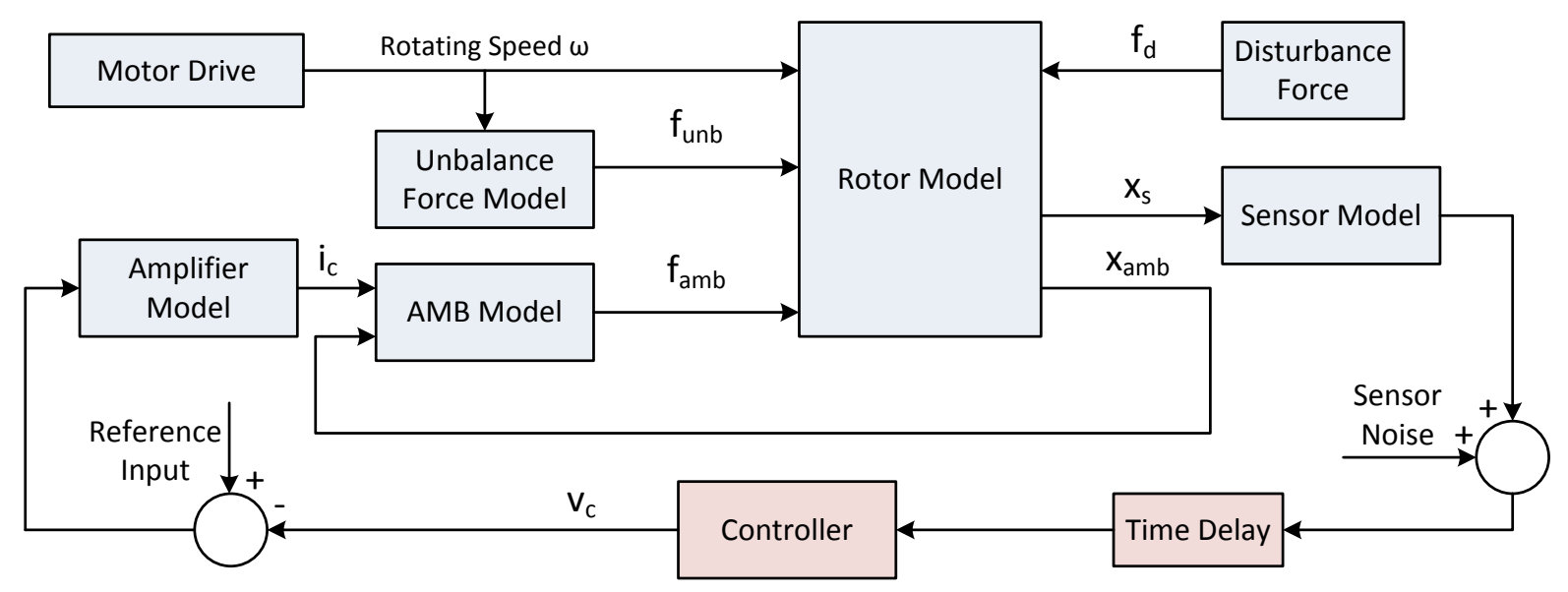

Figure 3.5: AMB system block diagram.

\subsection{AMB system model}

The AMB system model for the test rig incorporates the rotor finite element (FE) model, the linearized AMB model, the sensor and amplifier models, and the Padé approximation model of the computational delay. The block diagram of the AMB system is shown in Fig. 3.5. The following subsections elaborate the modeling of several key components in the system. A more comprehensive review of the AMB system modeling can be found in [1].

\subsubsection{Rotor model}

By dividing the rotor length into 50 stations, a two dimensional FE model of the rotor is obtained for the lateral rotordynamic analysis. A drawing of the finite element rotor model is shown in Fig. 3.6. The rotor is modeled as a lumped mass-stiffness element while the discs and the AMB laminations are modeled as mass-inertia elements added to appropriate locations. A four degree of freedom (DOF) lateral analysis is performed for each node and a ROMAC custom code is used to generate the global entries for the mass $(M)$, internal shaft stiffness $(K)$, internal shaft damping $(D)$ and gyroscopic $(G)$ matrices. Matrices $M, K$ and $D$ are symmetric and positive definite while matrix $G$ is skew-symmetric. The dynamic 


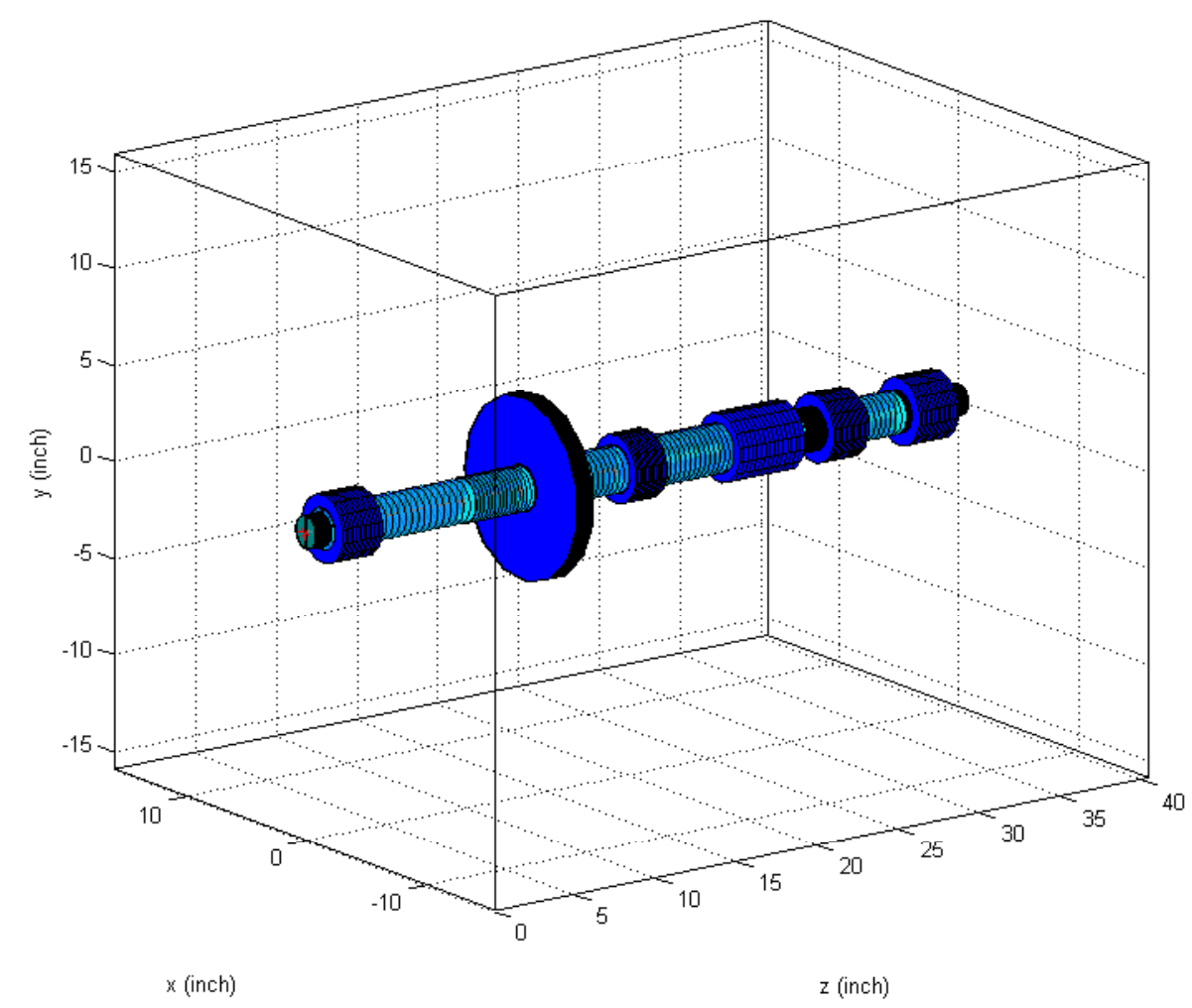

Figure 3.6: The flexible rotor finite element model.

motion of the rotor can be described in the following differential equation,

$$
\begin{aligned}
M \ddot{q}+(D+\Omega G) \dot{q}+K q & =B_{\mathrm{mag}} F_{\mathrm{mag}}+B_{w} F_{w} \\
y_{r} & =C q
\end{aligned}
$$

where the displacement vector $q$ contains 200 elements representing the lateral translations in the $x$ and $y$ axes and rotating angles around the $y$ and $x$ axes, $F_{\text {mag }}$ represents the forces provided by the AMBs and $B_{\text {mag }}$ specifies the location where the forces are injected, $F_{w}$ represents all external forces acting on the rotor with $B_{w}$ specifying the locations, $\Omega$ is the rotational speed, and the vector $y_{r}$ represents the rotor displacement at the sensor locations specified by the output matrix $C$. The modeling assumes that there is no axial motion of the rotor.

Based on the coordinate transformation between the physical space [5] and the modal 


\begin{tabular}{|l|c|c|}
\hline Mode & Free-Free & $2.5 \mathrm{MN} / \mathrm{m}$ Support Stiffness \\
\hline Nc1 & 0 & $52.2 \mathrm{~Hz}(3,130 \mathrm{rpm})$ \\
\hline Nc2 & 0 & $88.8 \mathrm{~Hz}(5,327 \mathrm{rpm})$ \\
\hline Nc3 & $224 \mathrm{~Hz}(13,433 \mathrm{rpm})$ & $243 \mathrm{~Hz}(14,596 \mathrm{rpm})$ \\
\hline Nc4 & $549 \mathrm{~Hz}(32,915 \mathrm{rpm})$ & $552 \mathrm{~Hz}(33,110 \mathrm{rpm})$ \\
\hline Nc5 & $982 \mathrm{~Hz}(58,920 \mathrm{rpm})$ & $982 \mathrm{~Hz}(58,920 \mathrm{rpm})$ \\
\hline
\end{tabular}

Table 3.2: The rotor natural frequencies.

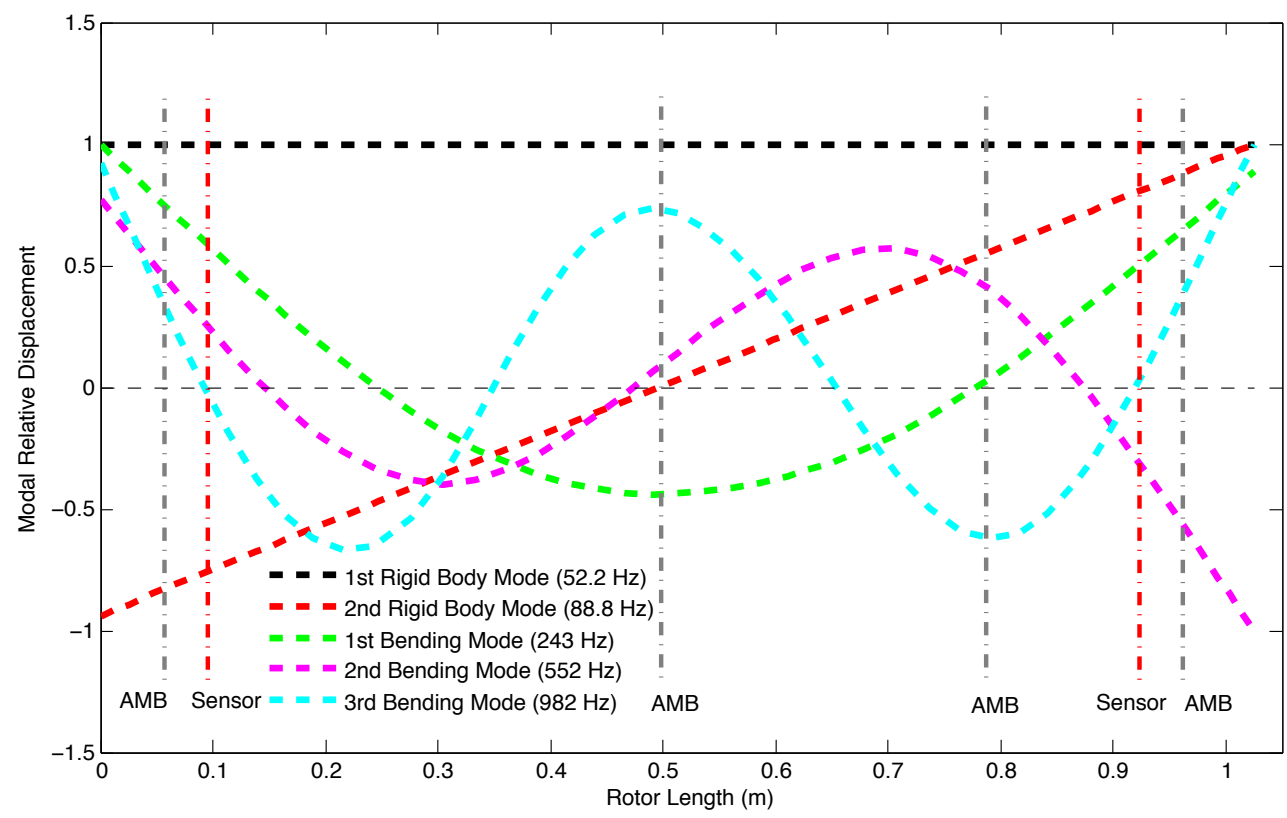

Figure 3.7: The mode shape plot of the rotor.

space using $q=\Phi_{m} \zeta$, the state space model based on Eqs. (3.1a) and (3.1b) can be converted into the following modally reduced state space form

$$
\begin{aligned}
{\left[\begin{array}{c}
\dot{\zeta}_{x}(t) \\
\dot{\zeta}_{y}(t)
\end{array}\right] } & =\left[\begin{array}{cc}
A_{\zeta} & \Omega G \\
-\Omega G & A_{\zeta}
\end{array}\right]\left[\begin{array}{l}
\zeta_{x}(t) \\
\zeta_{y}(t)
\end{array}\right]+\left[\begin{array}{cc}
\Phi_{m}^{\mathrm{T}} B_{\mathrm{mag}} & 0 \\
0 & \Phi_{m}^{\mathrm{T}} B_{\mathrm{mag}}
\end{array}\right] F_{\mathrm{mag}}+\left[\begin{array}{cc}
\Phi_{m}^{\mathrm{T}} B_{w} & 0 \\
0 & \Phi_{m}^{\mathrm{T}} B_{w}
\end{array}\right] F_{w}, \\
y_{r} & =\left[\begin{array}{cc}
C_{x} \Phi_{m} & 0 \\
0 & C_{x} \Phi_{m}
\end{array}\right]\left[\begin{array}{c}
\zeta_{x}(t) \\
\zeta_{y}(t)
\end{array}\right] .
\end{aligned}
$$

The original state space model has 400 states and contains several high order rotor modes 
beyond the controller bandwidth. These modes are unlikely to be excited and contribute negligible effect to the system dynamics. Thus, in order to facilitate analysis and design, model truncation is applied to obtain a reduced order model. The model truncation is justified by verifying the relative magnitude of the Hankel singular values of the rotor model. For the first five rotor modes, the Hankel singular values are 10.1, 10.1, 9.35, 9.32 and around 0.1 while the remaining higher order modes have significantly lower magnitudes, which verifies the higher order modes can be truncated. The mode shape plot corresponding to the first five natural frequencies of the rotor is shown in Fig. 3.7. The Campbell diagram under the original gyroscopic effects with $5 \mathrm{MN} / \mathrm{m}$ support stiffness is shown in Fig. 3.8, and it is observed that the rotor natural frequencies start dividing into forward and backward modes with the increasing of the rotational speed. The first five natural frequencies under free-free and $2.5 \mathrm{MN} / \mathrm{m}$ support stiffness are shown in Table 3.2. The final rotor model retains the two rigid body modes and the first three bending modes with a total of 20 states and is represented as follows,

$$
\begin{aligned}
\dot{x}_{m} & =A_{m} x_{m}+B_{m} F_{\mathrm{mag}}+B_{d} F_{w} \\
y_{r} & =C_{m} x_{m} .
\end{aligned}
$$

\subsubsection{Modeling of AMBs}

Here a one DOF AMB model is used to illustrate the electromagnetic force characteristics. As shown in Fig. 3.9, the forces generated by AMBs depend on the air gap between the rotor and the stator, as well as the current feedings in the windings on the stator. The following nonlinear equation is derived to describe the net force generated by a pair of electromagnets based on the movement $x$ and the air gap $g_{0}$

$$
f=f_{1}-f_{2}=\frac{\mu_{0} N^{2} A_{g} I_{1}^{2}}{4\left(g_{0}-x\right)^{2}}-\frac{\mu_{0} N^{2} A_{g} I_{2}^{2}}{4\left(g_{0}+x\right)^{2}}=\frac{\mu_{0} N^{2} A_{g}}{4}\left[\frac{I_{1}^{2}}{\left(g_{0}-x\right)^{2}}-\frac{I_{2}^{2}}{\left(g_{0}+x\right)^{2}}\right]
$$




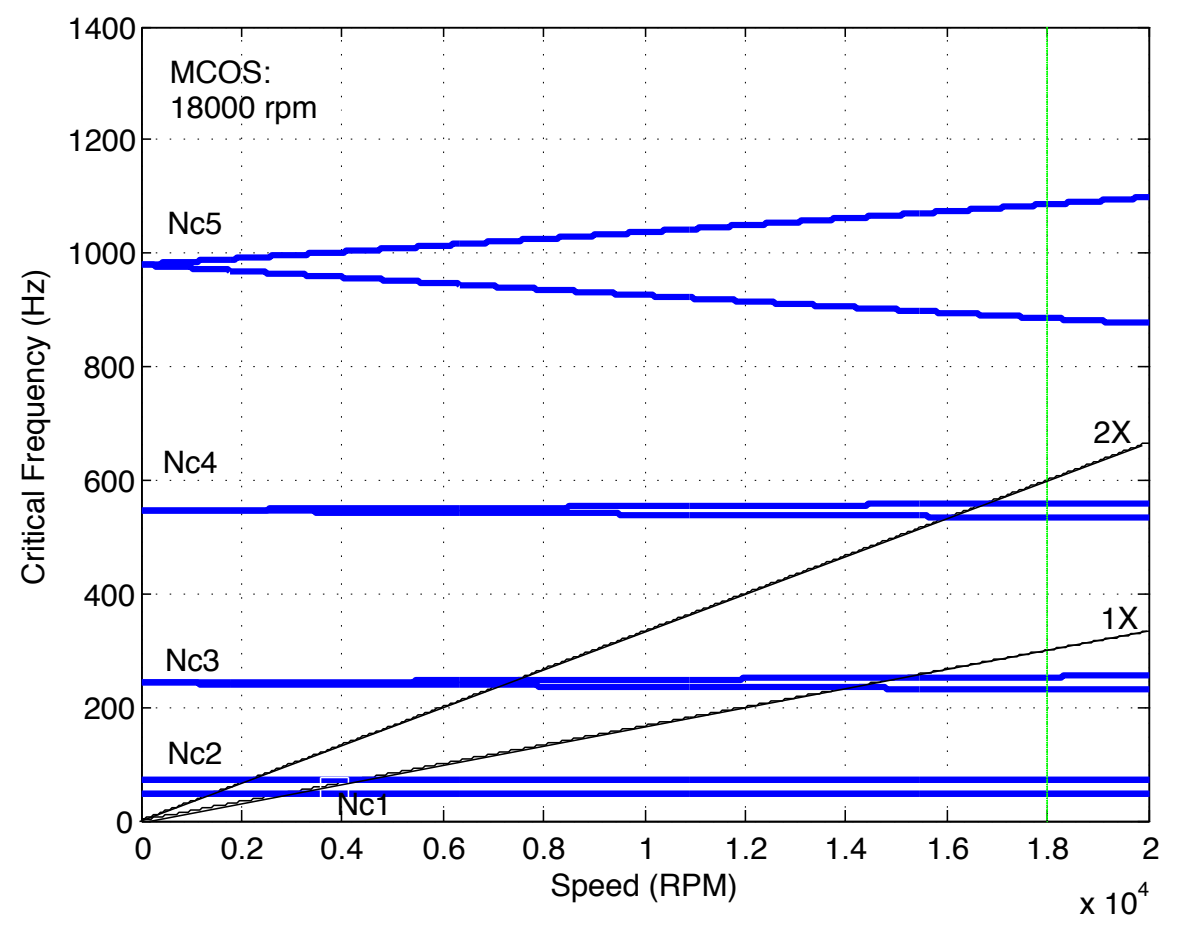

Figure 3.8: The Campbell diagram of the rotor with $5 \mathrm{MN} / \mathrm{m}$ support stiffness to show the splitting of the natural frequencies with the speed increase.

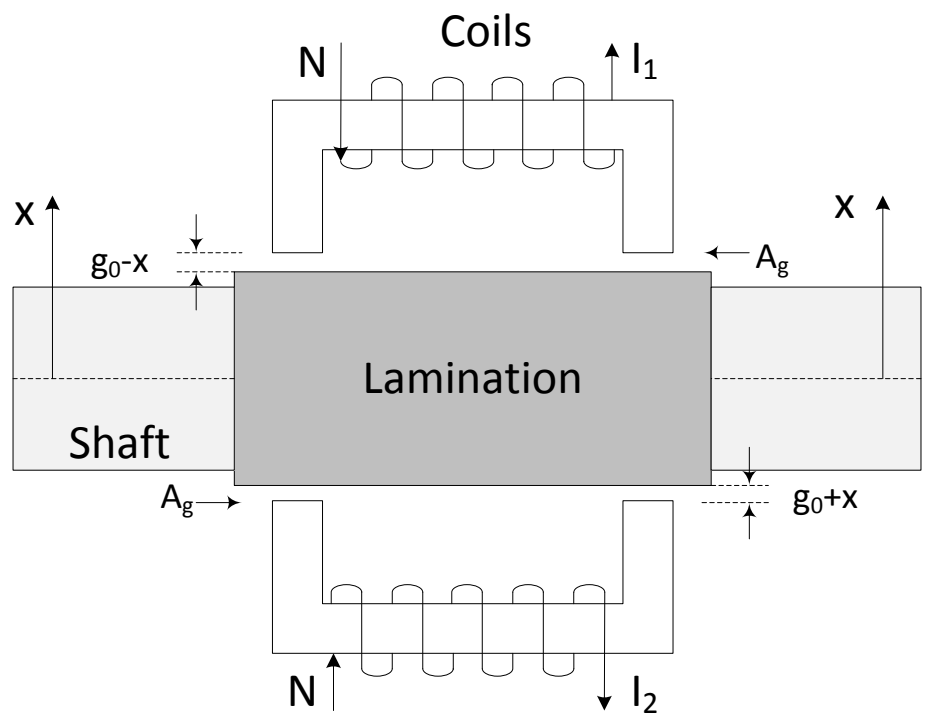

Figure 3.9: One DOF AMB model.

where $\mu_{0}$ is the permeability, $I$ is the current, $N$ is the number of turns of wires, $A_{g}$ is the face area of the pole and $g_{0}$ is the size of the air gap. 
With $I_{1}$ and $I_{2}$ being the bias current $I_{b}$ perturbed by the perturbation current $i$ as $I_{b}+i$ and $I_{b}-i$, respectively, linearization of $f$ around the equilibrium point $(i=0$ and $x=0)$ results in

$$
\begin{aligned}
& f=\frac{\mu_{0} N^{2} A_{g}}{g_{0}^{4}}\left(I_{b}^{2} g_{0} x+I_{b} i g_{0}^{2}\right)=\frac{\mu_{0} N^{2} A_{g} I_{b}^{2}}{g_{0}^{3}} x+\frac{\mu_{0} N^{2} A_{g} I_{b}}{g_{0}^{2}} i \\
& f=k_{x} x+k_{i} i
\end{aligned}
$$

where $k_{x}=\frac{\mu_{0} N^{2} A_{g} I_{b}^{2}}{g_{0}^{3}}$ and $k_{i}=\frac{\mu_{0} N^{2} A_{g} I_{b}}{g_{0}^{2}}$ are the stiffness and current gains, respectively.

This AMB model ignores the eddy current loss and assumes zero leakage in the magnetic circuit. The current gains $k_{i}$ are 130 and $100 \mathrm{~N} / \mathrm{A}$ for NDE and DE AMBs while the negative stiffness gains $k_{x}$ are 900 and $600 \mathrm{~N} / \mathrm{mm}$ for the NDE and DE AMBs with a bias current $I_{b}=4 \mathrm{~A}$. The AMB forces vector acting on the rotor in the $x$ and $y$ axes at the support

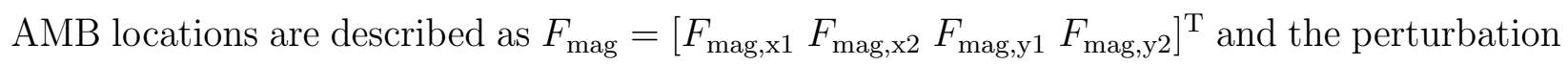
current vector is described as $i=\left[\begin{array}{llll}i_{x, 1} & i_{x, 2} & i_{y, 1} & i_{y, 2}\end{array}\right]^{\mathrm{T}}$.

Combining the rotor model with the linearized AMB model, we arrive at the following system model

$$
\begin{aligned}
\dot{x}_{m} & =\left(A_{m}-B_{m} k_{x}\left[\begin{array}{ll}
B_{\mathrm{mag}} & 0
\end{array}\right]\right) x_{m}+B_{m} k_{i} i+B_{d} F_{w}, \\
& =\hat{A}_{m} x_{m}+B_{m} k_{i} i+B_{d} F_{w}, \\
y_{r} & =C_{m} x_{m} .
\end{aligned}
$$

\subsubsection{Modeling of additional components}

The power amplifier dynamics are associated with the resistance and inductance of AMB windings as well as back-EMF and eddy current effect. Through the lamination between the rotor and the stator, the eddy current effect is reduced significantly and can be ignored in radial AMBs [34]. Based on the fitting with the experimental frequency response, the power 
amplifier is modeled by the following transfer function,

$$
G_{a}(s)=\frac{\text { DC gain }}{\left(s+p_{a 1}\right)\left(s+p_{a 2}\right)\left(s^{2}+2 \xi_{a} \omega_{a} s+\omega_{a}^{2}\right)},
$$

where the DC gain is $2.5 \mathrm{~A} / \mathrm{V}, p_{a 1}=13820 \mathrm{rad} / \mathrm{s}, p_{a 2}=28270 \mathrm{rad} / \mathrm{s}, \xi_{a}=0.55$ and $\omega_{a}=16210 \mathrm{rad} / \mathrm{s}$ with a bandwidth of $2.5 \mathrm{KHz}$ for the Copley 422 amplifiers that power the support AMBs. The amplifier transfer function $G_{a}(s)$ is then converted into a state space model $\left(A_{a}, B_{a}, C_{a}\right)$ with a total of 16 states for all the four control channels. The control voltage $u$ serves as the input to the amplifier whose output is the perturbation current $i$. Then the previous system model (3.5) can be expanded as

$$
\begin{aligned}
{\left[\begin{array}{c}
\dot{x}_{m} \\
\dot{x}_{a}
\end{array}\right] } & =\left[\begin{array}{cc}
\hat{A}_{m} & B_{m} k_{i} C_{a} \\
0 & A_{a}
\end{array}\right]\left[\begin{array}{l}
x_{m} \\
x_{a}
\end{array}\right]+\left[\begin{array}{c}
0 \\
B_{a}
\end{array}\right] u+\left[\begin{array}{c}
B_{d} \\
0
\end{array}\right] F_{w}, \\
y_{r} & =C_{m} x_{m} .
\end{aligned}
$$

For each AMB, there are four eddy current sensor probes, each of which is modeled by a constant gain $8 \mathrm{mV} / \mu \mathrm{m}$ and one analog signal conditioning circuit is also included, which serves as the anti-aliasing filter (AAF). The AAF is an 8th order Chebychev Type II low pass filter that achieves $-30 \mathrm{~dB}$ stop band attenuation at $4 \mathrm{KHz}$. The eddy current sensor and AAF are represented by a state-space model $\left(A_{s}, B_{s}, C_{s}\right)$ with 32 states and 4 voltage outputs $y_{s}$. The previous system model (3.7) can be further augmented as

$$
\begin{aligned}
{\left[\begin{array}{c}
\dot{x}_{m} \\
\dot{x}_{s} \\
\dot{x}_{a}
\end{array}\right] } & =\left[\begin{array}{ccc}
\hat{A}_{m} & 0 & B_{m} k_{i} C_{a} \\
B_{s} C_{m} & A_{s} & 0 \\
0 & 0 & A_{a}
\end{array}\right]\left[\begin{array}{c}
x_{m} \\
x_{s} \\
x_{a}
\end{array}\right]+\left[\begin{array}{c}
0 \\
0 \\
B_{a}
\end{array}\right] u+\left[\begin{array}{c}
B_{d} \\
0 \\
0
\end{array}\right] F_{w}, \\
y_{s} & =C_{s} x_{s} .
\end{aligned}
$$

There are two sampling filters at the analog front-end of the DSP board, which have flat 
magnitude response over the bandwidth of the flexible rotor AMB system and introduce a phase lag of less than $5^{\circ}$ at $1 \mathrm{KHz}$. Besides, the computational delay between each iteration is manifested as a phase lag in the frequency domain, to model the computational delay caused by the DSP. A second order Padé approximation is used to obtain a rational transfer function for a approximated time delay as follows,

$$
e^{-\tau s}=\frac{e^{-\frac{1}{2} \tau s}}{e^{\frac{1}{2} \tau s}} \approx \frac{1-\frac{1}{2} \tau s+\left(\frac{1}{2} \tau s\right)^{2}}{1+\frac{1}{2} \tau s+\left(\frac{1}{2} \tau s\right)^{2}} .
$$

The computational delay introduces a phase lag of $23^{\circ}$ at $1 \mathrm{KHz}$, which has to be taken into account in the final system model. The sampling filters from the ADC and the Padé approximation of computational delay can be formulated into a state space model $\left(A_{f}, B_{f}\right.$, $C_{f}$ ) that contributes 24 additional states.

\subsubsection{The entire AMB system}

The final system model incorporating the rotor, AMBs, amplifiers, sensors, filters and time delay can be described in the following form,

$$
\begin{aligned}
{\left[\begin{array}{c}
\dot{x}_{m} \\
\dot{x}_{s} \\
\dot{x}_{a} \\
\dot{x}_{f}
\end{array}\right]=\left[\begin{array}{cccc}
\hat{A}_{m} & 0 & B_{m} k_{i} C_{a} & 0 \\
B_{s} C_{m} & A_{s} & 0 & 0 \\
0 & 0 & A_{a} & 0 \\
0 & B_{f} C_{s} & 0 & A_{f}
\end{array}\right]\left[\begin{array}{c}
x_{m} \\
x_{s} \\
x_{a} \\
x_{f}
\end{array}\right]+\left[\begin{array}{c}
0 \\
0 \\
B_{a} \\
0
\end{array}\right] u+\left[\begin{array}{c}
B_{d} \\
0 \\
0 \\
0
\end{array}\right] F_{w} } \\
y_{f}=C_{f} x_{f} .
\end{aligned}
$$

The entire AMB system model has 92 states, which is a relatively large number for the controller synthesis. After representing all electrical/electronic components as gain plus time delay using a fourth-order Padé approximation, the following state space model $\left(A_{\mathrm{gd}}, B_{\mathrm{gd}}\right.$, 
$\left.C_{\mathrm{gd}}, D_{g d}\right)$ is derived with 16 states

$$
\begin{array}{r}
\dot{x}_{\mathrm{gd}}=A_{\mathrm{gd}} x_{\mathrm{gd}}+B_{\mathrm{gd}} y_{r}, \\
y=C_{\mathrm{gd}} x_{\mathrm{gd}}+D_{\mathrm{gd}} y_{r} .
\end{array}
$$

The gain plus delay model closely matches the magnitude and phase response of the combined model from the amplifiers, sensors, filters and approximations of computational delay. It takes the output from the rotor AMB model, which are the displacements at the sensor locations, and generates the perturbation current input to the rotor AMB model. Then the following reduced order model can be acquired at with 36 states.

$$
\begin{aligned}
{\left[\begin{array}{c}
\dot{x}_{m} \\
\dot{x}_{d}
\end{array}\right] } & =\left[\begin{array}{cc}
\hat{A}_{m} & 0 \\
B_{d} C_{m} & A_{d}
\end{array}\right]\left[\begin{array}{l}
x_{m} \\
x_{d}
\end{array}\right]+\left[\begin{array}{c}
\hat{B}_{m} \\
0
\end{array}\right] u \\
y_{s} & =\left[\begin{array}{ll}
D_{d} C_{m} & C_{d}
\end{array}\right]\left[\begin{array}{l}
x_{m} \\
x_{d}
\end{array}\right] .
\end{aligned}
$$

To be utilized for the control synthesis, the reduced order nominal plant model (3.12) is compared with experimental frequency response as shown in Fig. 3.10. Closed-loop identification is performed using a lightly tuned PID controller with filters to first levitate the rotor. Then a sinusoidal sweep from $10 \mathrm{~Hz}$ to $1200 \mathrm{~Hz}$ with fixed amplitude is generated from a spectrum analyzer and added on the controller output signal. After collecting the signal from the AAF, a frequency response is produced through the spectrum analyzer. Afterwards, similar measurements are collected from other control channels. As shown in the comparisons in Fig. 3.10, the close agreements in the frequency response among different channels indicate that the MIMO plant model captures accurate dynamics of the actual AMB system. 


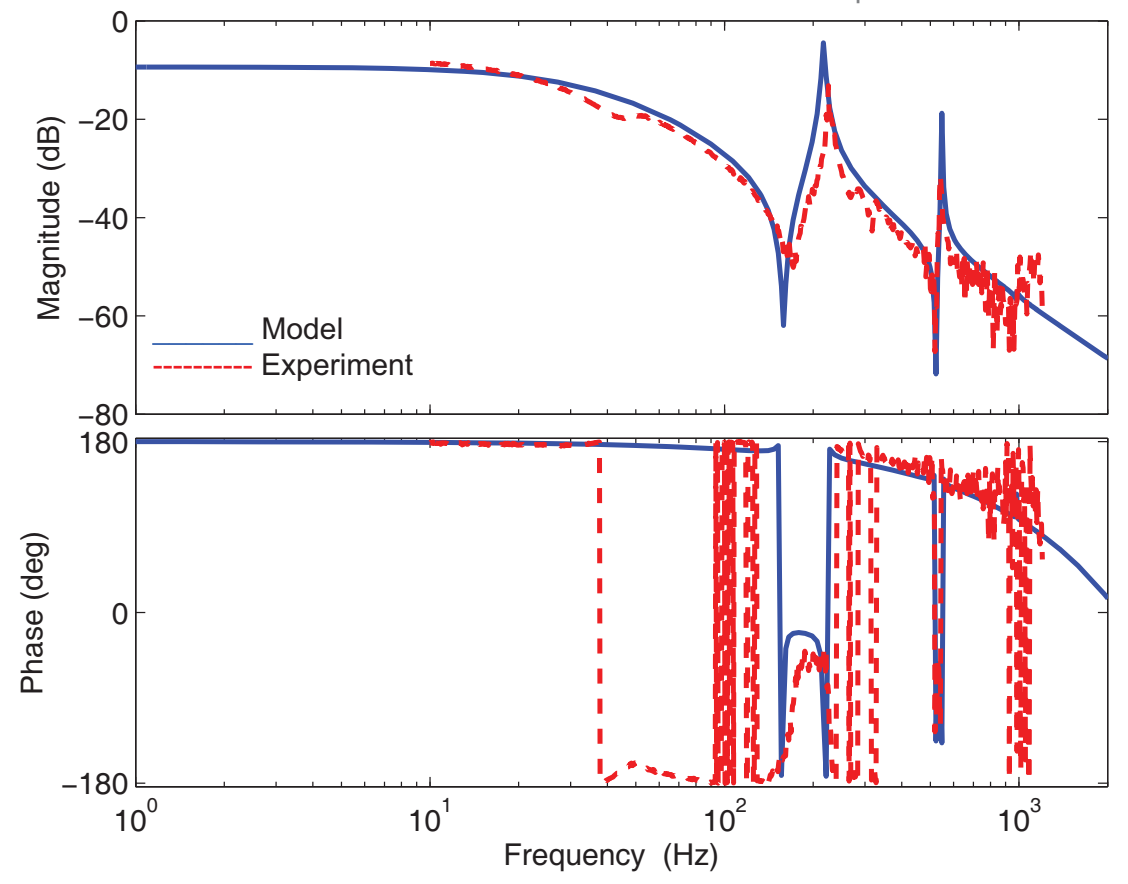

(a) NDEX-Vc to NDEX-AAFOutput



(b) DEX-Vc to NDEX-AAFOutput

Figure 3.10: Bode plots for the test rig: analytic model vs experimental measurements. 


\subsection{Summary}

This chapter first introduces the ROMAC flexible rotor AMB test rig. The overall system and the key components are described in detail. Afterwards, modeling of the AMB system is presented. The rotor FE model, the linearized magnetic circuit model for AMBs, amplifier and sensor models, and approximation of the computational time delay model are presented. The formulation of the entire AMB system model by cascading those individual components is also detailed. 


\section{Chapter 4}

\section{Fundamentals}

In this chapter, the fundamentals of controlling systems with input delay and the problem of output regulation for input-delay systems with time-invariant and time-varying exosystems are presented. First of all, the general problem formulation of a linear time-invariant (LTI) system with input delay is described and the truncated predictor feedback (TPF) control law is introduced. Then the stability condition for the input-delay system under the TPF law is developed. Afterwards, the output regulation problem for a general LTI system with input delay and time-invariant exosystem is formulated and the solution by using both state and output feedbacks are detailed. Finally, the output regulation problem is extended to a general input-delay LTI system with a time-varying exosystem, and a differential regulator based compensator design is developed to locally minimize the residual error in the regulated output for non-minimum phase systems.

\subsection{Control of systems with input delay}

Consider an LTI system with input delay, represented by the following state space equation,

$$
\dot{x}=A x+B u(\phi(t)),
$$


where $x \in \mathbf{R}^{n}$ is the state, $u \in \mathbf{R}^{m}$ is the input, $(A, B)$ is controllable and $\phi(t)$ is a known time-varying delay function defined as

$$
\phi(t)=t-D(t)
$$

where the delay function $D(t)$ satisfies that there is a $\bar{D}>0$ such that $0 \leq D(t) \leq \bar{D}$. The function $\phi(t)$ is also assumed to be continuously differentiable, invertible and $\frac{d \phi(t)}{d t}>0$ for all $t>0$.

The predictor feedback control method depends on the closed form solution of the dynamic system to predict the future system state, and the control input can be pre-computed to annul the effects of an input delay. For a constant delay $D(t)=\tau$, the future state is predicted as follows,

$$
x(t+\tau)=e^{A \tau} x(t)+\int_{t-\tau}^{t} e^{A(t-s)} B u(s) d s .
$$

Then the predictor state feedback law takes the following form

$$
u(t)=K x(t+\tau)=K e^{A \tau} x(t)+K \int_{t-\tau}^{t} e^{A(t-s)} B u(s) d s
$$

A drawback of the predictor feedback control is that the controller is infinite dimensional due to the distributed term and it is difficult to implement in practice.

A stabilizing controller for Eq. (4.1) could be constructed through the finite dimensional predictor feedback control method as discussed in [3], which is also known as the truncated predictor feedback (TPF) method. For a constant delay, the TPF control law with a feedback gain $K$ is given as

$$
u(t)=K e^{A\left(\phi^{-1}(t)-t\right)} x(t)=K e^{A \tau} x(t) .
$$

The state space equation of the closed-loop system (4.1) under the TPF control can be found as

$$
\dot{x}(t)=(A+B K) x(t)-B K \lambda(t)
$$


where $\lambda(t)$ is defined as

$$
\begin{aligned}
\lambda(t) & =\int_{\phi(t)}^{t} e^{A(t-s)} B K e^{A(s-\phi(s))} x(\phi(s)) \mathrm{d} s, \\
& =\int_{t-\tau}^{t} e^{A(t-s)} B K e^{A \tau} x(s) \mathrm{d} s .
\end{aligned}
$$

The feedback gain $K$ that stabilizes the closed-loop system can be obtained from the following theorem [41]:

Theorem 4.1.1 Consider the input-delay system (4.1), where the pair $(A, B)$ is controllable. Given some positive definite matrix $W$, the TPF control (4.6) with

$$
K=-B^{\mathrm{T}} W^{-1}
$$

asymptotically stabilizes the delayed system (4.1) if there exist real scalars $\omega>0$ and $\alpha>0$ such that

$$
\begin{aligned}
& \alpha W \geq B B^{\mathrm{T}}, \\
&\left(A-\frac{1}{2} \omega I\right) W+W\left(A-\frac{1}{2} \omega I\right)^{\mathrm{T}}<0, \\
& W\left(A+\frac{\alpha^{3} \bar{D}}{2 \omega} e^{\omega \bar{D}}\left(e^{\omega \bar{D}}-1\right) I\right)^{\mathrm{T}} \\
&+\left(A+\frac{\alpha^{3} \bar{D}}{2 \omega} e^{\omega \bar{D}}\left(e^{\omega \bar{D}}-1\right) I\right) W<B B^{\mathrm{T}} .
\end{aligned}
$$

The above theorem provides the stability conditions for the input-delay system under the TPF control law. These conditions have been established from the solution to an algebraic Riccati equation, and the details are shown in [41]. Based on Theorem 4.1.1, the optimal TPF control law that maximizes the allowable delay bound $\bar{D}$ for the stable closed-loop system can be obtained. The process of deriving the controller with the maximized delay bound will be detailed in the next chapter. 


\subsection{Output regulation for input-delay system with time- invariant exosystem}

Output regulation is one of the central problems in control theory and the objective is to regulate the plant output so that it tracks a prescribed reference signal in the presence of disturbances. The reference and disturbance signals perturbing the system are produced by an external dynamic system known as the exosystem. The output regulation problem has been extensively studied by many researchers since it was formulated by [42] for linear systems, and by [43] for nonlinear systems. A comprehensive treatment of the nonlinear output regulation problem can be found in [44, 45].

Several works have studied the output regulation problem for time delay systems. The output regulation problem for systems with state delays has been discussed in [46] and [47], where the authors derive the necessary and sufficient conditions for the existence of a solution by employing an argument similar to one as presented in [42] for the delay-free case. In the nonlinear system setting, [48] extends the solvability conditions of the output regulation problem to systems with state delays. For systems with input delay, [49] introduces a robust solution to the output regulation problem. For continuous time linear time-invariant system with delays in the state, input and output, the output regulation problem has been studied in $[50,51]$. Few works that apply the output regulation problem with time delays to practical systems can be found in the literature.

The exosystem studied in this section is assumed to be time-invariant and is extended to time-varying exosystem in Section 4.3.

Consider the following LTI system with input delay,

$$
\begin{aligned}
\dot{x}(t) & =A x(t)+B u(t-\tau)+P w(t), \\
y(t) & =G x(t), \\
e(t) & =C x(t)+D u(t-\tau)+Q w(t),
\end{aligned}
$$


where state $x \in \mathbf{R}^{n}$, control input $u \in \mathbf{R}^{m}$, output $y \in \mathbf{R}^{r}$, error to be regulated $e \in \mathbf{R}^{r}$ and $\tau \geq 0$

The external disturbance $w$ is generated by the following linear exosystem

$$
\dot{w}(t)=S w(t)
$$

In general, $w$ could be of any dimension. The objective for the output regulation problem is to find a control law such that

1. The closed-loop system is asymptotically stable when $w \equiv 0$.

2. The regulation error $e(t)$ approaches zero as $t \rightarrow \infty$, for any arbitrary initial conditions of $x$ and $w$.

Some standard assumptions are made on the system (4.10) that are required for the solvability of the output regulation problem.

Assumption 1 The system (4.10) is stabilizable and detectable when $w \equiv 0$.

Assumption 2 The eigenvalues of the exosystem (4.11) have nonnegative real parts.

Assumption 1 guarantees that there exists a control law using the output information available to asymptotically stabilize system (4.10). Assumption 2 ensures that the output regulation problem is nontrivial and the disturbance signal generated by the exosystem will not converge to zero.

\subsubsection{Output regulation by state feedback}

The following lemma provides a solution to the output regulator problem by state feedback, given that a stabilizing control law exists for the disturbance-free system. 
Lemma 4.2.1 Consider the time-delay system (4.10) satisfying Assumptions 1 and 2 , and let the state feedback law $u(t)=\kappa x(t), \kappa \in \mathbf{R}^{m \times n}$, be an asymptotically stabilizing control law for (4.10) when $w \equiv 0$. Then, the control law

$$
u(t)=\nu(t)+\Gamma e^{\tau S} w(t)
$$

where $\nu(t)=\kappa z(t)$ and $z(t)=x(t)-\Pi w(t)$, solves the output regulation problem if and only if there exist matrices $\Pi \in \mathbf{R}^{n \times 2 s}$ and $\Gamma \in \mathbf{R}^{m \times 2 s}$ that satisfy the regulator equations

$$
\begin{array}{r}
\Pi S=A \Pi+B \Gamma+P, \\
0=C \Pi+D \Gamma+Q .
\end{array}
$$

Proof. The proof follows readily from defining the state transformation $z(t)=x(t)-\Pi w(t)$, and the control input to be in the form of (4.12). Let $\Pi$ and $\Gamma$ be the solution to (4.13). Then (4.10) simplifies to

$$
\begin{aligned}
& \dot{z}(t)=A z(t)+B \nu(t-\tau), \\
& e(t)=C z(t)+D \nu(t-\tau),
\end{aligned}
$$

which is equivalent to (4.10) with $w \equiv 0$. Assumption 1 implies that there exists a stabilizing state-feedback control law $\nu(t)=\kappa z(t)$, and the fact that $\lim _{t \rightarrow \infty} e(t)=0$ leads to the conclusion that (4.12) is a solution to the output regulation problem.

To demonstrate the necessary condition, assume that (4.12) is a solution to the output regulation problem. Then, the regulation error becomes

$$
e(t)=C z(t)+D \kappa z(t-\tau)+(C \Pi+D \Gamma+Q) w(t)
$$


which must satisfy that $\lim _{t \rightarrow \infty} e(t)=0$. Since Assumption 2 states that $w(t)$ is nonvanishing, the regulation error approaches zero for arbitrary initial conditions if $\lim _{t \rightarrow \infty} z(t)=0$ and (4.13b) is true. Furthermore, it was assumed that $\nu(t)=\kappa z(t)$ asymptotically stabilizes (4.14). For $\lim _{t \rightarrow \infty} z(t)=0$ to be true under Assumption 2, it is required that (4.13a) is satisfied.

For the undisturbed system (4.10) with $w \equiv 0$, the TPF control takes the form of a finite dimensional predictor feedback law as discussed in Section 4.1 as follows,

$$
u(t)=K e^{A \tau} x(t)
$$

where $K$ is the feedback gain with $K=-B^{\mathrm{T}} W^{-1}$ and $W$ is derived based on the stability conditions described in (4.9).

\subsubsection{Output regulation by output feedback}

For practical implementation purposes, the state feedback results are extended to the output feedback design.

Consider Eqs. (4.10) and (4.11) in the following state space form with $\chi=\left[x^{\mathrm{T}} w^{\mathrm{T}}\right]^{\mathrm{T}}$,

$$
\begin{aligned}
& \dot{\chi}(t)=\left[\begin{array}{ll}
A & P \\
0 & S
\end{array}\right] \chi(t)+\left[\begin{array}{l}
B \\
0
\end{array}\right] u(t-\tau), \\
& y(t)=\left[\begin{array}{ll}
G & 0
\end{array}\right] \chi(t), \\
& e(t)=\left[\begin{array}{ll}
C & Q
\end{array}\right] \chi(t)+D u(t-\tau) .
\end{aligned}
$$

Lemma 4.2.2 Consider the time-delay system (4.16) satisfying Assumptions 1 and 2 , and let the state feedback law

$$
u(t)=\kappa z(t)+\Gamma e^{\tau \mathcal{S}} w(t)
$$


as defined in Lemma 4.2.1, be a solution to the problem of output regulation by state feedback, where $z(t)=x(t)-\Pi w(t)$, and $\Pi \in \mathbf{R}^{n \times 2 s}$ and $\Gamma \in \mathbf{R}^{m \times 2 s}$ satisfy the regulator equations in (4.13). Then, the output feedback control law

$$
\begin{aligned}
& \dot{\hat{\chi}}(t)=\mathbb{A} \hat{\chi}(t)+\mathbb{B} u(t-\tau)+L(\mathbb{C} \hat{\chi}(t)-y(t)),
\end{aligned}
$$

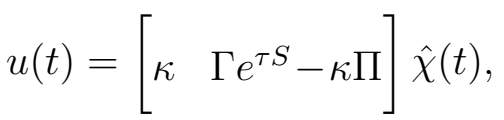

solves the output regulation problem, where

$$
\hat{\chi}=\left[\begin{array}{l}
\hat{x} \\
\hat{w}
\end{array}\right], \mathbb{A}=\left[\begin{array}{ll}
A & P \\
0 & S
\end{array}\right], \mathbb{B}=\left[\begin{array}{l}
B \\
0
\end{array}\right], \mathbb{C}=\left[\begin{array}{ll}
G & 0
\end{array}\right],
$$

if a matrix $L \in \mathbf{R}^{r \times m}$ exists such that $(\mathbb{A}+L \mathbb{C})$ is Hurwitz.

Proof. Similarly to the proof of Lemma 4.2.1, the state transformation is defined as $z(t)=x(t)-\Pi w(t)$, and let $\hat{z}=\hat{x}-\Pi \hat{w}$ and $\tilde{z}=\hat{z}-z$. The closed-loop equation of $z(t)$ and the regulation error $e(t)$ respectively become

$$
\begin{aligned}
\dot{z}(t)=A z(t)+B \kappa z(t-\tau) & \\
+ & {\left[\begin{array}{cc}
B \kappa & B\left(\Gamma e^{\tau S}-\kappa \Pi\right)
\end{array}\right] \tilde{\chi}(t-\tau), } \\
e(t)=C z(t)+D \kappa z(t-\tau) & +\left[\begin{array}{ll}
D \kappa & D\left(\Gamma e^{\tau S}-\kappa \Pi\right)
\end{array}\right] \tilde{\chi}(t-\tau),
\end{aligned}
$$

where the equation for $\tilde{\chi}=\hat{\chi}-\chi$ is obtained from (4.16) and (4.17) as

$$
\dot{\tilde{\chi}}(t)=(\mathbb{A}+L \mathbb{C}) \tilde{\chi}(t)
$$

The $z(t)$ and $\tilde{\chi}(t)$ subsystems are decoupled, and (4.19) is asymptotically stable. Lemma 4.2.1 also yields that $z(t)$ asymptotically approaches zero when $\tilde{\chi} \equiv 0$. Finally, as the 
states $z(t)$ and $\tilde{\chi}(t)$ asymptotically approach zero, the regulation error signal will also converge to zero. Therefore, (4.17) is a solution to the output regulation problem by output feedback.

\subsection{Output regulation for input-delay system with time- varying exosystem}

Most of existing literature on the output regulation problem assumes the exosystem to be time-invariant. However, for many practical systems, the external disturbance is time-varying, so it is worthwhile to investigate the output regulation problem with a time-varying exosystem. Only a few works have considered this problem [52-54] and the linear differential regulator equation becomes the essential part in tackling the time-varying exosystem. All the previous results are restricted to minimum phase systems, except [55], where the results are extended to non-minimum phase systems. Throughout the literature, systems with input delay have not been considered in the output regulation problem with a time-varying exosystem.

First consider the following LTI plant with a constant input delay

$$
\begin{aligned}
\dot{x}(t) & =A x(t)+B u(t-\tau)+P w(t), \\
y(t) & =H x(t), \\
e(t) & =C x(t)+D u(t-\tau)+Q w(t) .
\end{aligned}
$$

In (4.20), the state vector $x \in \mathbf{R}^{n}$, the control input vector $u \in \mathbf{R}^{m}$, the plant output vector $y \in \mathbf{R}^{r}$ and the error to be regulated $e \in \mathbf{R}^{r}$.

Consider the following linear time-varying exosystem

$$
\dot{w}(t)=S(t) w(t)
$$


where $w \in \mathbf{R}^{p}$ represents the disturbance to be rejected $(P w(t))$ and the reference signal to be tracked $(Q(w(t)), S(t))$ is bounded and smooth with bounded derivatives.

For convenience, a matrix $A(t)$ is said to be exponentially stable if the linear system $\dot{x}=A(t) x$ is exponentially stable, which means that the corresponding state transition matrix $\phi_{A}\left(t, t_{0}\right)$ satisfies $\left\|\phi_{A}\left(t, t_{0}\right)\right\| \leq c_{0} e^{-\alpha\left(t-t_{0}\right)}$ with $t \geq t_{0}$ for some $c_{0}>0$ and $\alpha>0$.

The objective of the output regulation is to find a control law such that

- The closed-loop system is asymptotically stable when $w \equiv 0$;

- The error $e(t)$ converges to zero, for any initial conditions of the plant and the exosystem.

Two assumptions are made for the solvability of the output regulation problem.

Assumption 3 The system (4.20) is stabilizable and detectable when $w \equiv 0$.

Assumption 4 The state transition matrix for the time-varying exosystem is uniformly bounded for all $t$ and $t_{0}$, that is, $c_{1} \leq\left\|\phi_{S}\left(t, t_{0}\right)\right\| \leq c_{2}$ for some positive constants $c_{1}$ and $c_{2}$.

Assumption 3 is required for the existence of a control law that asymptotically stabilizes the system (4.20) when $w \equiv 0$. Assumption 4 does not affect the generality of the problem but facilitates the solution of the output regulation problem.

\subsubsection{Output regulation by state feedback}

Given that a stabilizing control law exists for the disturbance-free system with input delay, the following lemma provides a solution to the output regulation problem by state feedback.

Lemma 4.3.1 Consider a time-delay system (4.20) that satisfies both Assumptions 3 and 4. The state feedback control law

$$
u(t)=\kappa x(t)+\left(R(t) K_{d}-\kappa \Pi(t)\right) w(t),
$$


where $\kappa$ is the state feedback gain that asymptotically stabilizes the system when $w \equiv 0$ and $K_{d}=\phi_{S}\left(t, t_{0}\right) \phi_{S}^{-1}\left(t-\tau, t_{0}\right)$, achieves output regulation if and only if there exist matrices $R(t) \in \mathbf{R}^{m \times a}$ and $\Pi(t) \in \mathbf{R}^{n \times a}$ and satisfy the following differential regulator equation (DRE)

$$
\begin{aligned}
& \dot{\Pi}(t)+\Pi(t) S(t)=A \Pi(t)+B R(t)+P \\
& \lim _{t \rightarrow \infty}(C \Pi(t)+D R(t)+Q)=0 .
\end{aligned}
$$

Proof. The proof starts with defining the state transformation $z(t)=x(t)-\Pi(t) w(t)$. The dynamic eqaution of $z(t)$ and the regulation error $e(t)$ can be written as

$$
\begin{aligned}
& \dot{z}(t)=A z(t)+B u(t-\tau)+A \Pi(t) w(t)+P w(t)-\Pi(t) S(t) w(t)-\dot{\Pi}(t) w(t), \\
& e(t)=C z(t)+D u(t-\tau)+C \Pi(t) w(t)+Q w(t) .
\end{aligned}
$$

Consequently, the control law (4.22) can be written as $u(t)=\kappa z(t)+R(t) K_{d} w(t)$. Let $R(t)$ and $\Pi(t)$ be solution of the DRE (4.23). The equations for $\dot{z}(t)$ and $e(t)$ then simplify to

$$
\begin{aligned}
& \dot{z}(t)=A z(t)+B \kappa z(t-\tau), \\
& e(t)=C z(t)+D \kappa z(t-\tau) .
\end{aligned}
$$

Since $\kappa$ is the state feedback gain that asymptotically stabilizes the input-delay system when $w \equiv 0, \lim _{t \rightarrow \infty} z(t)=0$ and hence $\lim _{t \rightarrow \infty} e(t)=0$. Thus, control law (4.22) solves the output regulation problem.

To demonstrate the necessary condition, assuming the state feedback control law (4.22) is a solution of the output regulation problem, then $e(t)$ becomes

$$
e(t)=C z(t)+D \kappa z(t-\tau)+(C \Pi(t)+D R(t)+Q) w(t)
$$


which must satisfy that $\lim _{t \rightarrow \infty} e(t)=0$. Since Assumption 4 states that $w(t)$ is nonvanishing, $e(t)$ approaches 0 for arbitrary initial conditions if $\lim _{t \rightarrow \infty} z(t)=0$ and $(4.23 \mathrm{~b})$ is true. Furthermore, it is assumed that under $u(t)=\kappa z(t)+R(t) K_{d} w(t),(4.24)$ are established for $z(t)$ and $e(t)$ to be asymptotically stable. For $\lim _{t \rightarrow \infty} z(t)=0$ to be true under Assumption 4, (4.23a) is required to be satisfied.

\subsubsection{Output regulation by output feedback}

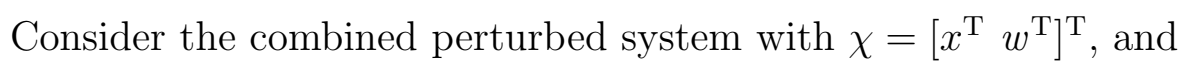

$$
\begin{aligned}
& \dot{\chi}(t)=\left[\begin{array}{cc}
A & P \\
0 & S(t)
\end{array}\right] \chi(t)+\left[\begin{array}{l}
B \\
0
\end{array}\right] u(t-\tau), \\
& y(t)=\left[\begin{array}{ll}
H & 0
\end{array}\right] \chi(t), \\
& e(t)=\left[\begin{array}{ll}
C & Q
\end{array}\right] \chi(t)+D u(t-\tau) .
\end{aligned}
$$

Lemma 4.3.2 Consider the system (4.25) satisfying both Assumptions 3 and 4, and let the following state feedback control law

$$
u(t)=\kappa x(t)+\left(R(t) K_{d}-\kappa \Pi(t)\right) w(t)
$$

be the solution to the state feedback output regulation problem from Lemma 4.3.1. Then the following output feedback control law

$$
\begin{aligned}
& \dot{\hat{\chi}}(t)=\mathbb{A}(t) \hat{\chi}(t)+\mathbb{B} u(t-\tau)+L(\mathbb{C} \hat{\chi}(t)-y(t)),
\end{aligned}
$$

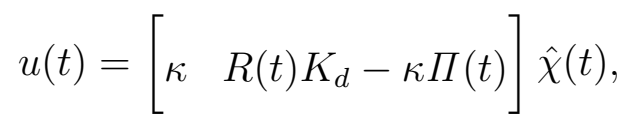


4.3 Output regulation for input-delay system with time-varying exosystem

where

$$
\hat{\chi}=\left[\begin{array}{c}
\hat{x} \\
\hat{w}
\end{array}\right], \mathbb{A}(t)=\left[\begin{array}{cc}
A & P \\
0 & S(t)
\end{array}\right], \mathbb{B}=\left[\begin{array}{l}
B \\
0
\end{array}\right], \mathbb{C}=\left[\begin{array}{ll}
H & 0
\end{array}\right]
$$

$\hat{x}$ and $\hat{w}$ are the estimated states, achieves the output regulation objectives if the transition matrix $\phi_{\Xi^{L}}$ corresponding to $\Xi^{L}(t)=\mathbb{A}(t)+L \mathbb{C}$ and $L \in \mathbf{R}^{r \times m}$ satisfies $\left\|\phi_{\Xi^{L}}\left(t, t_{0}\right)\right\| \leq$ $k_{L} e^{-l_{0}\left(t-t_{0}\right)}$ for some $k_{L}>0$ and $l_{0}>0$.

Proof. The proof starts with defining the state transformation $z(t)=x(t)-\Pi(t) w(t)$. Let $\hat{z}=\hat{x}-\Pi \hat{w}$ and $\tilde{z}=\hat{z}-z$. The closed-loop equation of the $z(t)$ and the regulation error $e(t)$ can be written as

$$
\begin{aligned}
\dot{z}(t)= & A z(t)+A \Pi(t) w(t)+P w(t)-\Pi(t) S(t) w(t)-\dot{\Pi}(t) w(t)+B \kappa \hat{z}(t-\tau) \\
& +B R(t) K_{d} \hat{w}(t-\tau), \\
e(t)= & C z(t)+C \Pi(t) w(t)+Q w(t)+D \kappa \hat{z}(t-\tau)+D R(t) K_{d} \hat{w}(t-\tau),
\end{aligned}
$$

which, in view of $\hat{z}=\tilde{z}+z$ and $\hat{w}=\tilde{w}+w$, can be written as

$$
\begin{aligned}
& \dot{z}(t)=A z(t)+B \kappa z(t-\tau)+\left[\begin{array}{cc}
B \kappa & B\left(R(t) K_{d}-\kappa \Pi(t)\right)
\end{array}\right] \tilde{\chi}(t-\tau), \\
& e(t)=C z(t)+D \kappa z(t-\tau)+\left[\begin{array}{cc}
D \kappa & D\left(R(t) K_{d}-\kappa \Pi(t)\right)
\end{array}\right] \tilde{\chi}(t-\tau),
\end{aligned}
$$

where $\tilde{\chi}(t)=\hat{\chi}(t)-\chi(t)$. The dynamics of $\tilde{\chi}(t)$ is given by

$$
\dot{\tilde{\chi}}(t)=(\mathbb{A}(t)+L \mathbb{C}) \tilde{\chi}(t) .
$$

With the assumption of $\left\|\phi_{\Xi^{L}}\left(t, t_{0}\right)\right\| \leq k_{L} e^{-l_{0}\left(t-t_{0}\right)}$ for $\Xi^{L}(t)=\mathbb{A}(t)+L \mathbb{C}$, Eq. (4.27) is asymptotically stable and as the states $z(t)$ and state estimation errors $\tilde{\chi}(t)$ asymptotically approach zeros, $\lim _{t \rightarrow \infty} e(t)=0$. 


\subsubsection{Bounded regulator gains for non-minimum phase systems}

From the previous subsection, it can be observed that the output regulation problem with time-varying exosystem is solved if bounded solutions are found to the corresponding DRE. For minimum phase systems, the solution to (4.23) is always bounded, and numerical methods can be used to construct the output regulation control law. When the system is of nonminimum phase, such as AMB systems, the solution to (4.23) may become unbounded and the applicability of the current approach is restricted to a small number of special cases [55]. In this subsection, a sub-optimal solution to the output regulation problem is proposed for non-minimum phase systems, such that the solution to the corresponding DRE is always bounded and the residual error in the regulated output is locally minimized.

A coordinate transformation is adopted to convert the system (4.20) into a normal form as described in [56]. Denote the $j$ th row of the matrix $C$ by $C_{j}$. For a set of positive integers $r_{1}, r_{2}, \ldots, r_{p}$, where $p$ is the number of outputs, the row vector $\tau_{j}^{i}$ is defined as $\tau_{1}^{i}:=C_{i}, 1 \leq i \leq p, \tau_{j+1}^{i}:=\tau_{j}^{i} A, 1 \leq j \leq r_{i}-1$. The other set of row vector $\breve{\tau}_{j}^{i}$ is defined as $\breve{\tau}_{1}^{i}:=Q_{i}, 1 \leq i \leq p$, and $\breve{\tau}_{j+1}^{i}:=\breve{\tau}_{j}^{i} S+\tau_{j}^{i} P, 1 \leq j \leq r_{i}-1$.

Assumption 5 There exist positive integers $r_{1}, r_{2}, \ldots, r_{p}$ known as the 'vector relative degree' [57] such that $r=r_{1}+r_{2}+\cdots+r_{p} \leq n, \tau_{j}^{i} B=0$ for $1 \leq i \leq p$ and $1 \leq j \leq r_{i}-1$, and the rank of the matrix $\Psi=\left[\left(\tau_{r_{1}}^{1}\right)^{\mathrm{T}}\left(\tau_{r_{2}}^{2}\right)^{\mathrm{T}} \cdots\left(\tau_{r_{p}}^{p}\right)^{\mathrm{T}}\right]^{\mathrm{T}} B \in \mathbf{R}^{p \times m}$ is $p$.

Define the matrices

$$
\begin{aligned}
& \bar{T}^{x}=\left[\begin{array}{lllll}
\tau_{1}^{0 \mathrm{~T}} \cdots & \tau_{n-r}^{0 \mathrm{~T}} & \tau_{1}^{1 \mathrm{~T}} \cdots \tau_{r_{1}}^{1 \mathrm{~T}} & \tau_{1}^{2 \mathrm{~T}} \cdots \tau_{r_{p}}^{p \mathrm{~T}}
\end{array}\right]^{\mathrm{T}}, \\
& T^{w}=\left[\begin{array}{lllll}
0 \cdots 0 & \breve{\tau}_{1}^{1 \mathrm{~T}} \cdots \breve{\tau}_{r_{1}}^{1 \mathrm{~T}} & \breve{\tau}_{1}^{2 \mathrm{~T}} \cdots \breve{\tau}_{r_{p}}^{p \mathrm{~T}}
\end{array}\right]^{\mathrm{T}},
\end{aligned}
$$

where $\tau_{1}^{0}, \tau_{2}^{0}, \ldots, \tau_{n-r}^{0}$ are vectors that make $\bar{T}^{x}$ a transformation matrix whose existence is guaranteed by Assumption 5. A $w$-dependent coordinate transfomration from $x$ to $(\bar{\beta}, \zeta)$ is given as $\left[\begin{array}{c}\bar{\beta} \\ \zeta\end{array}\right]=\bar{T}^{x} x+T^{w} w$, to generate the following multi-input multi-output (MIMO) 
normal form [57]:

$$
\begin{aligned}
& \dot{\bar{\beta}}=\bar{\Gamma} \bar{\beta}+\bar{\Lambda} \zeta+\bar{\Theta} w, \\
& \dot{\zeta}_{i-1}^{j}=\zeta_{i}^{j}, \text { for } i=2,3, \ldots, r_{j} \text { and } j=1,2, \ldots, p, \\
& {\left[\dot{\zeta}_{r_{1}}^{1}, \dot{\zeta}_{r_{2}}^{2}, \ldots, \dot{\zeta}_{r_{p}}^{p}\right]^{\mathrm{T}}=\bar{E} \bar{\beta}+M \zeta+\Psi u+N w,} \\
& e=\left[\zeta_{1}^{1}, \zeta_{1}^{2}, \ldots, \zeta_{1}^{p}\right]^{\mathrm{T}} .
\end{aligned}
$$

Assumption 6 There exists a coordinate transformation $T^{\beta}$ such that, under the coordinate change $\beta=\left[\begin{array}{c}\beta^{a} \\ \beta^{s}\end{array}\right]=T^{\beta} \bar{\beta}, \beta^{a} \in \mathbf{R}^{k}, \beta^{s} \in \mathbf{R}^{n-r-k}$, the zero dynamics $\overline{\bar{\beta}}=\bar{\Gamma} \bar{\beta}$ becomes

$$
\left[\begin{array}{c}
\dot{\beta}^{a} \\
\dot{\beta}^{s}
\end{array}\right]=\left[\begin{array}{cc}
\Gamma^{\mathrm{a}} & 0 \\
0 & \Gamma^{\mathrm{s}}
\end{array}\right]\left[\begin{array}{l}
\beta^{a} \\
\beta^{s}
\end{array}\right]=\Gamma \beta,
$$

where all eigenvalues of $\Gamma^{\mathrm{s}}$ have negative real parts and all eigenvalues of $\Gamma^{\mathrm{a}}$ have non-negative real parts.

Based on Assumptions 5 and 6, the coordinate transformation

$$
\left[\begin{array}{c}
\beta \\
\zeta
\end{array}\right]=\left[\begin{array}{cc}
T^{\beta} & 0 \\
0 & I_{r \times r}
\end{array}\right] \bar{T}^{x} x+T^{w} w=T^{x} x+T^{w} w
$$

yields the following normal form for system (4.20)

$$
\begin{aligned}
& \dot{\beta}=\Gamma \beta+\Lambda \zeta+\Theta w, \\
& \dot{\zeta}_{i-1}^{j}=\zeta_{i}^{j}, \text { for } i=2,3, \ldots, r_{j} \text { and } j=1,2, \ldots, p, \\
& {\left[\dot{\zeta}_{r_{1}}^{1}, \dot{\zeta}_{r_{2}}^{2}, \ldots, \dot{\zeta}_{r_{p}}^{p}\right]^{\mathrm{T}}=E \beta+M \zeta+\Psi u+N w,} \\
& e=\left[\zeta_{1}^{1}, \zeta_{1}^{2}, \ldots, \zeta_{1}^{p}\right]^{\mathrm{T}} .
\end{aligned}
$$

In the new coordinate $(\beta, \zeta)$, it can be explicitly observed which control signal should be generated by the designed controller to achieve the output regulation objective. The 
following theorem presents the solution of the differential regulator equation based on the new coordinates.

Theorem 4.3.3 [55] Under Assumptions 3-6, the linear differential matrix equation

$$
\dot{\Pi}^{\beta}(t)+\Pi^{\beta}(t) S(t)=\Gamma \Pi^{\beta}(t)+\Theta
$$

always has a solution $\Pi^{\beta}(t)=\left[\left(\Pi^{\beta a}(t)\right)^{\mathrm{T}} \quad\left(\Pi^{\beta s}(t)\right)^{\mathrm{T}}\right]^{\mathrm{T}}$, which can be obtained as follows,

$$
\begin{aligned}
& \Pi^{\beta a}(t)=-\int_{t}^{\infty} \Phi_{\Gamma^{a}}(t, \sigma) \Theta^{a} \Phi_{s}(\sigma, t) d \sigma \\
& \Pi^{\beta s}(t)=\Phi_{\Gamma^{s}}\left(t, t_{0}\right) \Pi_{0}^{\beta s} \Phi_{s}\left(t_{0}, t\right)+\int_{t_{0}}^{t} \Phi_{\Gamma^{s}}(t, \sigma) \Theta^{s} \Phi_{s}(\sigma, t) d \sigma
\end{aligned}
$$

where $\Theta^{a}$ and $\Theta^{s}$ are the upper $k$ rows and the lower $n-k-r$ rows of $\Theta$, respectively. $\Pi_{0}^{\beta s}$ is any constant $(n-r-k) \times q$ matrix. The DRE (4.23) has a solution given as

$$
\begin{aligned}
& \Pi=\left(T^{x}\right)^{-1}\left(\left[\begin{array}{c}
\Pi^{\beta} \\
0_{r \times q}
\end{array}\right]-T^{w}\right), \\
& R=-(\Psi)^{+}\left(E \Pi^{\beta}+N\right),
\end{aligned}
$$

where $(\Psi)^{+}$is the right-inverse of $\Psi$.

Note that the solution to the DRE for the stable zero dynamics $\Pi^{\beta s}$ can be found by calculating the corresponding definite integral with finite limits in Theorem 4.3.3. On the other hand, the solution to the anti-stable part of the zero dynamics $\Pi^{\beta a}$ is more difficult to obtain, and the infinite upper limit of the closed-form solution can lead to numerical errors during the implementation. In the remainder of this section, an alternative approach is presented to Theorem 4.3.3, and a numerically robust suboptimal solution is developed to the output regulation problem. 
The bounded solution for the stable part of the zero dynamics can be easily obtained by iterating the following DRE

$$
\dot{\Pi}^{\beta s}(t)=\Gamma^{s} \Pi^{\beta s}(t)-\Pi^{\beta s}(t) S(t)+\Theta^{s}
$$

with some arbitrary initial condition $\Pi^{\beta s}\left(t_{0}\right)$. However, it is not as straightforward to solve the unstable part of the zero dynamics for the DRE.

In order to guarantee a bounded solution, the unstable zero dynamics of (4.30) is first stabilized. Assuming that a gain $K_{r}$ exists such that all the eigenvalues of $\left(\Gamma+\Lambda K_{r}\right)$ are exponentially stable, rewrite (4.30a) as

$$
\dot{\beta}=\left(\Gamma+\Lambda K_{r}\right) \beta+\Lambda\left(\zeta-K_{r} \beta\right)+\Theta w
$$

Let $\tilde{\zeta}=\zeta-K_{r} \beta$ and Equation (4.30) then becomes

$$
\begin{aligned}
\dot{\beta} & =\left(\Gamma+\Lambda K_{r}\right) \beta+\Lambda \tilde{\zeta}+\Theta w \\
\dot{\tilde{\zeta}}_{i-1}^{j} & =\tilde{\zeta}_{i}^{j}, \text { for } i=2,3, \cdots, r_{j} \text { and } j=1,2, \ldots, p \\
{\left[\dot{\tilde{\zeta}}_{r_{1}}^{1}, \dot{\tilde{\zeta}}_{r_{2}}^{2}, \ldots, \dot{\tilde{\zeta}}_{r_{p}}^{p}\right]^{\mathrm{T}} } & =E \beta+M \tilde{\zeta}+\Psi u+N w-K_{r}\left(\Gamma \beta+\Lambda\left(\tilde{\zeta}+K_{r} \beta\right)+\Theta w\right), \\
& =\left(E-K_{r} \Gamma+M K_{r}-K_{r} \Lambda K_{r}\right) \beta+\left(M-K_{r} \Lambda\right) \tilde{\zeta}+\Psi u+\left(N-K_{r} \Theta\right) w \\
e & =\left[\tilde{\zeta}_{1}^{1}, \tilde{\zeta}_{1}^{2}, \ldots, \tilde{\zeta}_{1}^{p}\right]^{\mathrm{T}}+K_{r} \beta .
\end{aligned}
$$

Based on the updated normal form (4.34), let the control input $u(t)$ drive the states $\tilde{\zeta}$ to zero. The regulated error then becomes $e=K_{r} \beta$. In addition, for the zero dynamics in (4.34a), the solution to the corresponding DRE

$$
\dot{\Pi}^{\beta}(t)=\left(\Gamma+\Lambda K_{r}\right) \Pi^{\beta}(t)-\Pi^{\beta}(t) S(t)+\Theta
$$


is always bounded. As $\tilde{\zeta} \rightarrow 0$, the system equation (4.34) reduces to

$$
\begin{aligned}
\dot{\beta} & =\left(\Gamma+\Lambda K_{r}\right) \beta+\Theta w, \\
e & =K_{r} \beta
\end{aligned}
$$

To regulate the residual error in (4.35b), the $H_{2}$ norm of the transfer function from $w$ to $e$, given by $G_{e}(s)=K_{r}\left(s I-\Gamma-\Lambda K_{r}\right)^{-1} \Theta$, is minimized.

The unified gradient method from [58] is utilized to minimize the $H_{2}$ norm of (4.35). Assuming that $\Gamma+\Lambda K_{r}$ is stable, the $H_{2}$ norm of $G_{e}(s)$ is given by

$$
\left\|G_{e}(s)\right\|_{2}^{2}=\operatorname{tr}\left(\Theta^{\mathrm{T}} P \Theta\right)=: J_{1}
$$

where $P=P^{\mathrm{T}} \geq 0$ satisfies the Lyapunov equation

$$
\left(\Gamma+\Lambda K_{r}\right)^{\mathrm{T}} P+P\left(\Gamma+\Lambda K_{r}\right)=-K_{r}^{\mathrm{T}} K_{r}
$$

In the case where the poles of $\Gamma+\Lambda K_{r}$ are not fixed a priori, the gain $K_{r}$ may become unbounded to make the $H_{2}$ norm in (4.36) small. Instead, the following $H_{2}$ optimization problem under pole assignment constraints is considered

$$
\begin{aligned}
& \inf _{K_{r}} J_{1}\left(K_{r}\right), \\
& \text { s.t. } V^{-1}\left(\Gamma+\Lambda K_{r}\right) V=\Lambda_{r},
\end{aligned}
$$

where $\Lambda_{r}$ is a real block-diagonal matrix with its eigenvalues corresponding to the desired pole locations, $V$ is a nonsingular matrix satisfying $\Gamma V-V \Lambda_{r}=-\Lambda U$ and $K_{r}=U V^{-1}$ for a free parameter $U$. 
For the constrained optimization (4.38), it was shown in [58] that

$$
\frac{\partial J_{1}}{\partial K_{r}}=2 \Lambda^{\mathrm{T}} P X
$$

where $X$ is the unique solution to the Lyapunov equation

$$
\left(\Gamma+\Lambda K_{r}\right) X+X\left(\Gamma+\Lambda K_{r}\right)^{\mathrm{T}}=-\Theta \Theta^{\mathrm{T}}
$$

Furthermore, the gradient of the objective function about the free parameter matrix $U$ was given by

$$
\frac{\partial J_{1}}{\partial U}=\frac{\partial J_{1}}{\partial K_{r}}\left(V^{-1}\right)^{\mathrm{T}}+\Lambda^{\mathrm{T}} Y^{\mathrm{T}}
$$

where $\partial J_{1} / \partial K_{r}$ is given in (4.39), and $Y$ is the unique solution to the Sylvester equation

$$
Y \Gamma-\Lambda_{r} Y=V^{-1}\left(\frac{\partial J_{1}}{\partial K_{r}}\right)^{\mathrm{T}} K_{r} .
$$

A local minimum to (4.38) can then be found numerically by evaluating the gradient (4.41) about the free variable $U$.

The complete process of the unified gradient method is illustrated in Fig. 4.1.

\subsection{Summary}

This chapter presents the theoretical fundamentals of the dissertation. The TPF control law is first introduced to handle LTI systems subject to input delay and the stability conditions for the input-delay system under the TPF control law are established. Afterwards, the output regulation problem is presented for input-delay systems with a time-invariant exosystem and an output feedback solution is constructed based on the TPF control law and static regulator gains. Finally, based on the differential regulator equation, the output regulation problem extends to input-delay systems with time-varying exosystem. A sub-optimal solution 




Figure 4.1: The unified gradient method process.

is developed for non-minimum phase systems so that the regulator gains are always bounded and the residual error in the regulated output is locally minimized. 


\section{Chapter 5}

\section{Unbalance Compensation under Constant Rotational Speeds}

\subsection{TPF control for the AMB test rig}

In the first section of this chapter, the TPF control method presented in Section 4.1 is employed to construct the rotor levitation controller for the flexible rotor AMB test rig described in Section 3.1 in the presence of input delay. Furthermore, the control law maximizing the upper bound of the delay that the stable closed-loop system can tolerate is derived.

\subsubsection{Maximization of the delay bound}

A linear matrix inequality (LMI) method is used to derive the TPF control law that satisfies the stability conditions (4.9) for the maximum delay bound $\bar{D}[59]$. Given that the conditions (4.9) are nonlinear with respect to the decision variables $W, \alpha, \omega$, and $\bar{D}$, the path-following method [60] is used to find a local solution to the original nonlinear optimization problem by solving a linearized approximation. The linearized decision variables are considered, which are defined as $W=W_{0}+\delta W, \alpha=\alpha_{0}+\delta \alpha, \omega=\omega_{0}+\delta \omega$, and $\bar{D}=\bar{D}_{0}+\delta \bar{D}$. The known variables $W_{0}, \alpha_{0}, \omega_{0}$, and $\bar{D}_{0}$ are the initial solutions satisfying the original matrix inequalities 
(4.9). The perturbation terms $\delta W, \delta \alpha, \delta \omega$, and $\delta \bar{D}$ are the decision variables of the linearized LMI problem, and they correspond to an incremental improvement of the solution at each iteration of the path-following method. After eliminating higher order perturbation terms, the linearized LMI problem becomes

$$
\begin{aligned}
& \alpha_{0} \delta W+W_{0} \delta \alpha+\alpha_{0} W_{0}-B B^{T}>0, \\
& A \delta W+\delta W A^{T}-\delta \omega W_{0}-\omega_{0} \delta W \\
& +A W_{0}+W_{0} A^{T}-\omega_{0} W_{0}<0, \\
& \left(A W+W A^{T}-B B^{T}+\frac{h_{1}}{\omega_{0}} \alpha_{0}^{3} \bar{D}_{0} W_{0}\right) \\
& +\left(A \delta W+\delta W A^{T}+\frac{h_{1}}{\omega_{0}} \alpha_{0}^{3} \bar{D}_{0} \delta W\right) \\
& +\left(\frac{h_{2}}{\omega_{0}} \bar{D}_{0} \alpha_{0}^{3} W_{0}-\frac{h_{1}}{\omega_{0}} \bar{D}_{0} \alpha_{0}^{3} W_{0}\right) \delta \omega \\
& +W_{0}\left(\frac{h_{1}}{\omega_{0}} \alpha_{0}^{3}+h_{2} \alpha_{0}^{3} \bar{D}_{0}\right) \delta \bar{D} \\
& +\left(3 \frac{h_{1}}{\omega_{0}} \bar{D}_{0} \alpha_{0}^{2} W_{0}\right) \delta \alpha<0, \\
& {\left[\begin{array}{cc}
h_{\text {step }} W_{0} & \delta W \\
\delta W^{T} & h_{\text {step }} W_{0}
\end{array}\right]>0,} \\
& {\left[\begin{array}{cc}
h_{\text {step }} \bar{D}_{0} & \delta \bar{D} \\
\delta \bar{D} & h_{\text {step }} \bar{D}_{0}
\end{array}\right]>0 \text {, }} \\
& {\left[\begin{array}{cc}
h_{\text {step }} \alpha_{0} & \delta \alpha \\
\delta \alpha & h_{\text {step }} \alpha_{0}
\end{array}\right]>0 \text {, }} \\
& {\left[\begin{array}{cc}
h_{\text {step }} \omega_{0} & \delta \omega \\
\delta \omega & h_{\text {step }} \omega_{0}
\end{array}\right]>0,}
\end{aligned}
$$

where $h_{1}=e^{2 \omega_{0} \bar{D}_{0}}-e^{\omega_{0} \bar{D}_{0}}, h_{2}=2 e^{2 \omega_{0} \bar{D}_{0}}-e^{\omega_{0} \bar{D}_{0}}$, and $h_{\text {step }}>0$. The inequalities (5.1a) (5.1c) result from the linearization of the conditions in (4.9). Conditions (5.1d) - (5.1g) aim 
to limit the maximum step sizes of the perturbation terms.

Each iteration of the path-following method starts with an initial feasible solution to (4.9), and solves for the linearized LMI problem (5.1). At the end of each iteration, a test is carried out before moving on to the next iteration to check if the found solution satisfies the original inequality (4.9). If satisfied, the initial feasible solution is updated. If not satisfied, the initial feasible solution is left unchanged and $h_{\text {step }}$ is reduced.

To avoid the solution converges to an undesired local minimum, an objective function $J_{i}$ is defined in which the objective is switched at each step of the path-following iteration as

$$
J_{i}= \begin{cases}-\delta \bar{D} & \text { for } i \bmod 3=0 \\ \delta \omega & \text { for } i \bmod 3=1 \\ \delta \alpha & \text { for } i \bmod 3=2\end{cases}
$$

where $i$ is the iteration number. The first objective increases the delay bound by solving for the maximum $\delta \bar{D}$; the second and third objectives indirectly not only steer the solution towards a larger delay bound by increasing $\omega$ and $\alpha$, but also keep the solution of the path-following algorithm out of an undesired shallow local minimum.

The complete optimization procedure for the LMI method is illustrated in Fig. 5.1.

\subsubsection{Controller derivation for the AMB test rig}

To simplify the design of the TPF control law, the lateral control axes of the AMB systems are assumed to be decoupled, which is equivalent to the condition that there is no gyroscopic effect for a stationary rotor. The dynamic equation of the AMB system is given in the following state space form

$$
\begin{aligned}
& \dot{\zeta}(t)=A \zeta(t)+B u(\phi(t)), \\
& z(t)=C \zeta(t),
\end{aligned}
$$




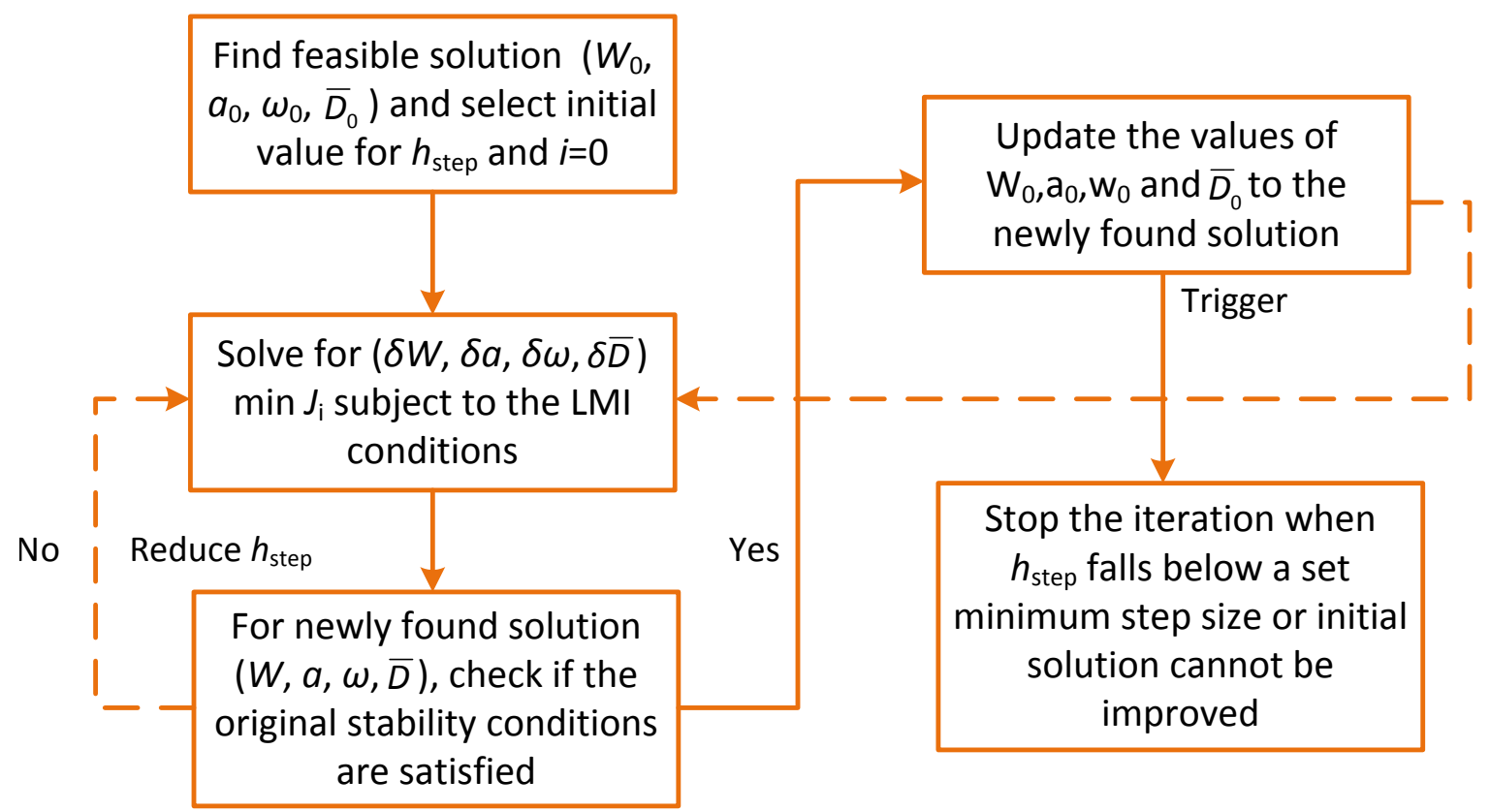

Figure 5.1: The LMI-based optimization procedure for determining the maximal allowable delay bound.

where $u(t)$ is the control signal to the current amplifiers powering the AMB actuators and the output $z(t)$ contains rotor displacements as observed at the locations of the sensors. A notch filter at $1005 \mathrm{~Hz}$ is added to the control loop at each output channel of the plant in order to attenuate the excitation of the rotor third bending mode. This mode is outside the range of the AMB dynamic force envelope, and it is common practice in AMB systems to filter out the high frequency modes of the plant. The input delay is considered to be constant

$$
\phi(t)=t-D
$$

for some $D>0$. The objective is to find a TPF controller corresponding to the largest $\bar{D}$ such that the AMB system is stable for any $D \leq \bar{D}$.

The initial feasible solution set for the LMI equations is $\alpha_{0}=1000, \omega_{0}=1000$, and $\bar{D}_{0}=6 \times 10^{-6} \mathrm{~s}$. The path-following algorithm with initial $h_{\text {step }}=0.2$ is used to derive the control law that locally minimizes the objective function (5.2). The solution to the 


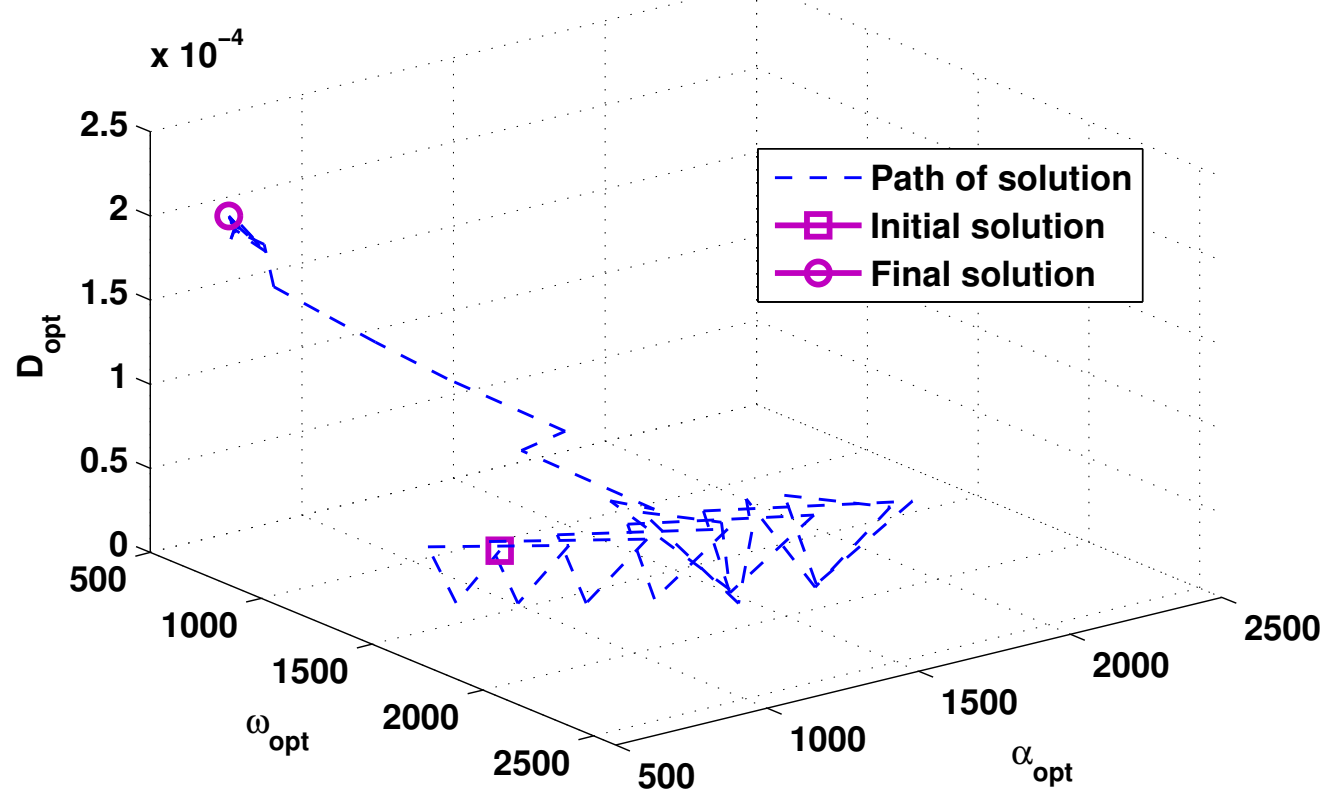

Figure 5.2: Path of the optimal solution.

LMI approximation in (5.1) is obtained using the MATLAB software package SDPT3 [61], which has been observed to have good numerical stability in this particular application. The final solution set from the path-following algorithm is $\alpha_{0}=652.58, \omega_{0}=638.74$, and $\bar{D}_{0}=2.02 \times 10^{-4} \mathrm{~s}$.

Figure 5.2 shows the trajectory of the optimal solution at each iteration of the pathfollowing method. It is observed that the solution of the path-following method initially wanders in searching for a path towards a desired optimal solution. Without the perturbation terms added in the objective function as in (5.2), the solution would quickly converge to an undesired "shallow" local minimum, before finding a path towards a more desirable solution. The added perturbation terms enable the solution to escape the pull of undesired local minima and drive it towards a more desired solution.

To implement the state feedback TPF control law on the AMB test rig, a state observer is constructed based on a Kalman filter. By combining the TPF control law with the state 


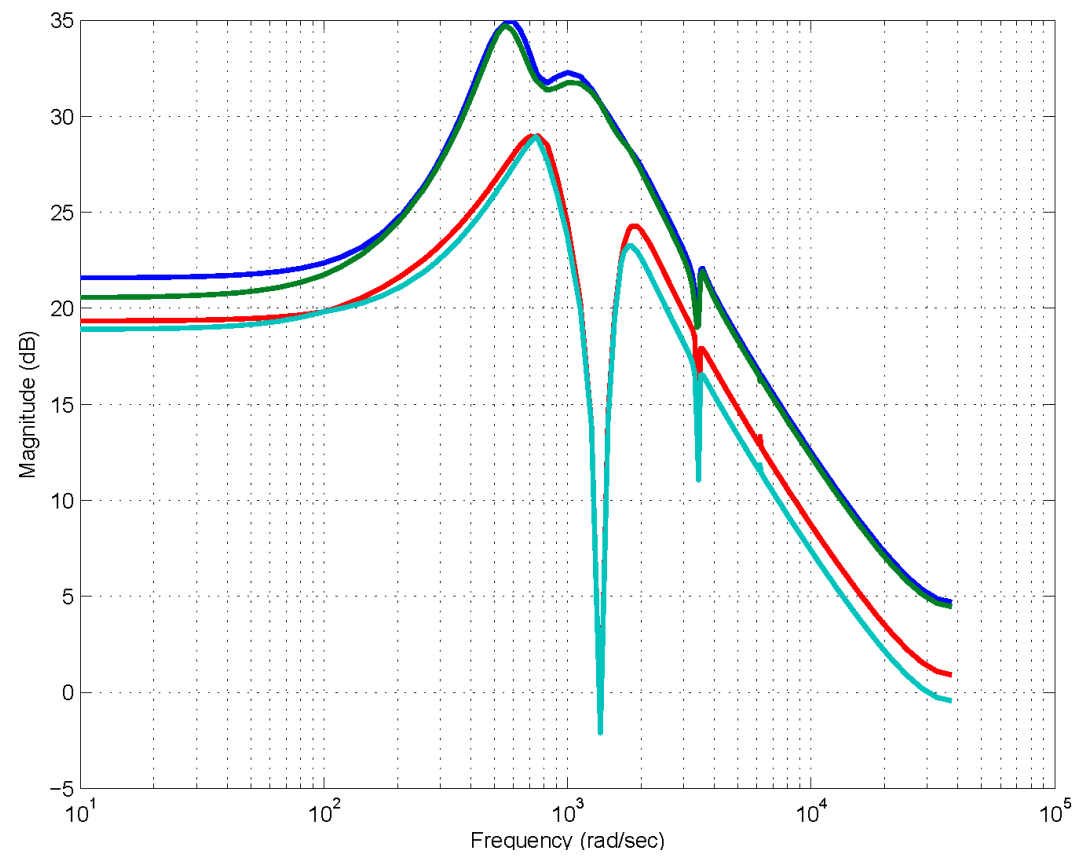

Figure 5.3: Singular value plot of the derived output feedback TPF controller.

observer of the AMB system, the dynamic output feedback controller is obtained as

$$
\begin{aligned}
\dot{\hat{\zeta}}(t) & =(A+L C) \hat{\zeta}(t)+B u(\phi(t))-L z(t), \\
u(t) & =K e^{A D} \hat{\zeta}(t) .
\end{aligned}
$$

The stability of the linear time delay system under the above output feedback TPF control was proven in [25]. The observer gain $L$ of the Kalman filter is obtained following the standard procedure such that it minimizes the steady state error covariance of the observed states. The Control System Toolbox in MATLAB is utilized to calculate such gain, where the covariance values for the inputs and outputs of the plant are $5 \times 10^{-2}$ and $1 \times 10^{-5}$, respectively. Meanwhile, the design of the observer is not affected by the delay and the observer error dynamics can be decoupled from the design of the state feedback TPF control. The singular value plot of the final controller with $D=\bar{D}$ is shown in Fig. 5.3. 


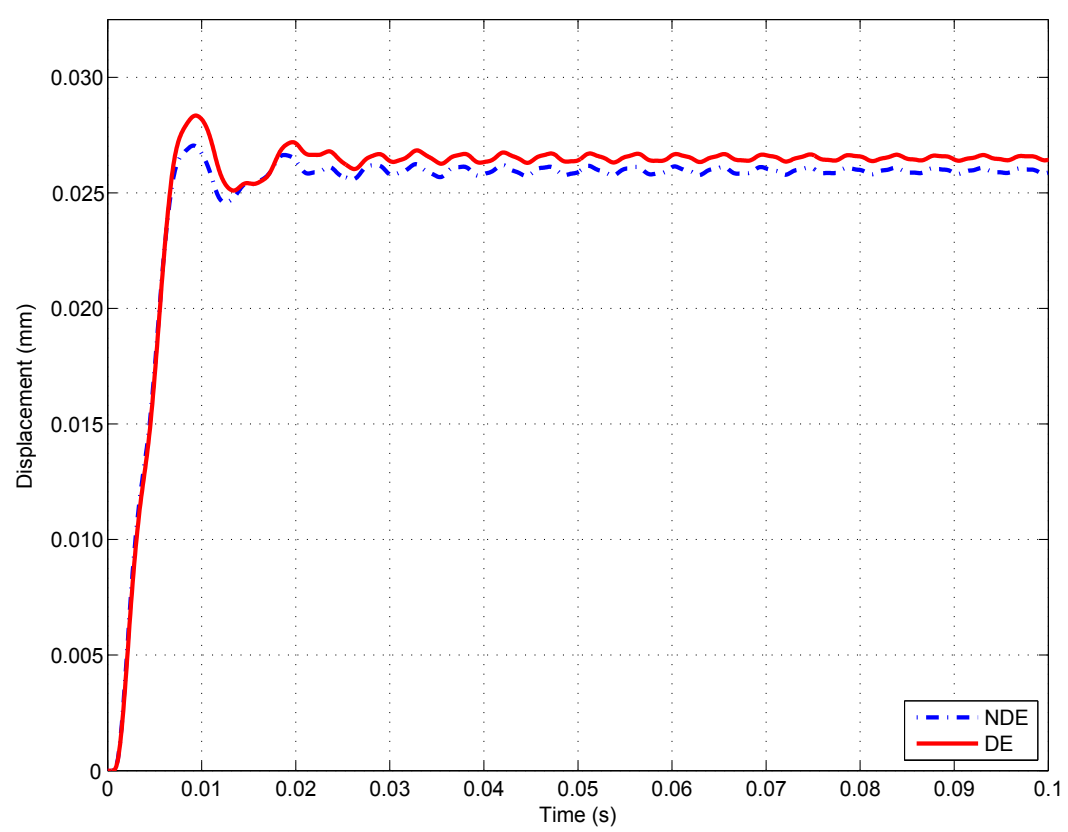

Figure 5.4: Simulated step response of the closed-loop AMB system with a constant input delay.

\subsubsection{Simulation results}

The control law derived in Section 4.1 is first verified through simulation. The plant model is obtained from the dynamic equations of the AMB system as described in Section 3.2. A constant input delay (5.4) is introduced to the simulation, with $D=2.02 \times 10^{-4} \mathrm{~s}$.

Fig. 5.4 shows the step response of the closed-loop system, in which an step reference of $0.0254 \mathrm{~mm}$ is fed to the closed-loop AMB system at both the driven and non-driven ends. The step response indicates that the AMB system is stable with good transient performance. Small oscillations can be observed at the beginning for the rotor displacement due to the excitation of the first bending mode, but the displacement eventually settles to the steady state.

Afterwards, a rotor unbalance is added at the disk location in order to induce a synchronous disturbance on the rotor and the unbalance response test is performed for the speed to gradually increase from 0 to $7000 \mathrm{rpm}$. The zero-to-peak magnitude of the rotor vibration during the rotor spin-up is plotted in Fig. 5.5. The figure shows that the AMB system 


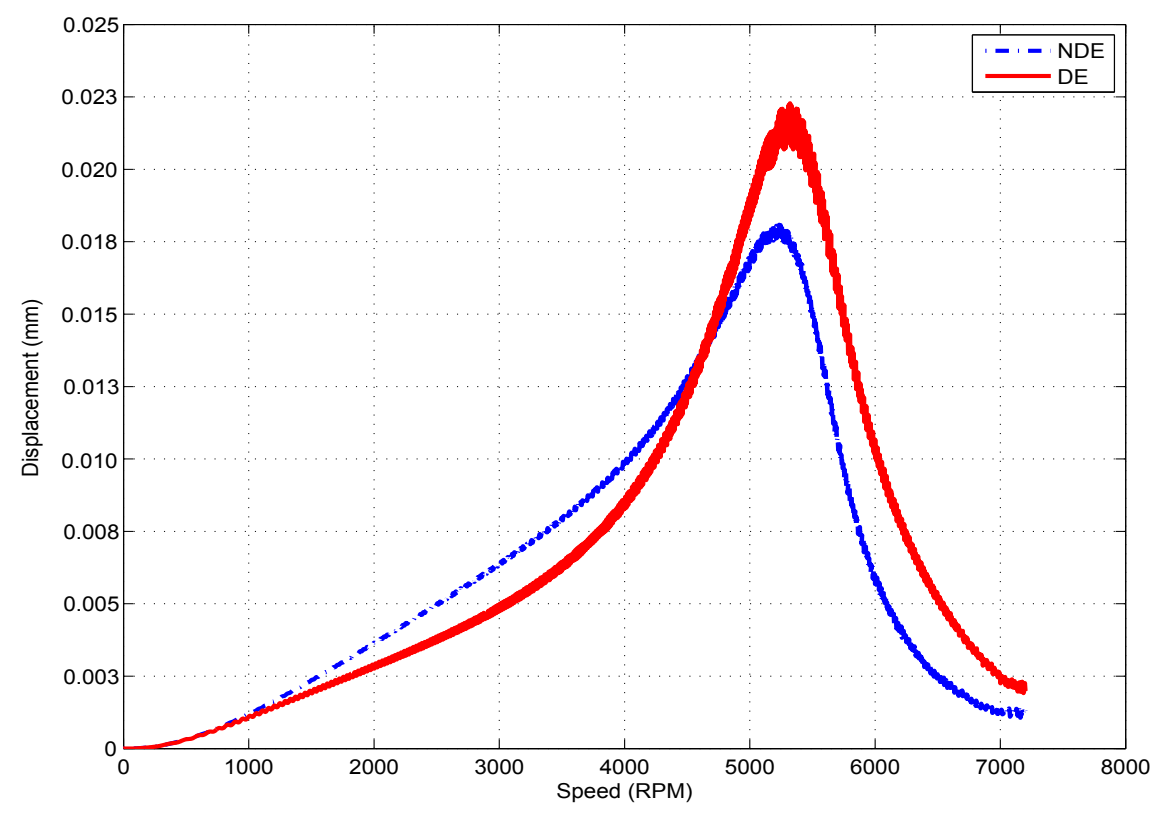

Figure 5.5: Simulated zero-to-peak rotor vibration level for rotational speeds up to $7000 \mathrm{rpm}$.

remains stable within the tested speed range, and the amplitude of the vibration is contained within the levels specified by the various industry standards for high speed rotating machines. The peak vibrations located near 5,000 rpm come from the resonance of the rotor second rigid body mode.

\subsubsection{Experimental results}

The TPF control law was implemented and tested on the flexible rotor AMB test rig as described in Section 3.1. In order to recreate the delay experienced in remotely controlled machinery, a time delay was added in the digital controller between the calculation of the controller output and the transmission of the command signal to the current amplifiers powering the AMB actuators.

Prior to the implementation of the optimal TPF controller for experimental testing, special considerations were required regarding some of the physical limitations of the test setup. A lightly weighted integrator was added to the controller to reduce the steady-state error in the rotor position due to the static gravitational force. Furthermore, in order to prevent 


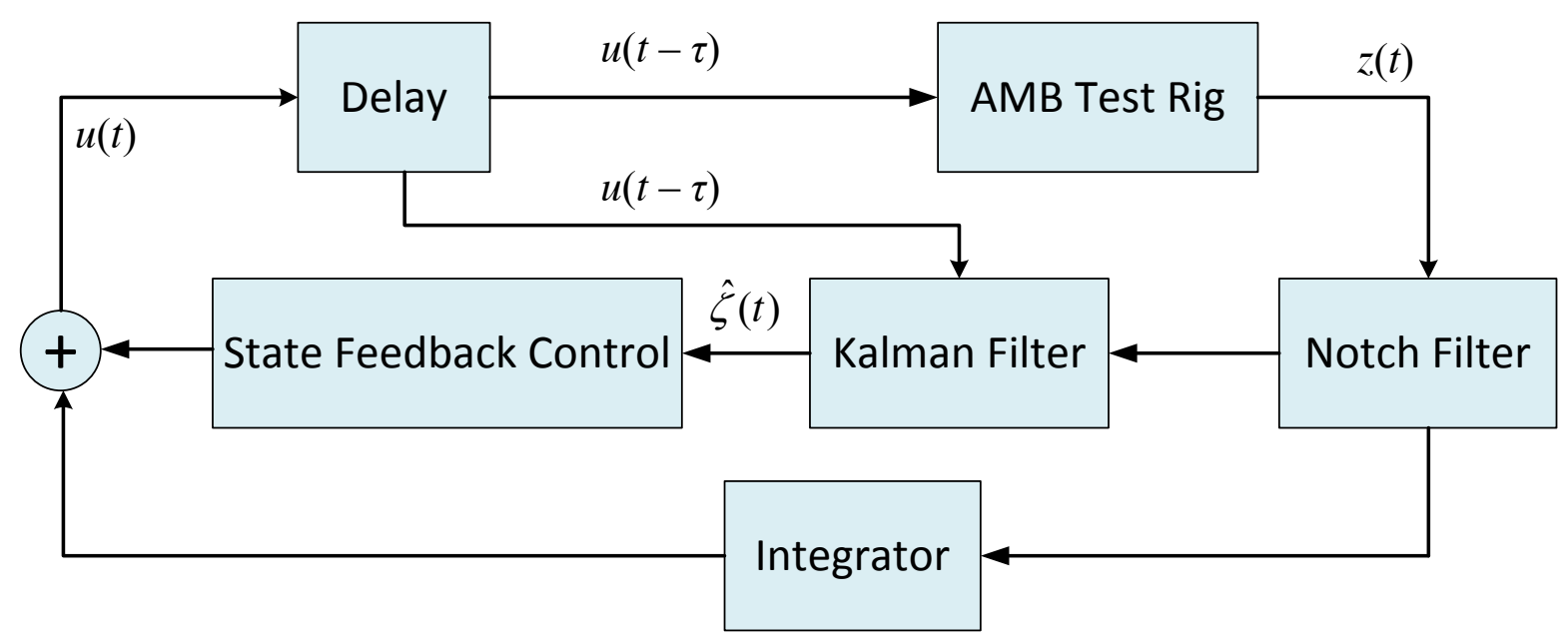

Figure 5.6: Controller feedback loop implementation in the AMB test rig.

actuator saturation and maintain the AMB operation within the dynamic force envelope of the bearings, as well as to prevent the excitation of the higher order modes of the flexible rotor, the $H_{\infty}$ norm and bandwidth of the control law were constrained. This was achieved in this case by carefully selecting the gains of the output feedback observer. Finally, in order to reduce the computational load, the order of the final controller was reduced to 14 states per lateral axis, or 28 states in total, using the Hankel singular value based model reduction function reduce in MATLAB.

With the special considerations given above, a TPF controller was derived and implemented on the test rig. A block diagram describing the implementation of the control loop for the AMB test rig is shown in Fig. 5.6. Note that because the delay in the input was implemented in the digital controller, the length of the time delay is always a multiple of the control sampling time $T_{s}=8.33 \times 10^{-5} \mathrm{~s}$.

First based on the delay bound $\bar{D}$ corresponding to the optimized controller, the input delay is set to be $D=3 T_{s}$ and the initial rotor levitation was tested. For comparison, the levitation test was first performed with the $\mu$-synthesis controller presented in [5], which was carefully designed for the AMB test rig to provide the best robustness and performance. It 


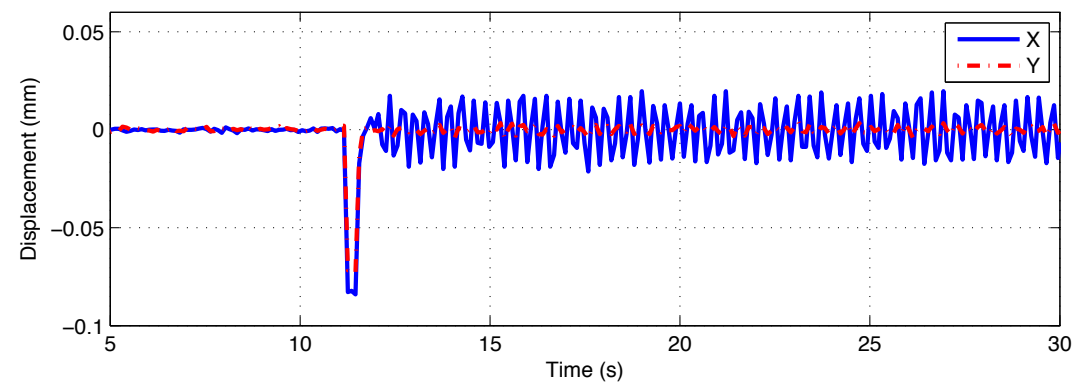

(a) Driven end

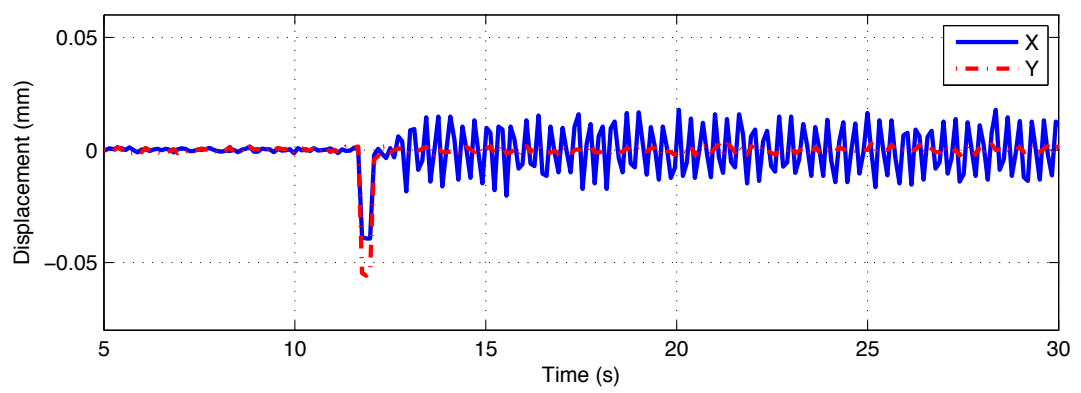

(b) Non driven end

Figure 5.7: Rotor displacement measurement during levitation with $\mu$-synthesis controller and input delayed by $D=3 T_{\mathrm{s}}$.

can be observed from Fig. 5.7 that some of the modes are close to instability as indicated by the high amplitude rotor vibration. The same test was performed on the test rig using the TPF controller. As illustrated in Fig. 5.8, the rotor levitation is stable as expected and the rotor gradually returns to the zero positions after the initial transient response.

The maximum delay estimated by the LMI based method was conservative since longer delay can be tolerated during simulation. To further investigate the observation experimentally, the delay was gradually increased and the rotor levitation test was performed correspondingly. When $D=8 T_{s}$, it is observed that the rotor levitation is stable from Fig. 5.9, and the transient response is more noticeable since the delayed input signal takes longer time to reach the AMBs. When $D=10 T s$, it is observed that the test rig starts showing instability as in Fig. 5.10.

The unbalance response of the AMB system with input delay $D=6 T_{s}$, or equivalently $5 \times 10^{-4} \mathrm{~s}$ was carried out up to a speed near 5,000 rpm. The zero-to-peak vibration amplitude 


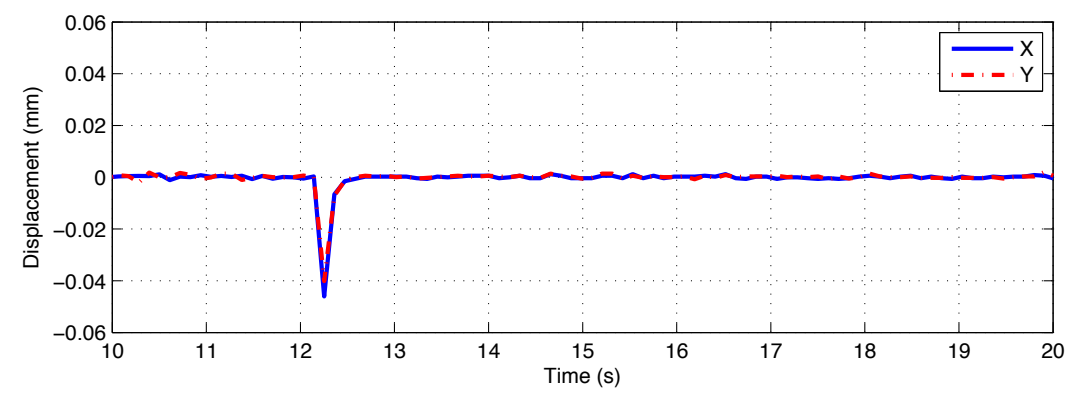

(a) Driven end

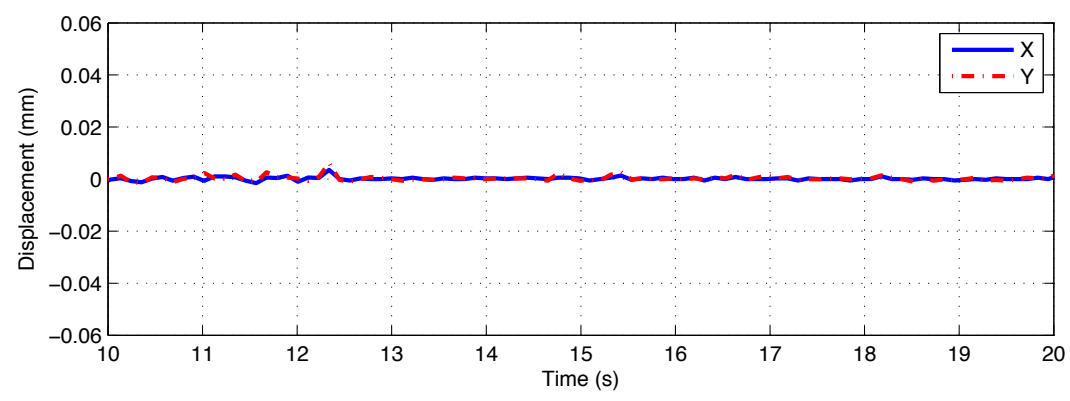

(b) Non driven end

Figure 5.8: Rotor displacement measurement during levitation with TPF controller and input delayed by $D=3 T_{\mathrm{s}}$.

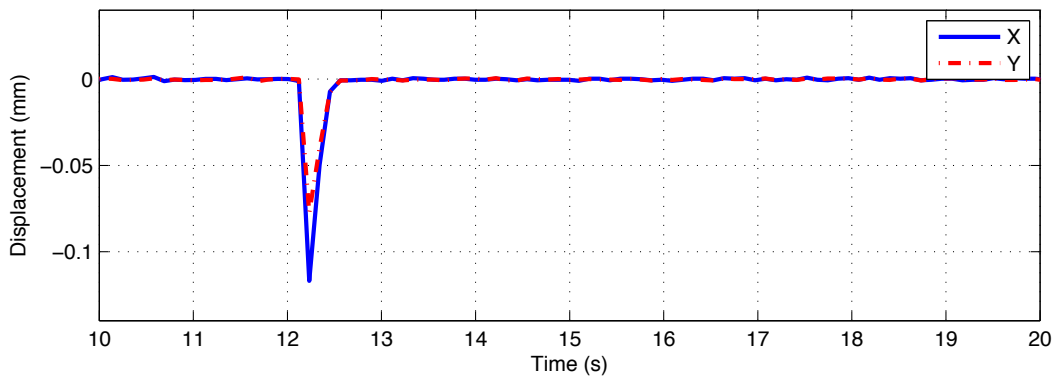

(a) Driven end

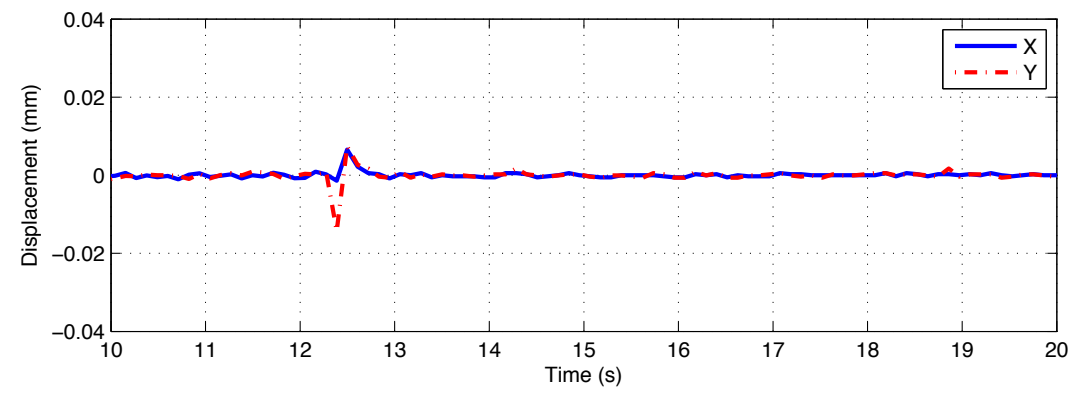

(b) Non driven end

Figure 5.9: Rotor displacement measurement during levitation with TPF controller and input delayed by $D=8 T_{\mathrm{s}}$. 


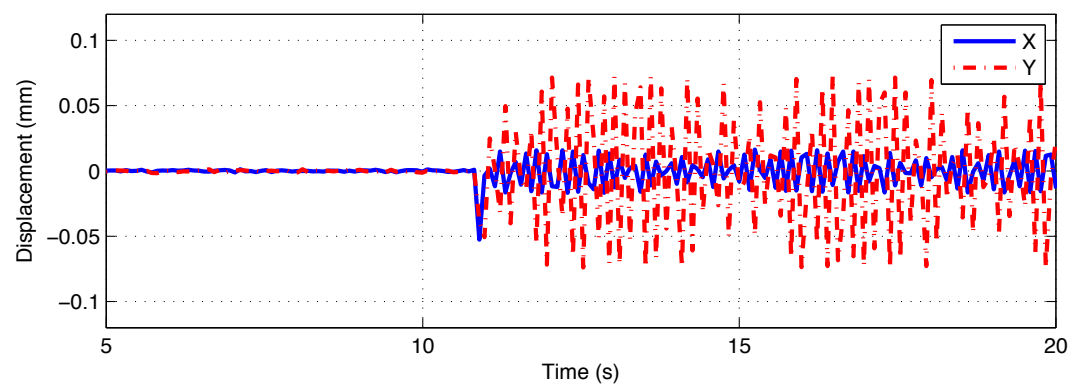

(a) Driven end

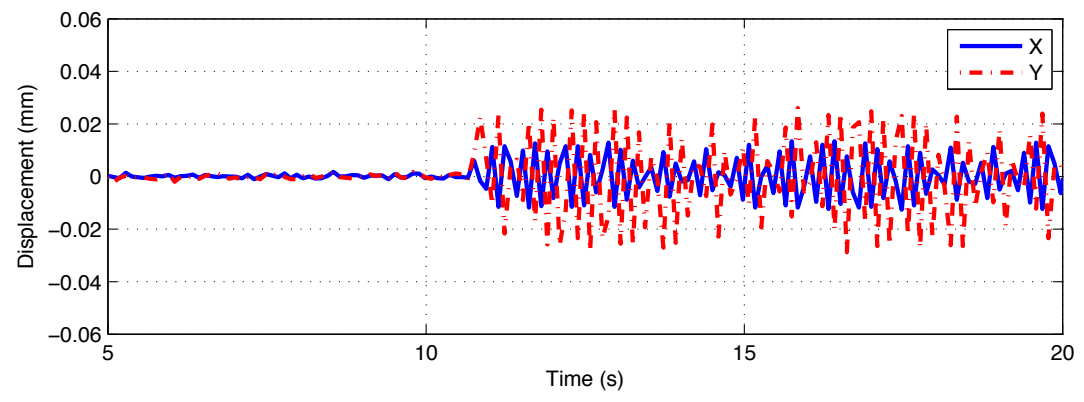

(b) Non driven end

Figure 5.10: Rotor displacement measurement during levitation with TPF controller and input delayed by $D=10 T_{\mathrm{s}}$.

of the rotor is shown in terms of the rotational speed. The results from Fig. 5.11 show that the rotor vibration is well contained and the vibration level is within the operation zones suggested for long-term operating by various industrial specifications. For example, the American Petroleum Institute (API) [62] and the International Organization of Standardization (ISO) [63] require the zero-to-peak vibration level below $0.075 \mathrm{~mm}$, or 2.95 mils for similar turbomachineries.

The rotating speed is limited to 5,000 rpm during the experimental test because it is observed that the rotor first bending mode is easily excited as the rotor speed approaches the corresponding resonance frequency, which is also observed during the numerical simulation in Section 5.1.3. As the rotor speed approaches the critical speed of the AMB system, large oscillations appear in the rotor position and the control signal, which may cause instability and serious damage to the test rig. The difficulty in compensating for the excitation of the rotor modes is mainly due to the speed dependent dynamics of the rotor, which introduces a 


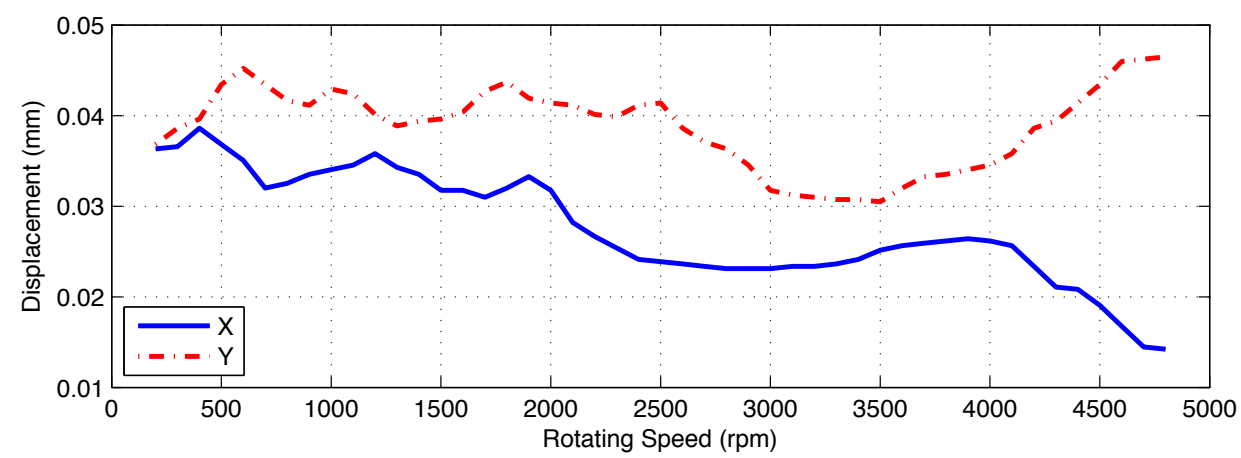

(a) Driven end

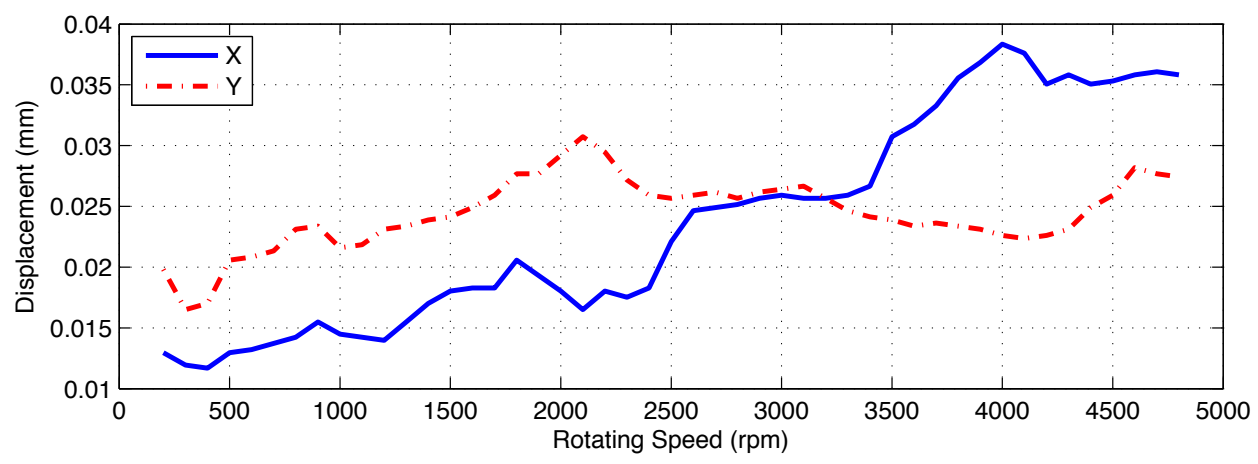

(b) Non driven end

Figure 5.11: Zero-to-peak rotor vibration amplitude measured at different rotating speeds with TPF controller and input delayed by $D=6 T_{\mathrm{s}}$.

significant uncertainty to the LTI AMB system model.

\subsection{Unbalance compensation in AMB systems}

In this section, the unbalance compensation problem is investigated for AMB systems with input delays using the output regulation approach proposed in Section 4.2. The unbalance compensation method is based on the solution to an equivalent output regulation problem. Since precise location and eccentricity of a rotor unbalance are difficult to measure in high speed rotating machines, the unbalance forces and the rotor displacements are defined as the errors to be regulated, which enables the AMB system to achieve autobalancing and unbalance suppression. The developed model based unbalance compensation controller is first verified in simulations and then demonstrated experimentally that it is able to notably 
reduce the synchronous rotor vibrations and the magnitude of the AMB control voltage.

\subsubsection{Problem formulations}

The net force generated by the pair of AMB actuators can be approximated by

$$
f_{\mathrm{amb}}=K_{\mathrm{x}} x+K_{\mathrm{i}} i_{\mathrm{c}}
$$

where $K_{\mathrm{x}}$ is the open loop stiffness, $K_{\mathrm{i}}$ is the open loop gain of the AMB, $i_{c}$ is the perturbation current and $x$ is the rotor displacement at the AMB location.

The lateral dynamic equation of the AMB system with input delay, under the assumption that the rotor and AMB actuators are symmetric regarding both $x$ and $y$ lateral axes, can be expressed in the following state space form

$$
\begin{aligned}
{\left[\begin{array}{c}
\dot{\zeta}_{x}(t) \\
\dot{\zeta}_{y}(t)
\end{array}\right]=} & {\left[\begin{array}{cc}
A_{\zeta} & \omega G \\
-\omega G & A_{\zeta}
\end{array}\right]\left[\begin{array}{c}
\zeta_{x}(t) \\
\zeta_{y}(t)
\end{array}\right]+\left[\begin{array}{cc}
B_{\mathrm{c}} & 0 \\
0 & B_{\mathrm{c}}
\end{array}\right]\left[\begin{array}{l}
i_{\mathrm{c}, x}(t-\tau) \\
i_{\mathrm{c}, y}(t-\tau)
\end{array}\right] } \\
& +\sum_{j=1}^{s}\left[\begin{array}{cc}
B_{\mathrm{d}, j} & 0 \\
0 & B_{\mathrm{d}, j}
\end{array}\right]\left[\begin{array}{l}
w_{x, j}(t) \\
w_{y, j}(t)
\end{array}\right]
\end{aligned}
$$

where the subscripts $x$ and $y$ refer to the lateral $x$ and $y$ directions of the AMB system, respectively, $i_{c}$ is the control current to the AMBs, and $\omega$ represents the rotating speed. The state vector $\zeta$ describes the lateral dynamics of the AMB system, which includes the AMB actuators, AMB electronics and rotor. The lateral dynamics of the rotor are commonly modeled through finite-element methods, and a modal coordinate transformation is employed to simplify the resulting differential equation. The matrices $A_{\zeta} \in \mathbf{R}^{20 \times 20}$ and $B_{\mathrm{c}} \in \mathbf{R}^{20 \times 2}$ are the state space matrices of a single axis AMB system, and the lateral axes are coupled by the gyroscopic effect $\omega G . w_{x, j}$ and $w_{y, j}$ represent the disturbance force generated by the $j^{\text {th }}$ 
unbalance mass on the rotor in the following form

$$
\begin{aligned}
& w_{x, j}=\epsilon_{j} \omega^{2} \cos \left(\omega t+\theta_{j}\right), \\
& w_{y, j}=\epsilon_{j} \omega^{2} \sin \left(\omega t+\theta_{j}\right),
\end{aligned}
$$

where $\epsilon_{j}$ is the unbalance eccentricity and $\theta_{j}$ is the phase angle. Then they can be modeled in the following dynamic form as the exosystem

$$
\left[\begin{array}{c}
\dot{w}_{x, j} \\
\dot{w}_{y, j}
\end{array}\right]=\left[\begin{array}{cc}
0 & -\omega \\
\omega & 0
\end{array}\right]\left[\begin{array}{l}
w_{x, j} \\
w_{y, j}
\end{array}\right] .
$$

The AMB system and the exosystem can be rewritten in the following standard output regulation problem form

$$
\begin{aligned}
\dot{\zeta}(t) & =\mathbf{A} \zeta(t)+\mathbf{B} i_{\mathrm{c}}(t-\tau)+\sum_{j=1}^{s} \mathbf{P}_{j} w_{j}(t), \\
\dot{w}_{j}(t) & =\mathbf{S} w_{j}, \text { for } j=1,2, \cdots, s
\end{aligned}
$$

where

$$
\begin{aligned}
& \zeta(t)=\left[\begin{array}{l}
\zeta_{x}(t) \\
\zeta_{y}(t)
\end{array}\right], i_{\mathrm{c}}(t-\tau)=\left[\begin{array}{l}
i_{\mathrm{c}, x}(t-\tau) \\
i_{\mathrm{c}, y}(t-\tau)
\end{array}\right], w_{j}(t)=\left[\begin{array}{l}
w_{x, j}(t) \\
w_{y, j}(t)
\end{array}\right], \\
& \mathbf{A}=\left[\begin{array}{cc}
A_{\zeta} & \omega G \\
-\omega G & A_{\zeta}
\end{array}\right], \mathbf{B}=\left[\begin{array}{cc}
B_{\mathrm{c}} & 0 \\
0 & B_{\mathrm{c}}
\end{array}\right], \mathbf{P}_{j}=\left[\begin{array}{cc}
B_{\mathrm{d}, j} & 0 \\
0 & B_{\mathrm{d}, j}
\end{array}\right], \mathbf{S}=\left[\begin{array}{cc}
0 & -\omega \\
\omega & 0
\end{array}\right] .
\end{aligned}
$$

In developing the unbalance compensation controller, it is assumed that the rotor is a rigid body so the design is focused on the vibration patterns composed of the rigid body modes of the rotor. The parallel mode describes the translation of the rotor about the center line while the conical mode describes the rotor tilt about its center of mass. In a sub-critical 
AMB system operation, the rotor vibration combines the effect from the parallel and conical modes.

In order to simultaneously regulate both parallel and conical modes of the rotor, two locations along the rotor length are selected where the disturbances $\omega_{j}$ 's are assumed to be applied. These locations are selected so they do not coincide with the nodal points of the rotor modes under consideration. In the case where higher order modes of the rotor are considered, additional disturbance input locations may be introduced. For simplicity, the locations of the disturbance forces are defined to match the locations of the AMB actuators. Based on this information, the matrices $\mathbf{P}_{1}$ and $\mathbf{P}_{2}$ can be found during the modeling of the rotor dynamics.

In this section, two scenarios of output regulations are considered. In the first case, the error signal is the rotor displacement measured at the sensor locations and it can be defined from the system states as

$$
e_{d}(t)=\mathbf{C}_{\mathrm{sens}} \zeta(t)
$$

where $\mathbf{C}_{\text {sens }}$ is the output matrix. By regulating the rotor displacement, the observed rotor vibration is reduced and the rotor is forced to rotate about the geometric center.

The second case is to regulate the AMB forces and thus it reduces the disturbance forces generated by the unbalance mass. The error signal is defined to be the applied AMB forces as

$$
e_{f}(t)=K_{\mathrm{x}} \mathbf{C}_{\mathrm{amb}} \zeta(t)+K_{\mathrm{i}} i_{c}(t-\tau)
$$

Once again, the matrix $\mathbf{C}_{\mathrm{amb}}$ is obtained such that $\mathbf{C}_{\mathrm{amb}} \boldsymbol{\zeta}$ corresponds to the rotor displacements at the bearing locations. In this case, the AMB forces are regulated, which in turn reduces the disturbance forces generated by the unbalance mass on the rotor. Based on (5.8), the unbalance force is zero only if the unbalance eccentricity equals zero,

$$
\epsilon_{j}=0, \text { for } j=1,2, \cdots, s,
$$




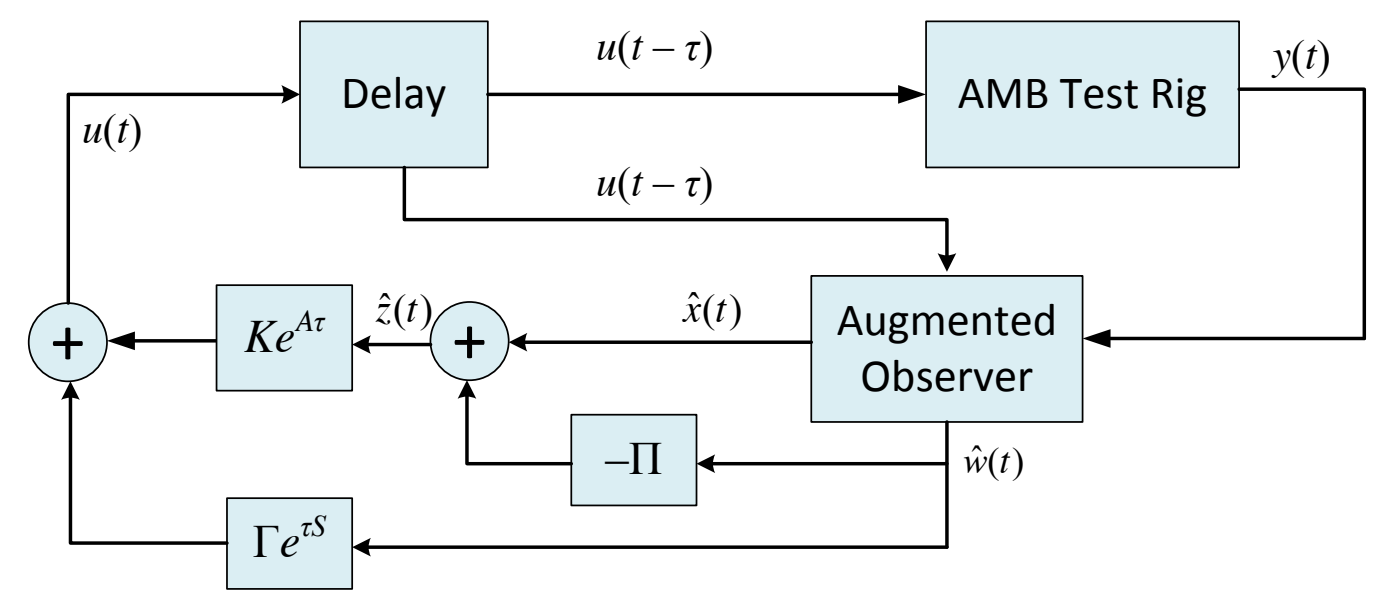

Figure 5.12: Block diagram of the controller implementation under constant rotational speed.

and the rotor rotates about its center of mass. Therefore, this second case corresponds to a compensation of the rotor unbalance.

Based on the validated AMB system model, the rotor levitation controller and the unbalance compensation methods are designed. The leviation controller is based on the TPF control method for the undisturbed case $(\omega \equiv 0)$ as detailed in Section 5.1. The results of Lemma 4.2.2 yield an augmented observer to estimate the states of the plant and the exosystem. The augmented observer is utilized to construct the output feedback controller and the block diagram of the final implemented controller is illustrated in Fig. 5.12.

\subsubsection{Simulation and experimental results}

The output regulation control designed to regulate the error signals (5.12) and (5.11) for the AMB test rig were first verified through simulation. The simulation of the AMB system included two unbalance masses located at the locations of the bearings. The rotational speed was fixed at 6,000 rpm and the input delay was set to be $\tau=0.5 \mathrm{~ms}$. First, the rotor displacement regulation control was tested as shown in Fig. 5.13. The AMB system is initially controlled by the levitation controller designed for the undisturbed system, and the output 

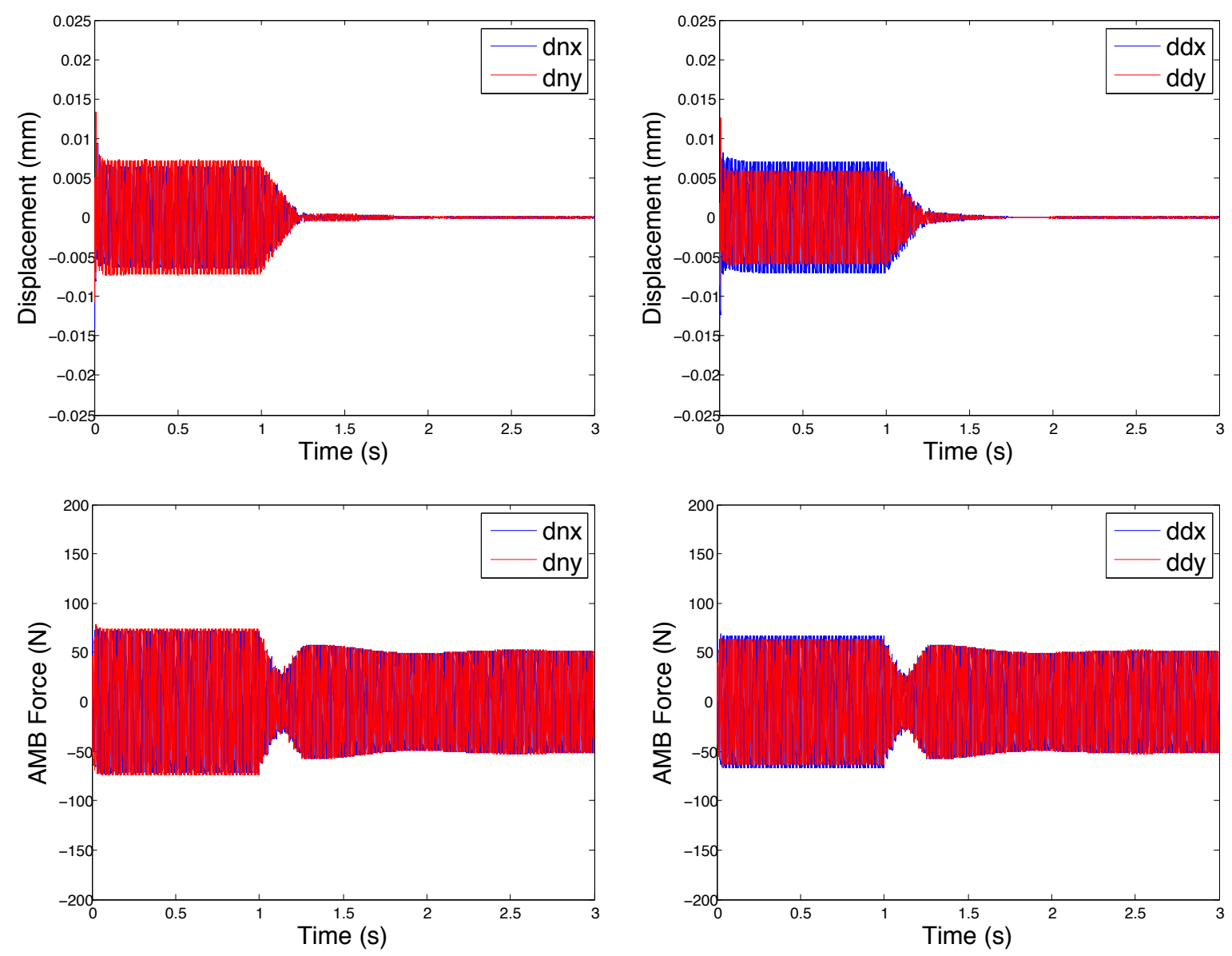

Figure 5.13: Simulated rotor displacements and AMB forces under the rotor displacement regulation with the output regulator active for $t>1 \mathrm{~s}$.

regulator component is activated near time $t=1 \mathrm{~s}$. As expected, the amplitude of the rotor vibration approaches zero after the output regulator is activated.

Then the AMB force regulator was tested and similar results are obtained as shown in Fig. 5.14, where the amplitude of the AMB forces converges to zero after the unbalance compensator is activated.

The output regulation control laws were also implemented and tested on the ROMAC flexible rotor AMB test rig. As in the simulation, the rotational speed is set to $6,000 \mathrm{rpm}$ and input delay $\tau=0.5 \mathrm{~ms}$. The eccentricity and axial distribution of the rotor unbalance are unknown, and they can be determined by the residual error during the initial rotor balancing process. 

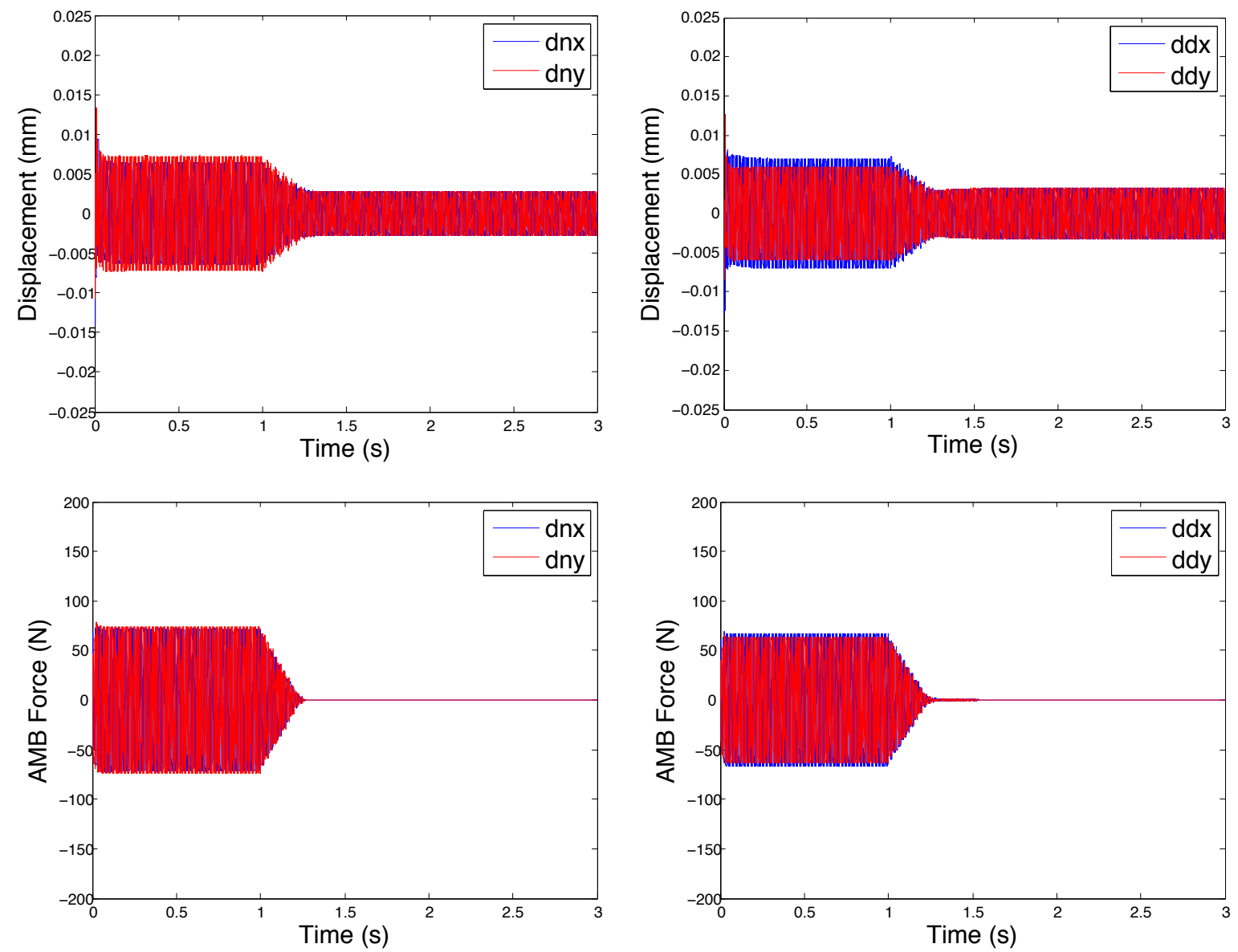

Figure 5.14: Simulated rotor displacements and AMB forces under the AMB force regulation with the output regulator active for $t>1 \mathrm{~s}$.

Fig. 5.15 presents the results corresponding to the base controller designed for the AMB system without the regulator to evaluate the performance of the output regulation controllers. The first row of Fig. 5.15 show the control voltage from the controller to the current amplifiers powering the AMB actuators; the second row of Fig. 5.15 shows the rotor vibrations measured by the sensors at the bearing locations; the rotor obits at the bearing locations are illustrated in the third row of Fig. 5.15.

Fig. 5.16 presents the results corresponding to the controller designed for the AMB system with the rotor displacement regulation. The first row of Fig. 5.16 shows the control voltage from the controller to the current amplifiers powering the AMB actuators; The second row of Fig. 5.16 shows the rotor vibrations measured by the sensors at the bearing locations; 

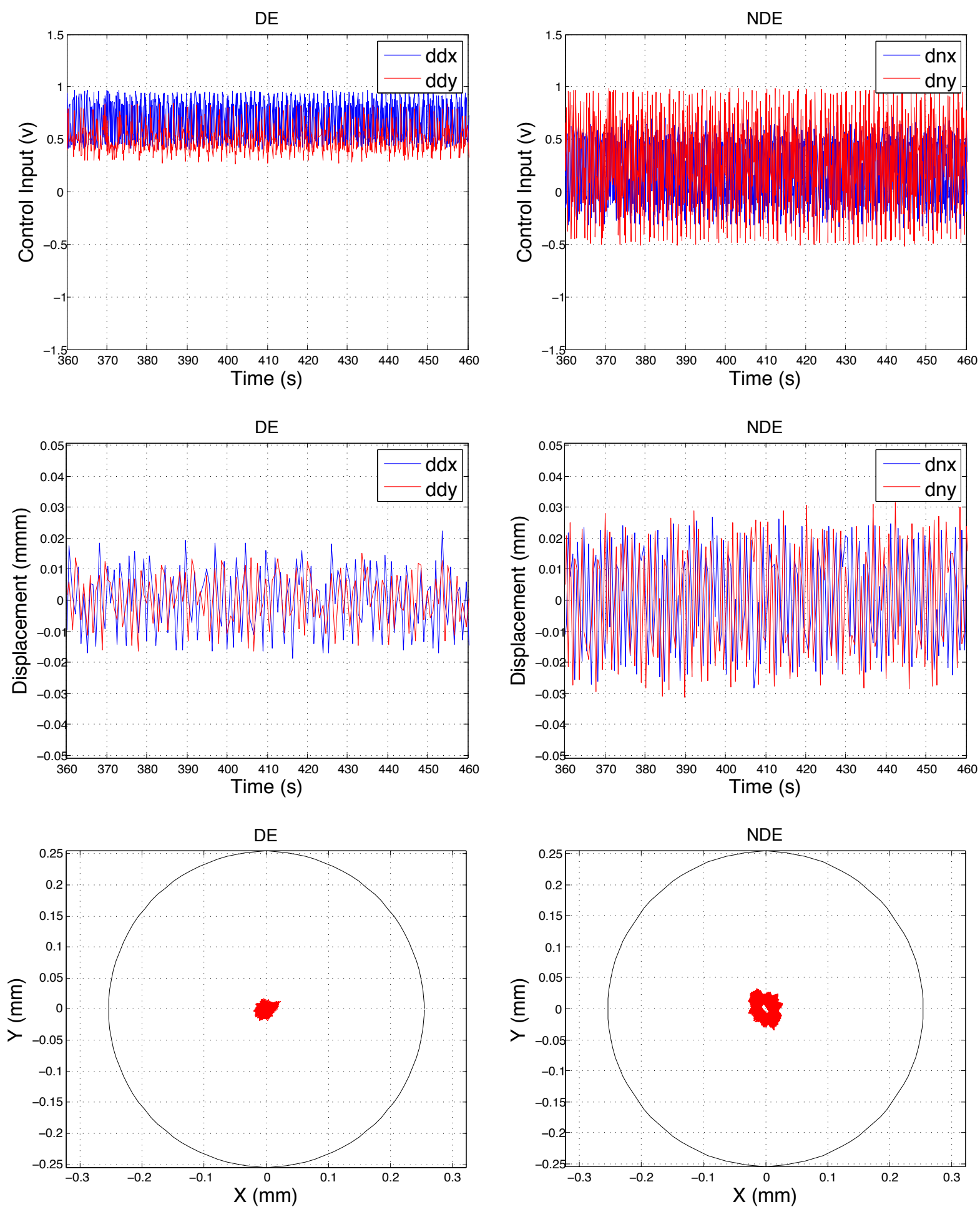

Figure 5.15: Experimental measurements under the controller designed for the non-regulated system. 

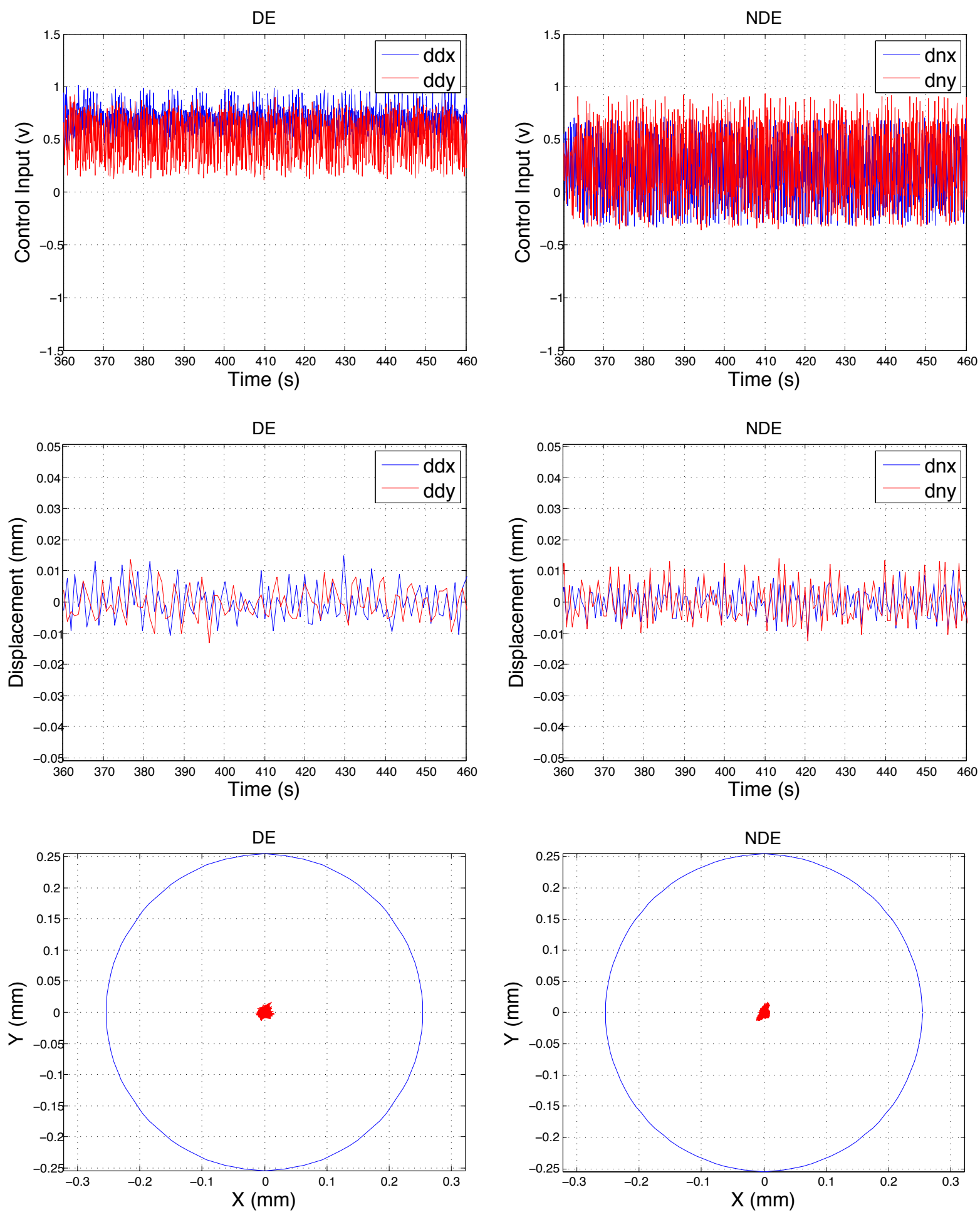

Figure 5.16: Experimental measurements under the controller with the rotor displacement regulation. 


\begin{tabular}{|l|l|l|l|l|l|l|l|l|}
\hline & $\begin{array}{l}\text { DEX } \\
\text { Vib/mm } \\
(\mathrm{pk}-\mathrm{pk})\end{array}$ & $\begin{array}{l}\text { DEY } \\
\text { Vib/mm } \\
(\mathrm{pk}-\mathrm{pk})\end{array}$ & $\begin{array}{l}\text { DEX } \\
\text { Ctl/v } \\
(\mathrm{pk}-\mathrm{pk})\end{array}$ & $\begin{array}{l}\text { DEY } \\
\text { Ctl/v } \\
(\mathrm{pk}-\mathrm{pk})\end{array}$ & $\begin{array}{l}\text { NDEX } \\
\text { Vib/mm } \\
(\mathrm{pk}-\mathrm{pk})\end{array}$ & $\begin{array}{l}\text { NDEY } \\
\text { Vib/mm } \\
(\mathrm{pk}-\mathrm{pk})\end{array}$ & $\begin{array}{l}\text { NDEX } \\
\text { Ctl/v } \\
\text { (pk-pk) }\end{array}$ & $\begin{array}{l}\text { NDEY } \\
\text { Ctl/v } \\
(\mathrm{pk}-\mathrm{pk})\end{array}$ \\
\hline $\begin{array}{l}\text { Without } \\
\text { regulation }\end{array}$ & 0.0413 & 0.0316 & 0.5779 & 0.5826 & 0.0552 & 0.0629 & 1.0874 & 1.5233 \\
\hline $\begin{array}{l}\text { Vibration } \\
\text { regulator }\end{array}$ & 0.0257 & 0.0266 & 0.6644 & 0.8082 & 0.0214 & 0.0264 & 1.0462 & 1.3011 \\
\hline $\begin{array}{l}\text { Force } \\
\text { regulator }\end{array}$ & 0.0206 & 0.0296 & 0.3411 & 0.4492 & 0.0280 & 0.0270 & 0.4016 & 0.5126 \\
\hline
\end{tabular}

Table 5.1: Performance summary of the experimental results.

the rotor obits at the bearing locations are illustrated in the third row of Fig. 5.16. It is clear that the rotor vibration level has been significantly reduced when compared to the no regulation case and the control voltages also show smaller amplitude oscillations compared to the no regulation case but the improvement is not as significant.

Fig. 5.17 presents the results corresponding to the controller designed for the AMB system with the AMB force regulation. The first row of Fig. 5.17 shows the control voltage from the controller to the current amplifiers powering the AMB actuators; the second row of Fig. 5.17 shows the rotor vibrations measured by the sensors at the bearing locations; the rotor obits at the bearing locations are illustrated in the third row of Fig. 5.17. The measured rotor displacement at the sensor locations demonstrate that the rotor vibration level is well suppressed when compared to the no regulation case. The control voltages are significantly reduced, which indicates the energy required to compensate for the unbalance force has decreased since the rotating axis closely coincides with the the rotor principle axis of inertia.

The performance summaries of the experimental results at different control channels are shown in Table 5.1. It is observed that with the displacement regulator implemented, the vibration is reduced up to $61 \%$ while the control voltage remains in the same range compared to no regulation case; meanwhile, with the force regulator implemented, the vibration is reduced up to $57 \%$ and the control voltage is reduced up to $66 \%$ compared to no regulation case. The experimental results demonstrate that the proposed unbalance compensation methods are effective in suppressing the rotor vibration and reducing the peak control 

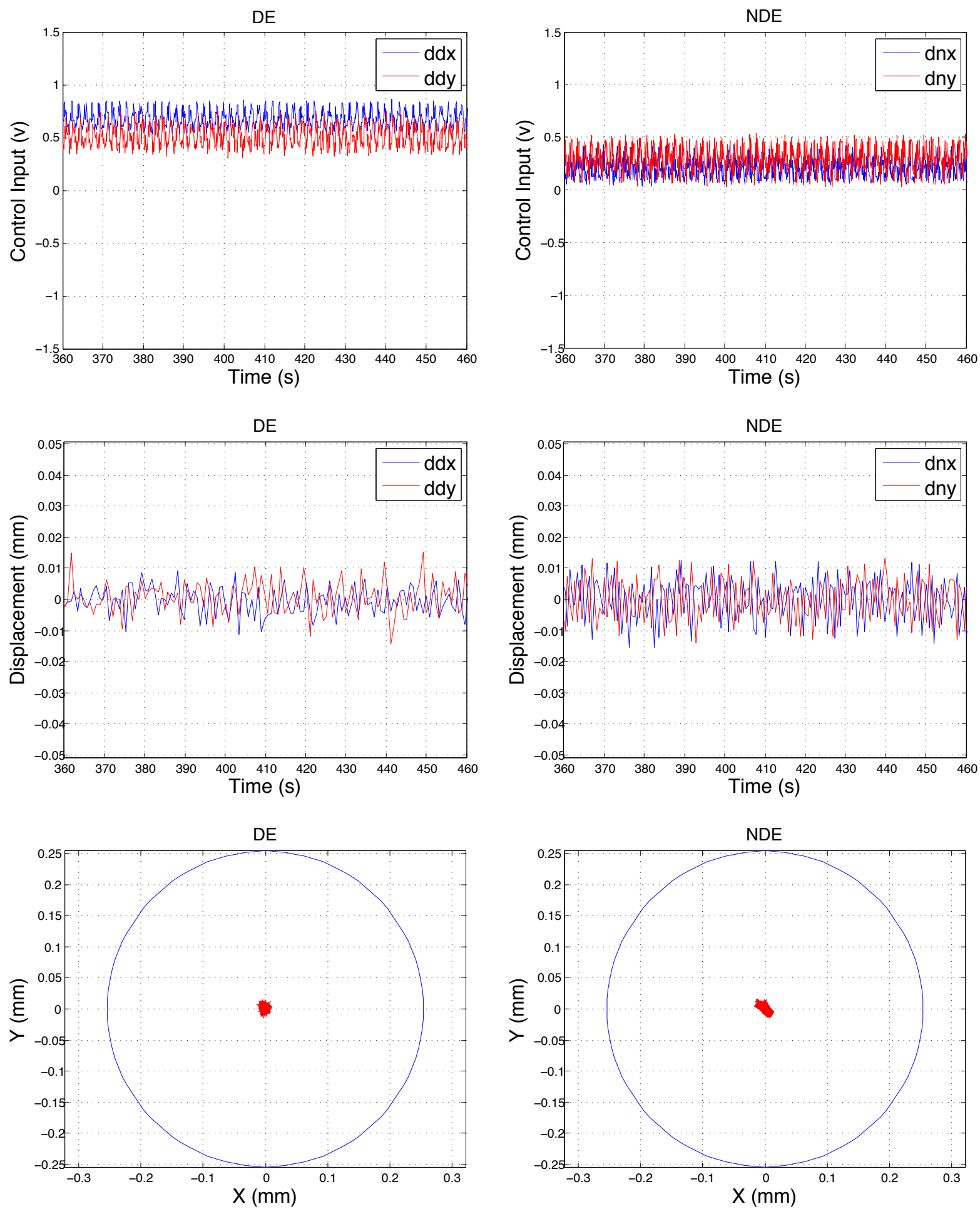

Figure 5.17: Experimental measurements under the controller with the AMB force regulation. 
voltage.

\subsection{Summary}

This chapter presents the unbalance compensation for an AMB test rig subject to input delay under constant rotational speed. First of all, the TPF control law is applied to construct the rotor levitation controller and an LMI method is adopted to maximize the upper bound of the delay that the stable closed-loop system can tolerate. The stability and performance of the closed-loop AMB system under the designed controller is first verified through simulation, and then demonstrated on the AMB test rig. The TPF controller notably outperforms a previously designed $\mu$-synthesis robust controller when longer input delay is added and the rotor vibration level is sufficiently contained during the unbalance response test. Besides, it is observed that the delay bound estimated by the proposed optimization method is conservative. Afterwards, the output regulation and TPF based control method is applied to the AMB test rig to achieve unbalance compensation under constant rotational speed. Two output regulation scenarios are investigated, which define the unbalance forces and rotor displacements as the regulation errors. Both simulation and experimental results reveal that the synchronous rotor vibrations and the magnitude of the AMB control voltage can be significantly reduced using the developed controllers. 


\section{Chapter 6}

\section{Unbalance Compensation under Time-varying Rotational Speeds}

\subsection{Autobalancing in an AMB system}

In the first section of this chapter, the output regulation based unbalance compensation approach is applied to the AMB test rig with time-varying rotational speeds. After formulating the output regulation problem with a time-varying exosystem as detailed in Section 4.3, it becomes evident that the compensator gains can be obtained based on the solution of a differential regulator equation (DRE). Since AMB systems are of non-minimum phase, to ensure the boundedness of the compensator gains, the original normal form is reformulated and the unified gradient method is adopted to guarantee that suboptimal solutions are achieved. Then the compensator gains are generated iteratively to enable the closed-loop system to approach the output regulation objective with a locally minimized residual error in the regulated output. To apply the output regulation mechanism to AMB systems for autobalancing, the unbalance force is modeled by the exosystem and the AMB force defines the error to be regulated [64].

Because the test rotational speed is far away from the rotor flexible mode frequencies, the 

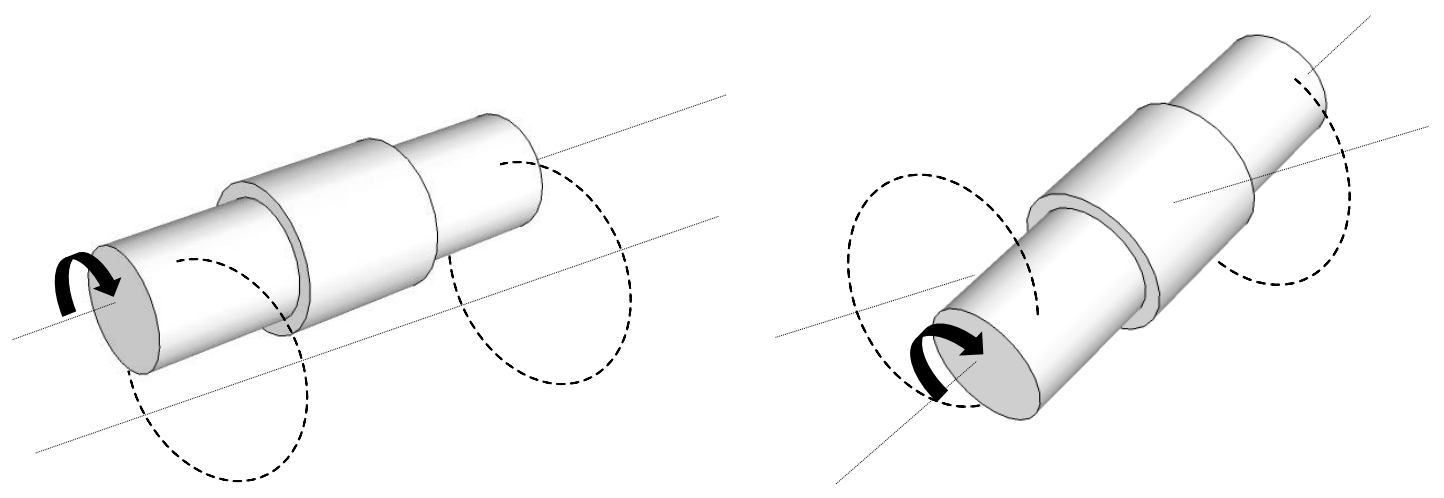

Figure 6.1: Rotor rigid body modes.

rotor is assumed to be a rigid body in designing the autobalancing controller. Therefore, only the rigid body modes of the rotor are considered. When the AMB system operates below the frequency of the first flexible mode, the rotor vibration profile is mainly dominated by the two rigid body modes. The parallel mode is characterized by the rotor translation around the center line while the conical mode describes the rotor tilt around its center of mass as shown in Fig. 6.1. In order to regulate both rotor rigid modes independently, two locations defining the disturbance force entrance are identified in the AMB system model. Since only the rigid modes are considered, the actual locations of the disturbance forces are not important as long as they are not collocated. For simplicity, the matrices are specified for the disturbance at the locations that match the locations of the AMB actuators.

The lateral dynamic equation of a rotor AMB system is described as follows,

$$
\left[\begin{array}{c}
\dot{\zeta}_{x}(t) \\
\dot{\zeta}_{y}(t)
\end{array}\right]=\left[\begin{array}{cc}
A_{\zeta} & \omega G_{r} \\
-\omega G_{r} & A_{\zeta}
\end{array}\right]\left[\begin{array}{l}
\zeta_{x}(t) \\
\zeta_{y}(t)
\end{array}\right]+\left[\begin{array}{cc}
B_{c} & 0 \\
0 & B_{c}
\end{array}\right]\left[\begin{array}{c}
i_{c, x}(t-\tau) \\
i_{c, y}(t-\tau)
\end{array}\right]+\sum_{j=1}^{s}\left[\begin{array}{cc}
B_{d, j} & 0 \\
0 & B_{d, j}
\end{array}\right]\left[\begin{array}{c}
w_{x, j}(t) \\
w_{y, j}(t)
\end{array}\right],
$$

where the subscripts $x$ and $y$ refer to the lateral $x$ and $y$ directions of the AMB system, respectively, $i_{c}$ is the control current to the AMBs, $\zeta$ describes the states of the AMB system lateral dynamics, $\omega$ is the rotating speed, $G_{r}$ is the gyroscopic matrix, and $w_{x, j}$ and $w_{y, j}$ represent the time-varying disturbance force generated by the $j_{\text {th }}$ unbalance mass on the 
rotor in the following form

$$
\begin{gathered}
w_{x, j}=\epsilon_{j} \omega^{2} \cos \left(\omega t+\theta_{j}\right), \\
w_{y, j}=\epsilon_{j} \omega^{2} \sin \left(\omega t+\theta_{j}\right),
\end{gathered}
$$

where $\epsilon_{j}$ is the unbalance eccentricity, $\omega$ is a random time-varying rotational speed and $\theta_{j}$ is the phase angle. Then the disturbance forces can be modeled by the following time-varying exosystem,

$$
\left[\begin{array}{c}
\dot{w}_{x, j} \\
\dot{w}_{y, j}
\end{array}\right]=\left[\begin{array}{cc}
0 & -\omega(t) \\
\omega(t) & 0
\end{array}\right]\left[\begin{array}{l}
w_{x, j} \\
w_{y, j}
\end{array}\right] .
$$

To achieve autobalancing under time-varying unbalance forces, the rotational axis should consistently adjust itself and dynamically approach the principle axis of inertia. Then the rotor performs force-free rotation and the support AMB forces are significantly reduced. To apply the output regulation mechanism, the regulated error signal is defined as the applied AMB forces

$$
f_{\mathrm{amb}}(t)=K_{x} C_{\mathrm{amb}} \zeta(t)+K_{i} i_{c}(t-\tau)
$$

where $i_{c}$ is the control current, and $K_{x}$ and $K_{i}$ are the open loop stiffness and the current gain of the AMB, respectively.

Afterwards, the AMB system and the exosystem can be formulated in the following output regulation problem form,

$$
\begin{aligned}
\dot{\zeta}(t) & =\mathbf{A} \zeta(t)+\mathbf{B} i_{c}(t-\tau)+\sum_{j=1}^{s} \mathbf{P}_{j} w_{j}(t), \\
\dot{w}_{j}(t) & =\mathbf{S}(t) w_{j}(t), \text { for } j=1,2, \cdots, s . \\
e_{f}(t) & =K_{x} C_{\mathrm{amb}} \zeta(t)+K_{i} i_{c}(t-\tau) .
\end{aligned}
$$

Based on the validated AMB system model, the rotor levitation controller and the 


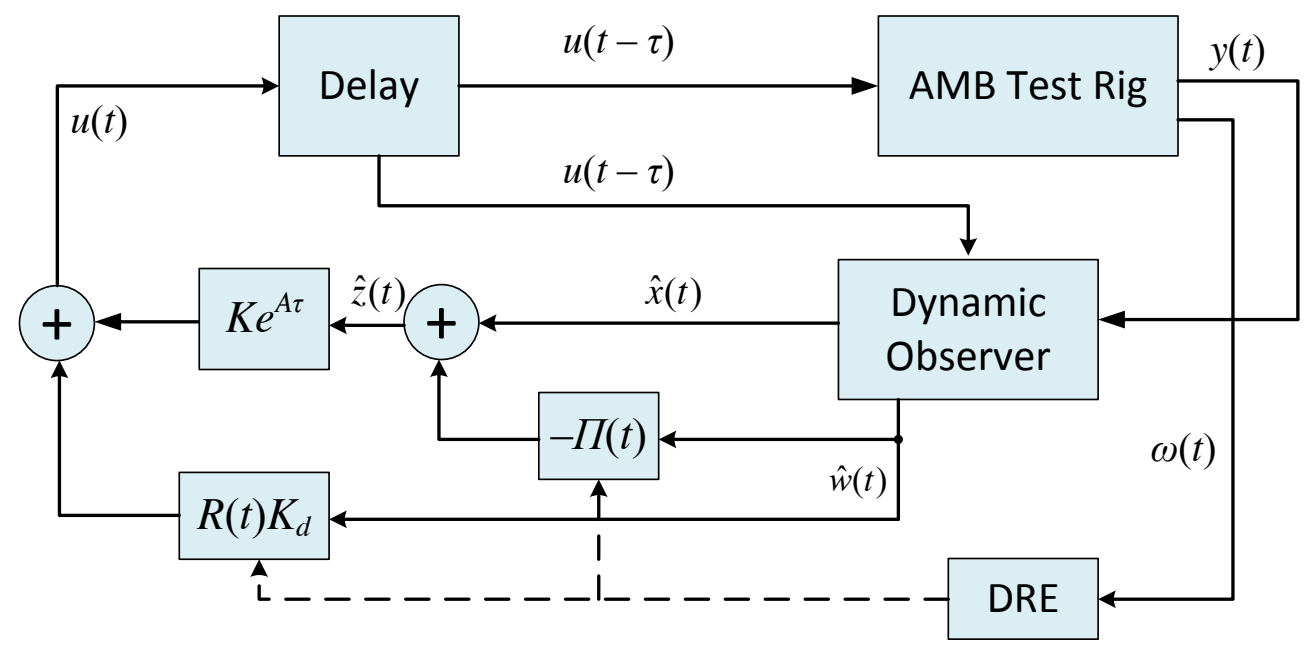

Figure 6.2: Block diagram of the controller implementation under time-varying rotational speed.

differential regulator based unbalance compensation control law are designed. The leviation controller is based on the TPF control law for the undisturbed case $(\omega \equiv 0)$, which is detailed in Section 5.1.2 with $\bar{D}_{0}=2.02 \times 10^{-4} \mathrm{~s}$. The results of Lemma 4.3.2 yield a dynamic observer to estimate the states of the plant and the exosystem at different rotational speeds. The observer satisfies $\left\|\phi_{\Xi^{L}}\left(t, t_{0}\right)\right\| \leq k_{L} e^{-l_{0}\left(t-t_{0}\right)}$ for $k_{L}>0, l_{0}>0$ and $\Xi^{L}(t)$ is the system matrix of the estimation error. The designed observer is utilized to construct the output feedback controller and the implementation of the final controller is illustrated in Fig. 6.2.

\subsubsection{Simulation results}

The proposed method is verified by simulation for autobalancing at both varying rotational speeds and constant speeds on the model of the flexible rotor AMB test rig. A constant input delay (5.4) is introduced to the simulation, with $D=2.02 \times 10^{-4} \mathrm{~s}$. Since AMB systems are of non-minimum phase, a control gain $K_{r}$ is designed to stabilize the unbounded compensator gains and the unified gradient method described in Section 4.3.3 is applied to ensure that $\inf _{K_{r}} J_{1}\left(K_{r}\right)$ is achieved.

The eigenvalues corresponding to the desired pole locations are composed of the originally 

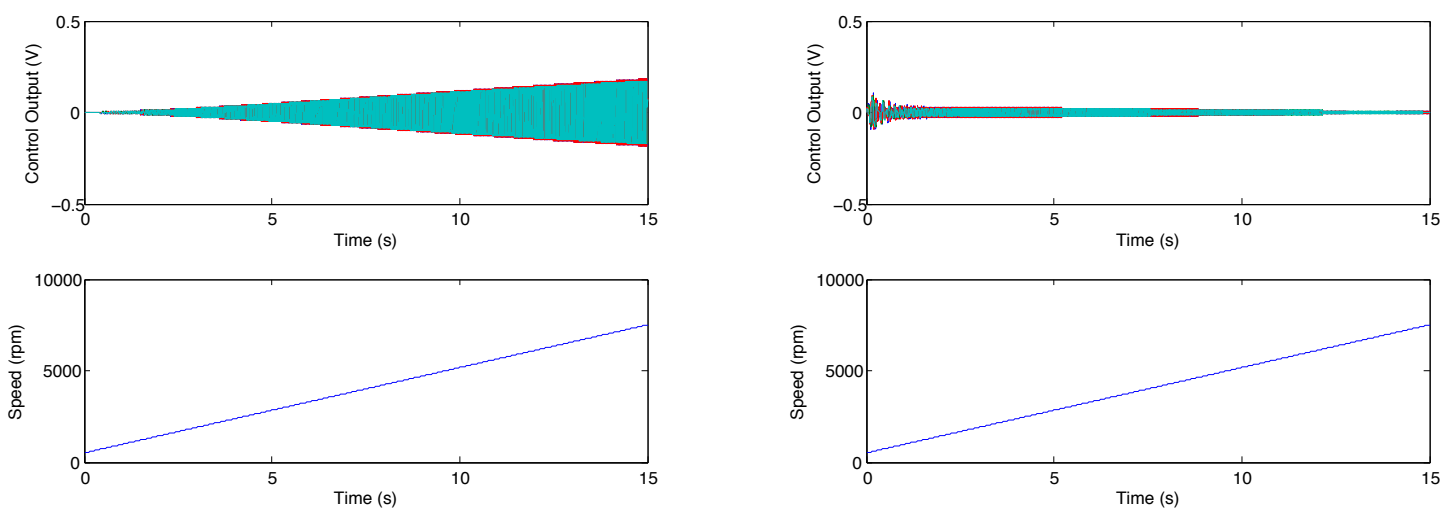

Figure 6.3: Simulated control voltages without (left) and with (right) the differential regulator as the rotating speed varies from 1,000 to $7,500 \mathrm{rpm}$ using state feedback.
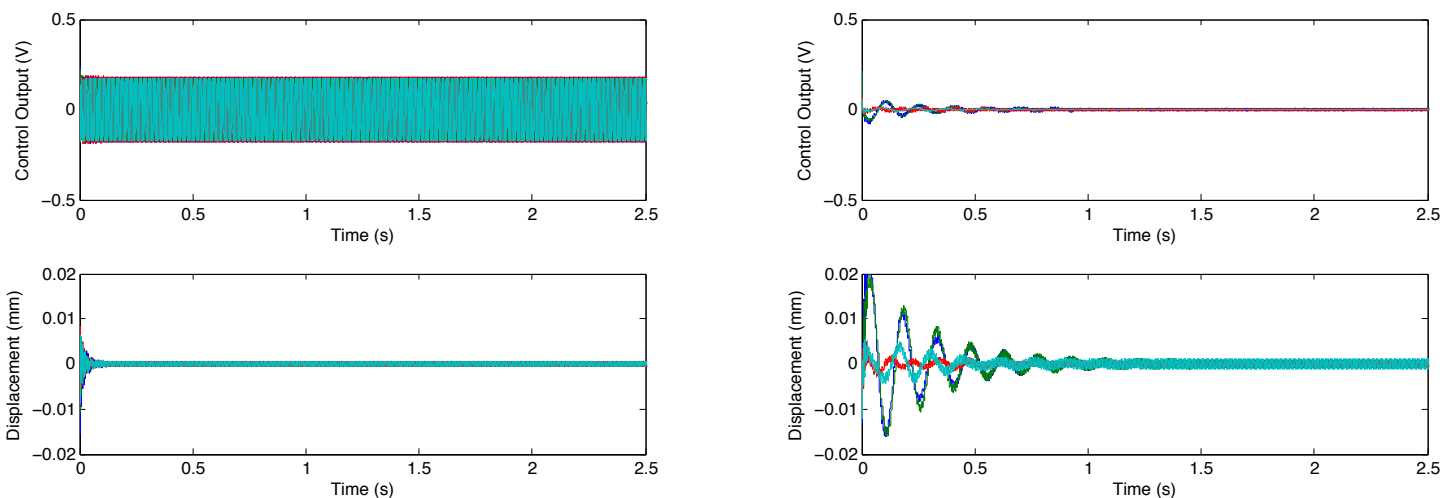

Figure 6.4: Simulated control voltages and rotor displacements without (left) and with (right) the differential regulator at 7,500 rpm using state feedback.

stable eigenvalues and the originally anti-stable eigenvalues with their real parts multiplying by -1 . The free parameter $U$ is initially selected as a matrix with all the components equal to 1 . After 117 iterations through the unified gradient method, $\frac{\partial J_{1}}{\partial U}$ approaches 0 , and $J_{1}=1.83 \times 10^{-4}$. The corresponding $K_{r}$ is applied to the differential regulator equation to generate the compensator gains that locally minimize the residual error in the regulated AMB control voltage.

Shown in Fig. 6.3 are the control voltages of the four radial axes as the rotational speed varies from 1,000 to 7,500 rpm using state feedback, without and with the differential regulator, 

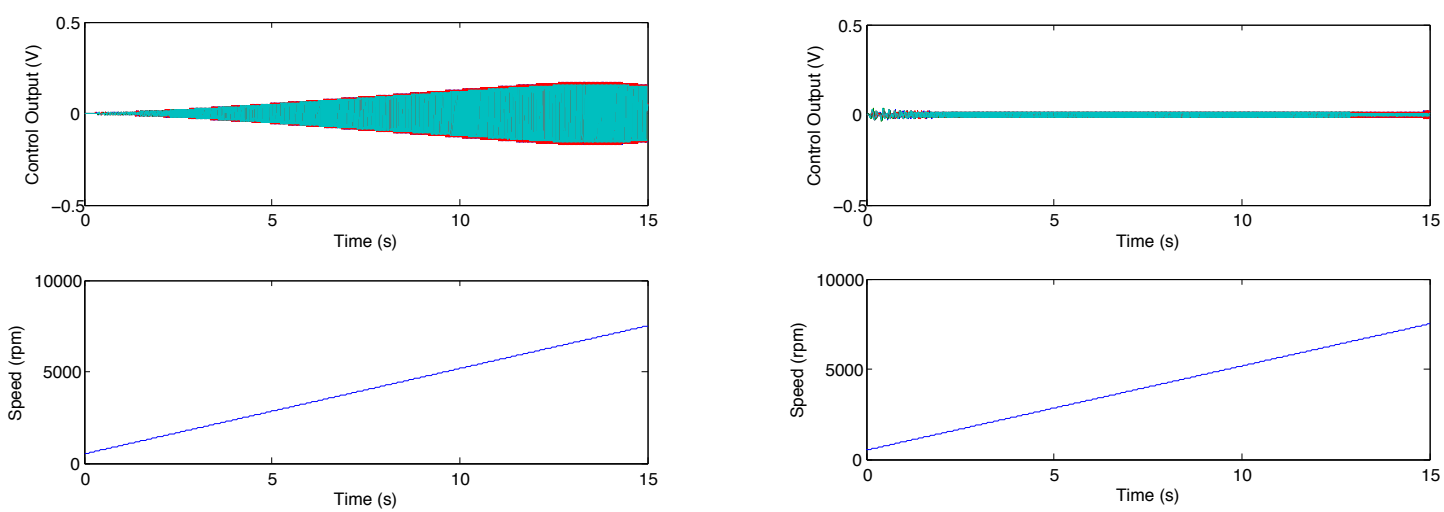

Figure 6.5: Simulated control voltages without (left) and with (right) the differential regulator as the rotating speed varies from 1,000 to $7,500 \mathrm{rpm}$ using output feedback.
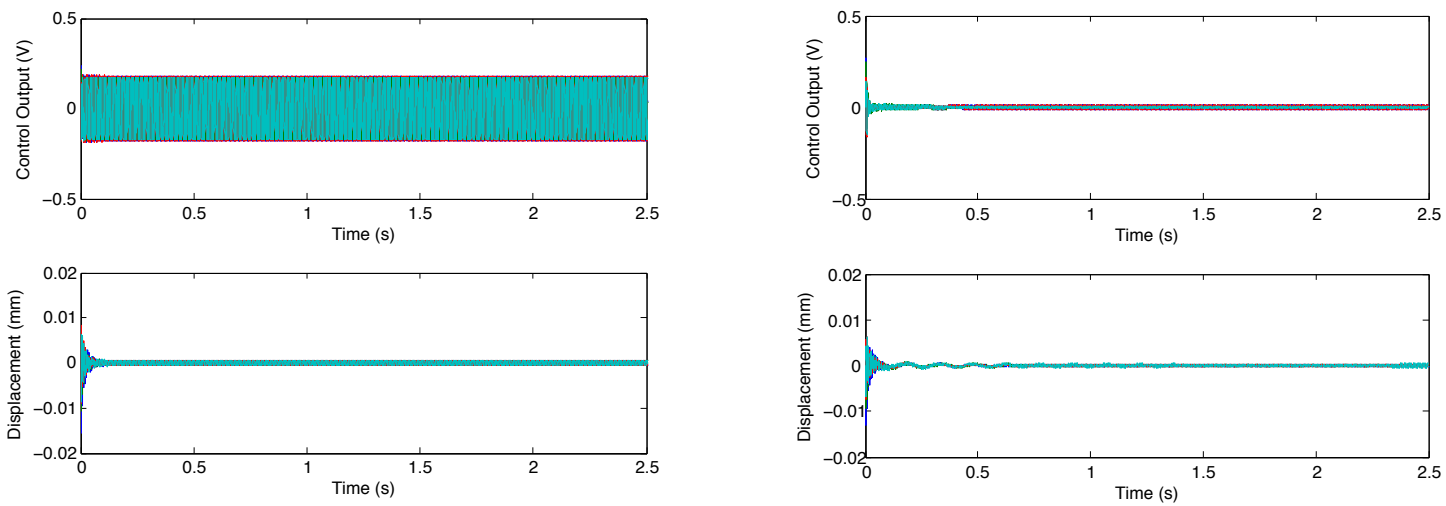

Figure 6.6: Simulated control voltages and rotor displacements without (left) and with (right) the differential regulator at 7,500 rpm using output feedback.

respectively. It can be observed that the control voltage is significantly reduced over the wide speed range. Fig. 6.4 shows the simulated control voltages and rotor displacements without and with the differential regulator at 7,500 rpm using state feedback. The vibration levels under both cases are similar while the control voltage is significantly reduced with the differential regulator.

Shown in Fig. 6.5 are the control voltages of the four radial axes as the rotating speed increases from 1,000 to 7,500 rpm using output feedback, without and with the differential regulator, respectively. Similar to the state feedback case, the control voltage is significantly 
reduced and almost remains steady as the rotational speed increases. Fig. 6.6 shows the simulated control voltages and rotor displacements without and with the differential regulator at 7,500 rpm using output feedback. Compared to the results without the differential regulator, the vibration level is noticeably smaller and the control voltage is significantly reduced with the proposed differential regulator.

To better illustrate the effectiveness of the differential regulator based output regulation method, Fig. 6.7 shows the 3-dimensional plot of the control voltage and the rotor displacement with the differential regulator switched off and on at a fixed rotational speed. The differential regulator is activated after 2 seconds, and the control outputs quickly converges to much smaller values at both driven and non-driven ends while the rotor displacements are also noticeably reduced, which indicates that the rotational axis closely approaches the principle axis of inertia and the AMB system achieves autobalancing.

It is observed in the simulation that the proposed differential regulator based output regulation approach has effectively achieved autobalancing of an AMB system for varying rotational speeds. Because of the computation and hardware limitations, currently it is difficult to implement the algorithms on the prospective platform. In order to conduct experiments to better demonstrate the strength and potential of the proposed method, another test rig is adopted.

\subsection{Unbalance suppression on a balancing beam}

The experimental studies have been conducted on a balancing beam (as shown in Fig. 6.8) to validate the unbalance suppression capability of the differential regulator based output regulation method. The test rig consists of two sets of active electromagnets at each end of a pivoting beam. The magnetic coils generate electromagnetic forces to keep the beam near the center pivot point. The test rig has two rails to prevent the beam from damaging the coils, which limit the range of angular motion of the beam to $\pm 0.013 \mathrm{rad}$. The angle of 


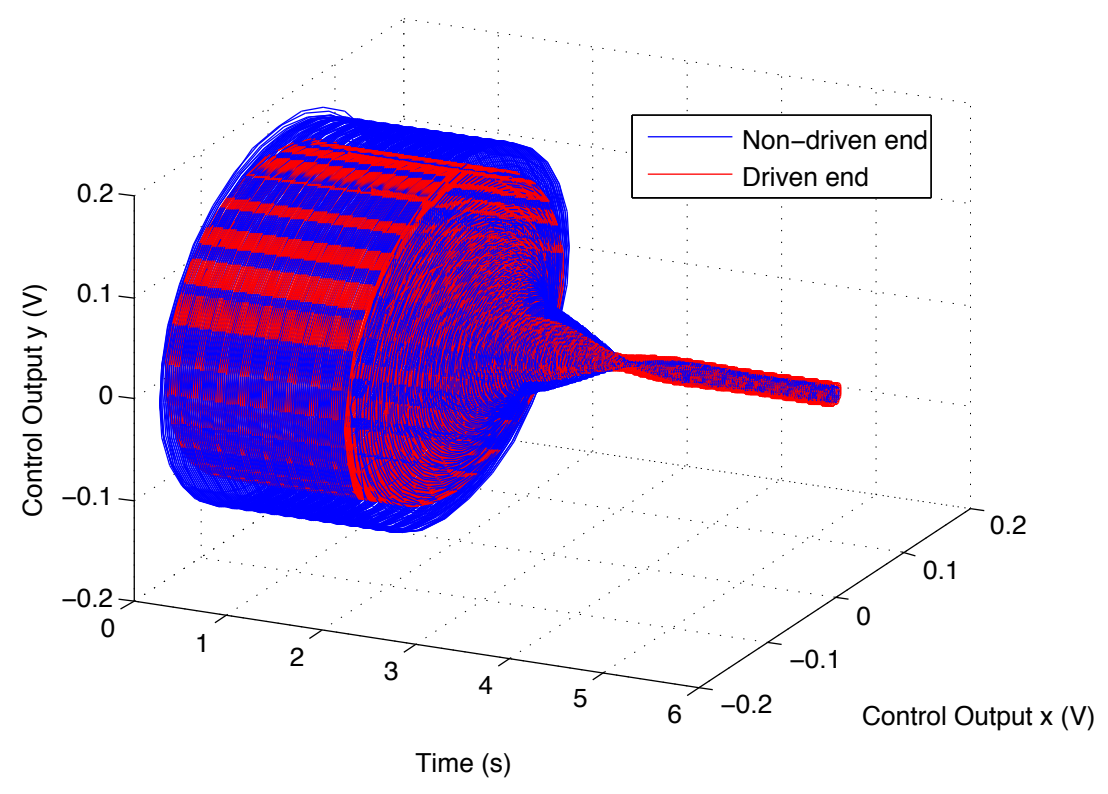

(a) Control voltages.

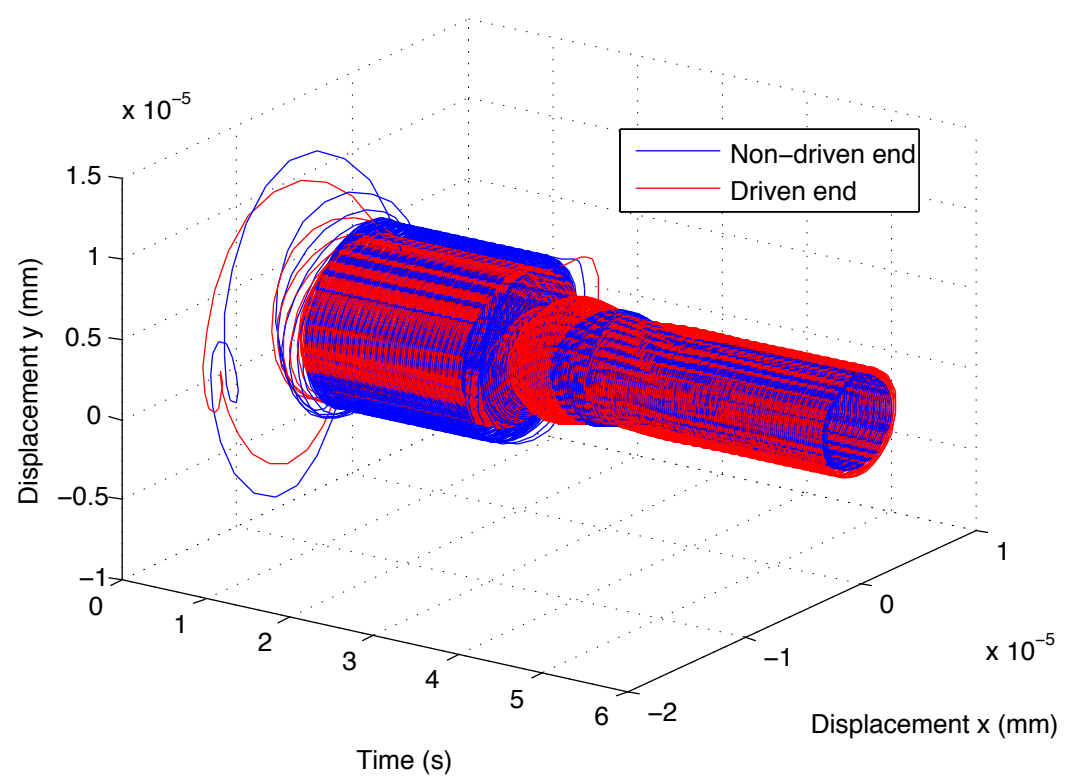

(b) Rotor displacements.

Figure 6.7: Simulated control voltages and rotor displacements with the differential regulator turned on at 2 seconds.

the beam with respect to the fixed base is measured by two proximity sensors. A brushless DC motor, which currently can run up to 1,200 rpm, is mounted on a rack attached to the 


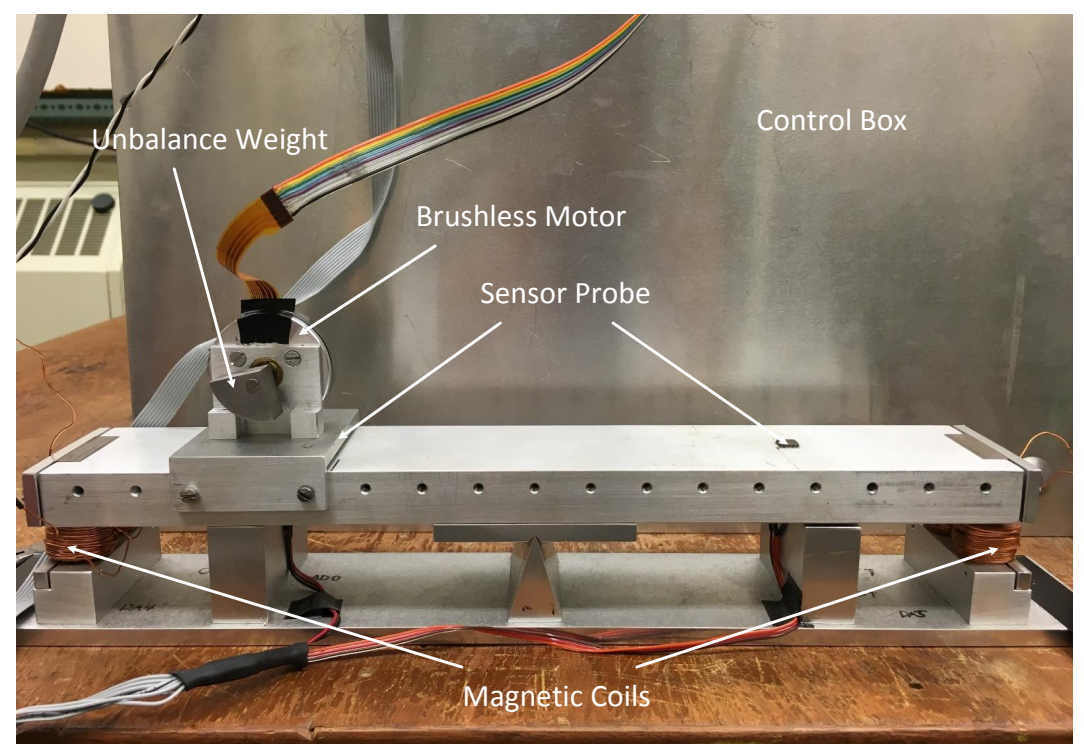

Figure 6.8: The balancing beam test rig.

beam with an unbalance weight, and when the motor starts rotating, the unbalance weight injects significant disturbance to the system. The control box includes the voltage regulators, sensor conditioning and measurement circuits, motor speed controllers. A Linux computer running an open source real-time control software called Scicos-Lab is used to process the sensor measurements, implement the control system design and feed the control signal to the test rig.

The controller design is based on the dynamic model of the test rig. The balancing beam includes two states, the beam angle and the angular velocity, while the input and output are the control current and the sensor probe readings, respectively. The dynamics of the beam can be modeled as follows,

$$
J_{b} \ddot{\theta}=-D_{b} \dot{\theta}+T_{2}-T_{1}
$$

where $\theta$ is the angle between the beam and the horizon, $T_{1}$ and $T_{2}$ are torques provided by the two active electromagnets and the total torque $T=T_{2}-T_{1} . J_{b}$ is the moment of inertial of the beam and $D_{b}$ is the internal damping. 
The following differential equations are used to model the two active electromagnets:

$$
\begin{aligned}
& L_{1} \dot{I}_{1}=v_{1}-I_{1} \dot{L}_{1}-R_{1} I_{1}, \\
& L_{2} \dot{I}_{2}=v_{2}-I_{2} \dot{L}_{2}-R_{2} I_{2},
\end{aligned}
$$

where $L_{1}=\frac{L_{0} g_{0}}{g_{0}+\theta}$ and $L_{2}=\frac{L_{0} g_{0}}{g_{0}-\theta}, I_{1}$ and $I_{2}$ are the final control currents to the coils, $I_{1}=I_{b}+I$, $I_{2}=I_{b}-I, I_{b}$ is the bias current and $I$ is the perturbation control current, $L_{0}$ is the inductance of the coil when $\theta=0$ and $g_{0}$ is the maximum angle when the beam reaches one of the rails. The torques are calculated as follows

$$
\begin{aligned}
& T_{1}=c_{t}\left(\frac{g_{0} I_{1}}{g_{0}+\theta}\right)^{2}, \\
& T_{2}=c_{t}\left(\frac{g_{0} I_{2}}{g_{0}-\theta}\right)^{2},
\end{aligned}
$$

where $c_{t}$ is a constant based on the air gap and flux density.

The brushless motor with the unbalance weight is modeled as the exosystem. The motor generates a sinusoidal torque $T_{d}$ caused by the centripetal force to keep the unbalance on its orbit. In addition, the motor mass is added to the beam so the moment of inertia $J_{b}$ is increased and the mass induces a constant torque $T_{m}$. The parameters $T_{d}, J_{b}$ and $T_{m}$ can be approximated as follows,

$$
\begin{aligned}
& T_{d}=m_{d} \omega^{2} r_{d} l_{d} \sin (\omega t), \\
& J_{m}=m_{m} l_{m}^{2}, \\
& T_{m}=m_{m} g l_{m},
\end{aligned}
$$

where $m_{d}$ is the mass of the unbalance, $r_{d}$ is the radius of the orbit of the unbalance weight center gravity around the rotor, $l_{d}$ is the projection of the length between the motor shaft and the pivot onto the horizon, $\omega$ is the motor speed, $m_{m}$ is the motor mass, $l_{m}$ is the distance 


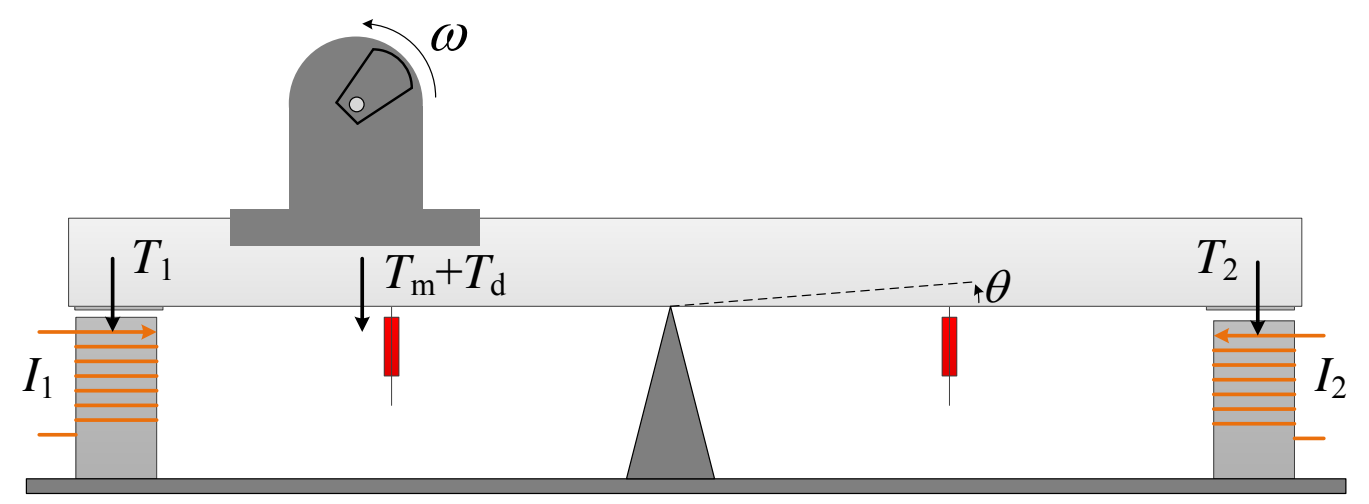

Figure 6.9: Illustrative diagram of the balancing beam test rig.

between the motor and the pivot, $g$ is the acceleration of gravity.

A diagram illustrating the basic function of the test rig is shown in Fig. 6.9. The linearized plant subject to input delay and the exosystem can be grouped in the following output regulation form

$$
\begin{aligned}
\dot{x}(t) & =A x(t)+B I(t-\tau)+P w(t), \\
\dot{w}(t) & =S(t) w(t) \\
e(t) & =C x(t)
\end{aligned}
$$

where

$$
\begin{aligned}
& x(t)=\left[\begin{array}{c}
\theta(t) \\
\dot{\theta}(t)
\end{array}\right], \quad A=\left[\begin{array}{cc}
0 & 1 \\
\frac{4 c_{t} I_{b}^{2}}{J_{b} g_{0}} & -\frac{D_{b}}{J_{b}}
\end{array}\right], \quad B=\left[\begin{array}{c}
0 \\
-\frac{4 c_{t} I_{b}}{J_{b}}
\end{array}\right], \\
& S(t)=\left[\begin{array}{cc}
0 & \omega(t) \\
-\omega(t) & 0
\end{array}\right], \quad P=\left[\begin{array}{cc}
0 & 0 \\
\frac{1}{J_{b}} & 0
\end{array}\right], \quad C=\left[\begin{array}{ll}
1 & 0
\end{array}\right] .
\end{aligned}
$$

The exosystem is derived based on the unbalance force equation. Since it is not appropriate to apply autobalancing to the balancing beam, suppression of the unbalance disturbance 
becomes the primary objective to achieve and the error to be regulated is the tilting angle of the beam under different motor speeds. Besides, a constant delay $\tau$ is added at the output of the controller to simulate the input delay. The LMI approach presented in Section 5.1 is applied to synthesize the TPF controller with the maximum delay bound, where the corresponding maximum input delay $\bar{D}=1.54 \mathrm{~ms}$. Similar to Section 6.1 , the results of Lemma 4.3.2 are adopted to construct an observer to estimate the states of the plant and the exosystem at different rotational speeds. The state observer is utilized to formulate the output feedback controller, with a structure similar to Fig. 6.2.

\subsubsection{Simulation results}

The proposed differential regulator based output regulation method is first verified by simulation for unbalance suppression at both constant motor speed and varying motor speeds on the model of the balancing beam test rig. Shown in Fig. 6.10 are the control current and beam tilting angle without the differential regulator when the motor speed stays at 980 rpm while the differential regulator is activated in Fig. 6.11. The red dashed line indicates the mechanical boundary created by the two rails. It can be observed that the tilting angle quickly converges to near zero and the control current is notably reduced.

Shown in Fig. 6.12 are the control current and beam tilting angle without the differential regulator when the motor speed increases from 0 to 1,200 rpm while the differential regulator is activated in Fig. 6.13. It can be observed that the tilting angle remains near zero for the wide motor speed range and the control current is notably reduced. In addition, when the differential regulator is activated, the angle clearance is significantly bigger at higher motor speed while the beam almost hits the rails and the system becomes unstable when the differential regulator is off. 

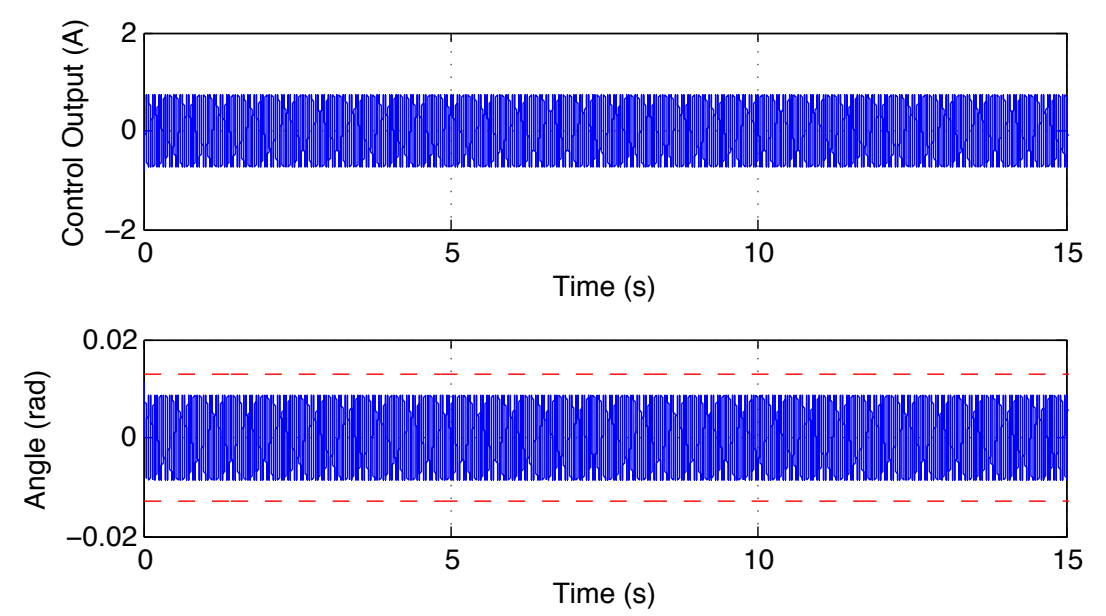

Figure 6.10: Simulated control current and titling angle without the differential regulator at $980 \mathrm{rpm}$.
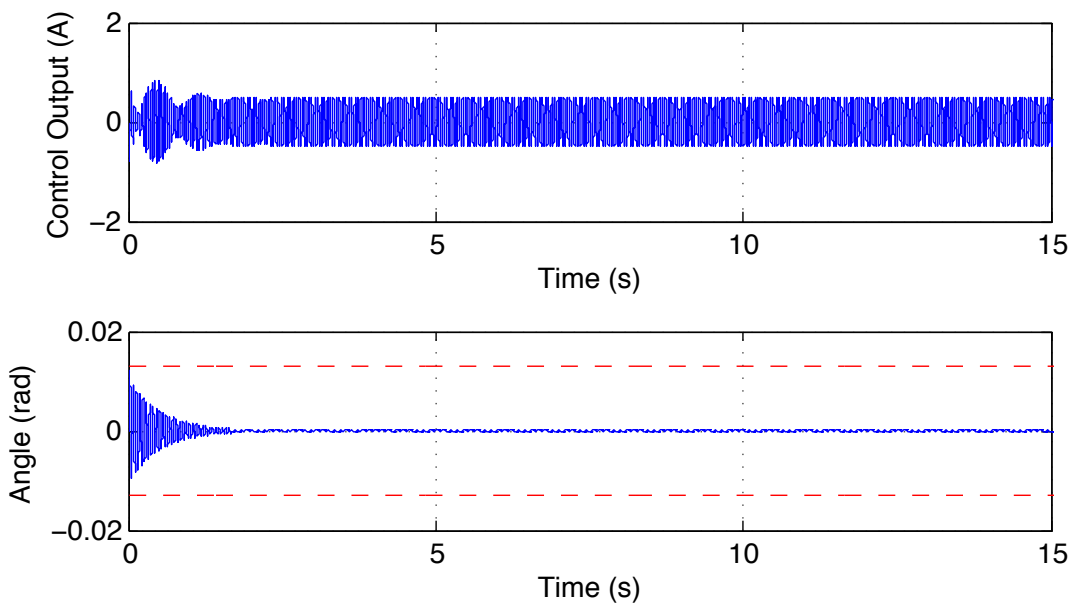

Figure 6.11: Simulated control current and titling angle with the differential regulator at 980 rpm.

\subsubsection{Experimental results}

The proposed controller is implemented on the balancing beam test rig. The algorithms are formulated and programmed on the open source real-time control platform Scicos-Lab. A constant delay $\tau=1.54 \mathrm{~ms}$ is added at the output of the controller to simulate the input delay and a TPF controller synthesized from the LMI optimization procedure is used for the 

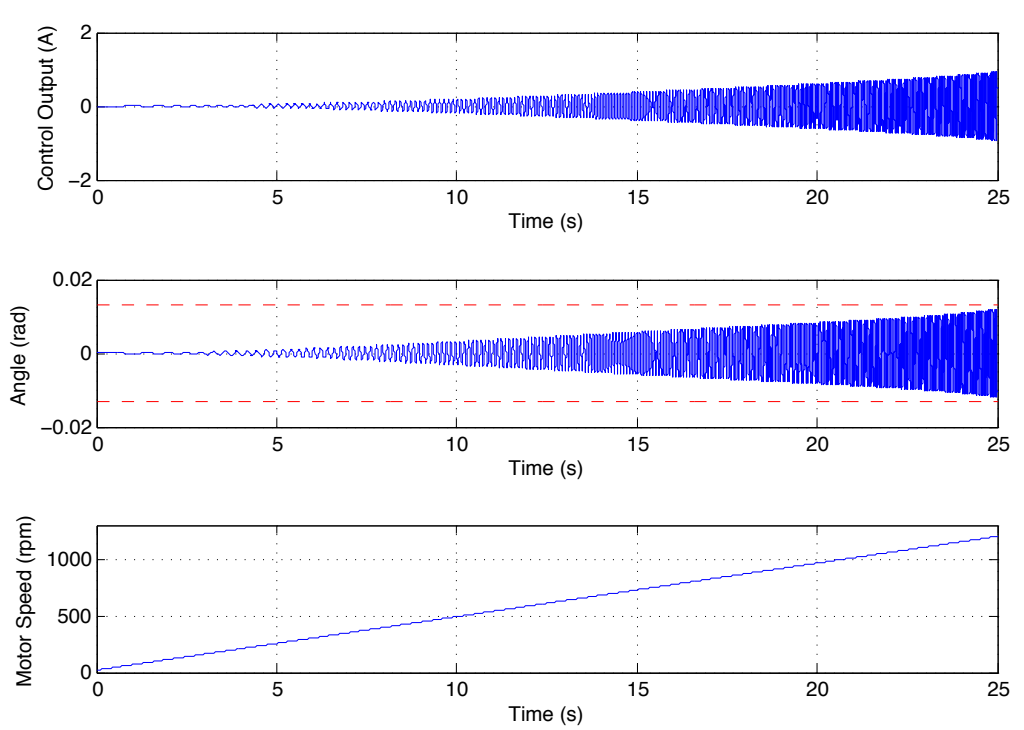

Figure 6.12: Simulated control current and titling angle without the differential regulator from 0 to $1,200 \mathrm{rpm}$.
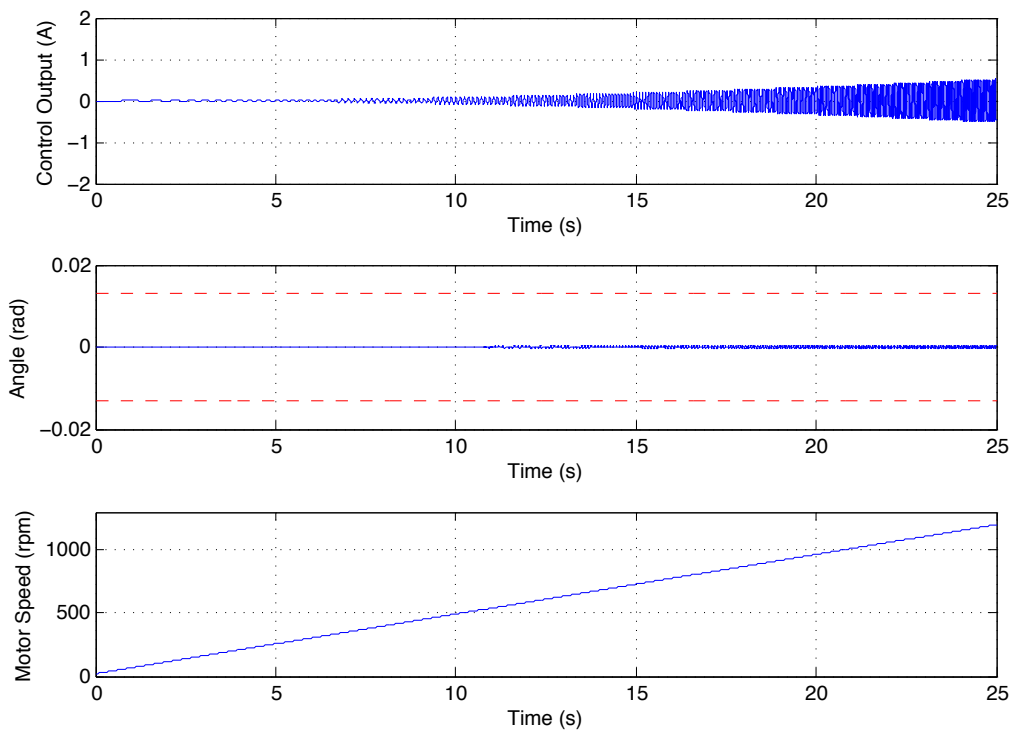

Figure 6.13: Simulated control current and titling angle with the differential regulator from 0 to $1,200 \mathrm{rpm}$.

levitation. The graphic user interface (GUI) in Scicos-Lab facilitates the testing of different control designs, and several key parameters such as the beam tilting angle, perturbation current are displayed on real-time plot windows. During experiments, those parameters can 
be saved in the computer for post-processing and frequency response analysis.

Shown in Fig 6.14 are the experimental results without and with the differential regulator when the motor runs at $685 \mathrm{rpm}$. The corresponding control currents, beam titling angles, and FFTs of the sensor measurements without and with the differential regulators are shown on the left and right columns, respectively. It is observed that with the regulator activated, the beam titling angles are reduced for more than 50\%, and the control current is also notably reduced. The FFT of the sensor measurements indicates that the synchronous vibration is fully rejected when the differential regulator is active.

Shown in Fig 6.15 are the experimental results without and with the differential regulator when the motor runs from 0 to $685 \mathrm{rpm}$ and then back to $0 \mathrm{rpm}$ within 30 seconds. The red curves indicate the results with the differential regulator activated while the blue curves indicate the results without the regulator. With the increasing of the motor speed, the unbalance forces become larger. It clearly shows that when the differential regulator is not active, in order to maintain the balance of the beam, the controller has to generate larger force to counteract the disturbance while the titling angle still increases significantly. When the differential regulator is active, the control output remains almost steady and the beam titling angles are also contained in a small level. The experimental results are consistent with the simulation results for both constant speed and time-varying speeds. The reason the speed is limited to near $700 \mathrm{rpm}$ is due to the open-source software limitation. Because of unknown software issue, the original analog ports can not support three analog inputs simultaneously, and the speed signal has to be imported through the digital port and utilized in the control signal calculation after filtering. However, when the motor reaches 1,200 rpm, the noise level prevents the rotating frequency from filtering out of the digital signal. The experimental results from 0 to near $700 \mathrm{rpm}$ have demonstrated the effectiveness of the proposed method, and since new sets of control hardware and software have been planned, the current limitations can be well addressed in the near future. 

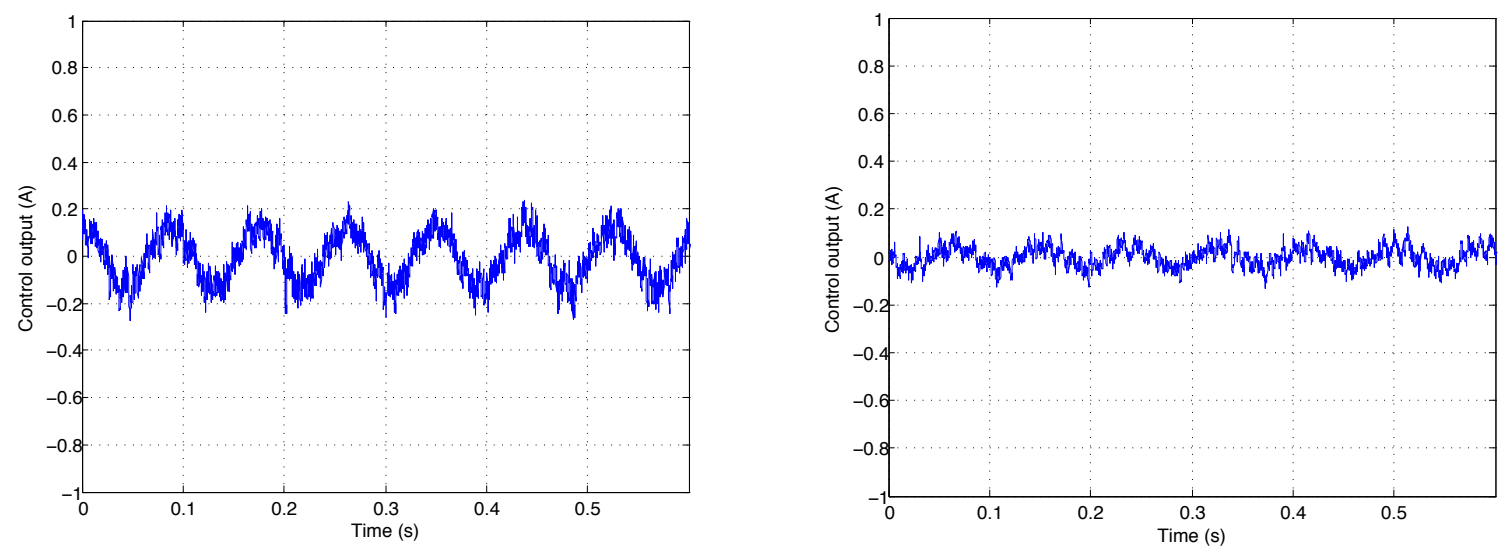

(a) Control currents.
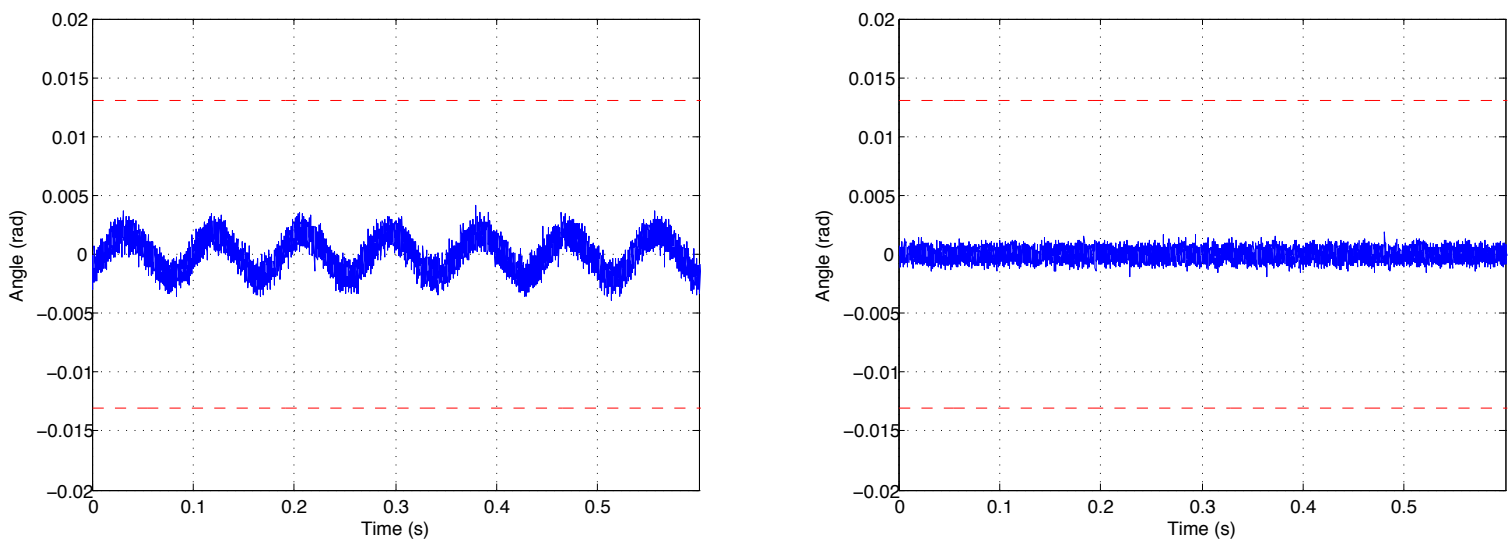

(b) Beam tilting angles.
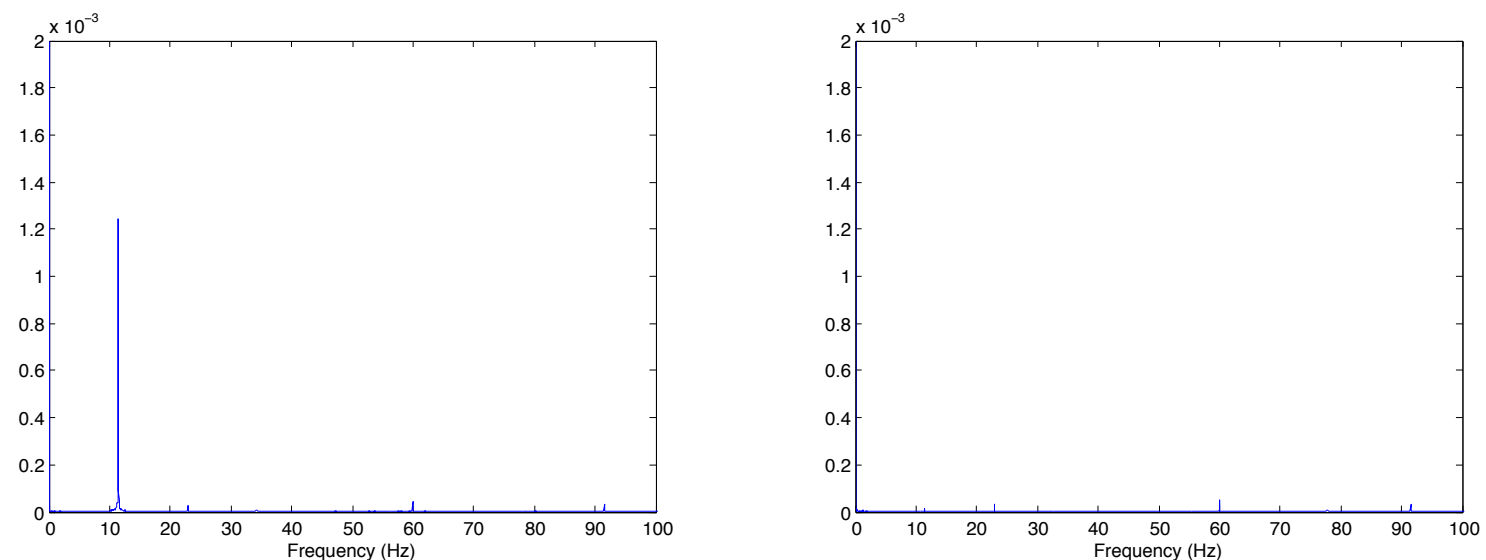

(c) FFT of the sensor measurements.

Figure 6.14: Experimental performance without (left column) and with (right column) the differential regulator at $685 \mathrm{rpm}$. 


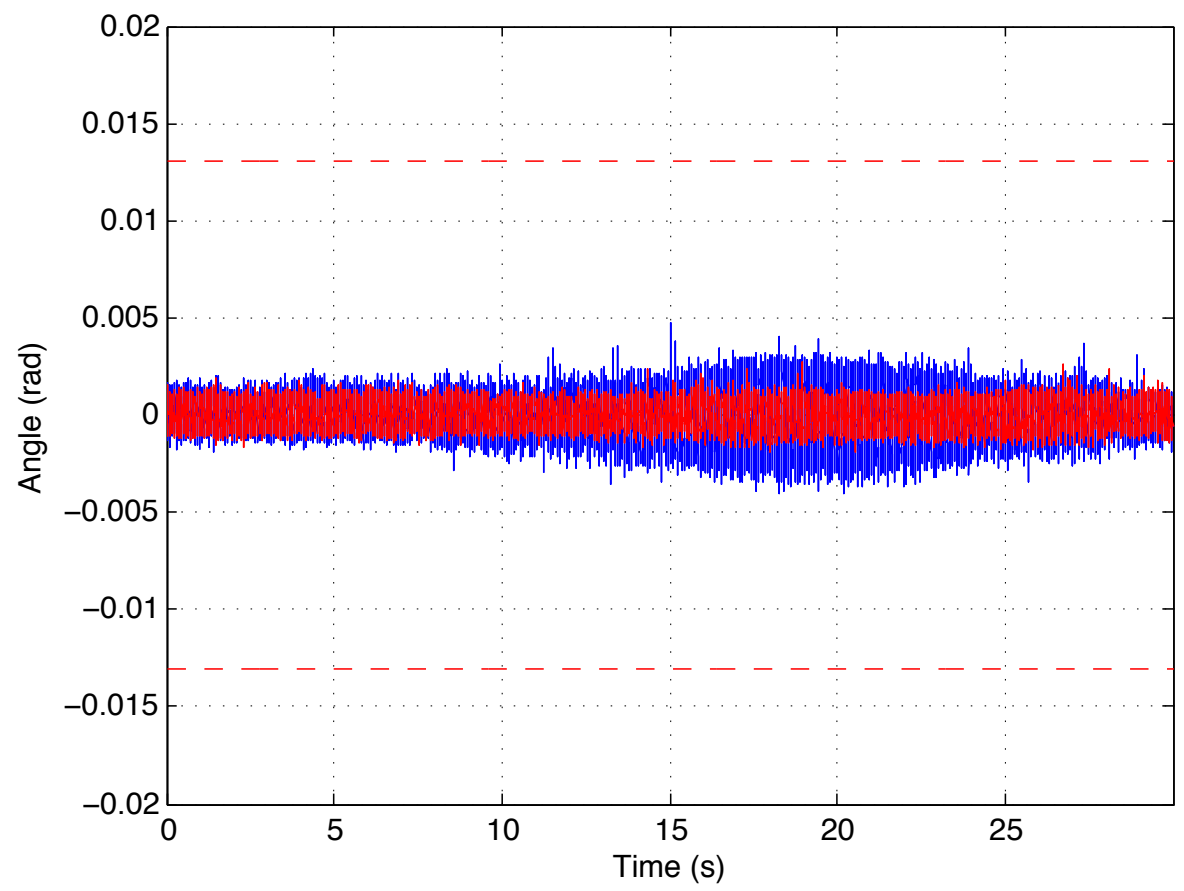

(a) Beam tilting angles.

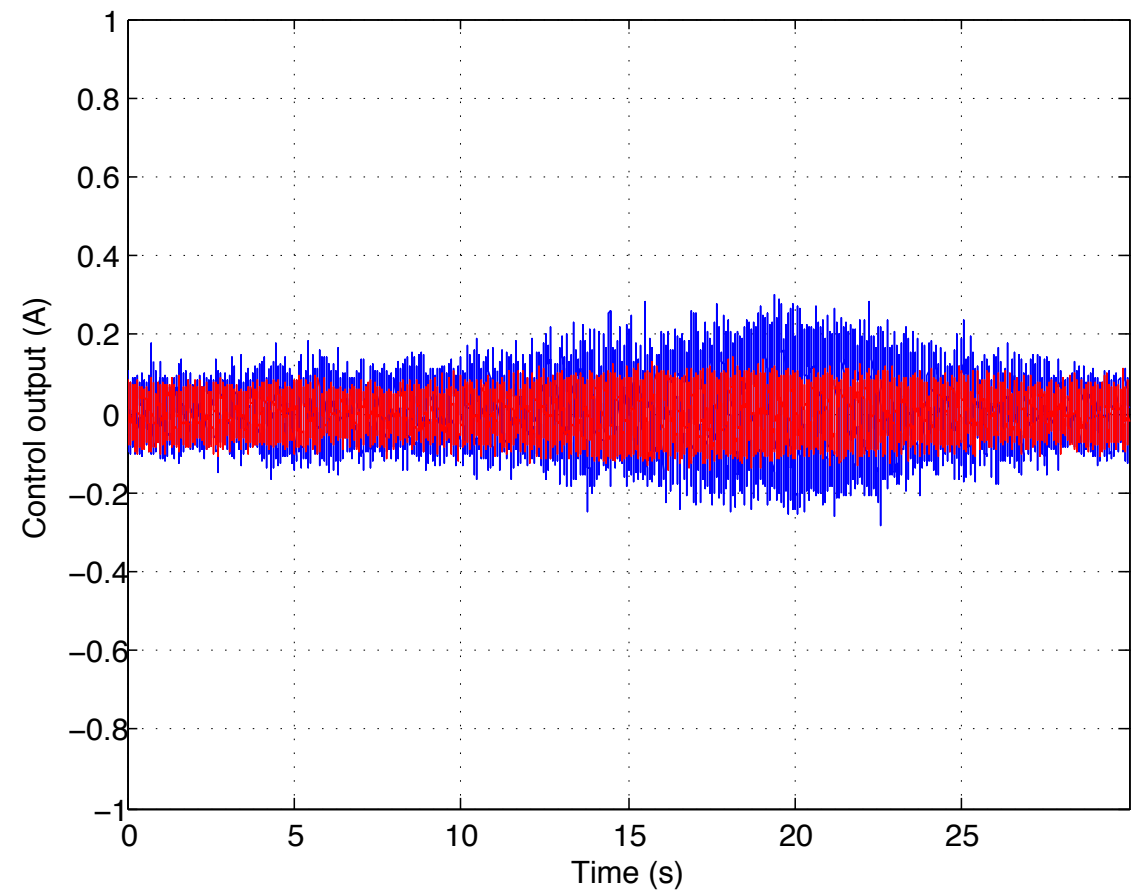

(b) Control currents.

Figure 6.15: Experimental performance without (blue curves) and with (red curves) the differential regulator from 0 to $685 \mathrm{rpm}$ and then back to $0 \mathrm{rpm}$. 


\subsection{Summary}

This chapter focuses on unbalance compensation of AMB systems subject to input delay with time-varying rotational speed. The differential regulator based output regulation method is first applied to the model of the flexible rotor AMB test rig for autobalancing at different rotational speed conditions, which has been verified in the simulation results. Then a balancing beam test rig is adopted to further validate the proposed compensation method. Extensive simulation and experimental results under time-varying rotational speed show close agreements and they have demonstrated the strength and potential of the proposed method. 


\section{Chapter 7}

\section{Conclusions}

\section{$7.1 \quad$ Summary}

This dissertation presents a systematic approach for the unbalance compensation of AMB systems with input delay. The potential applications of AMB supported compressors for offshore oil and gas production entail the system to be remotely located in severe environments for long operating hours. Since these areas are not easily accessible for frequent diagnosis and maintenance, AMB systems need to operate stably and robustly. Two main challenges of the applications have been addressed in this work. The first one is the time delay caused by long cables connecting the AMB control unit to the AMB actuators. Because time delay may degrade the system performance and even jeopardize the closed-loop system stability, the control of AMB systems subject to input delay effects has to be well addressed. Besides time delay, the unbalance disturbance is the other challenge for such applications. Considering any newly machined rotor always carries some small residual weight, unbalance effects cannot be ignored. If they are not suppressed properly, the rotor can be induced into whirling motion and the AMB system can become unstable. To satisfy the control requirements of a remotely located compressor supported by AMBs, a special advanced control method has been developed to contain the time delay effect and suppress the unbalance disturbance under 
different rotational speed conditions.

To first achieve stable rotor levitation with input delay effects, the TPF control method is applied. The controller corresponding to the maximal input delay the closed-loop system can tolerate is obtained iteratively from the solution of a local LMI problem. The ROMAC flexible rotor AMB test rig is used for experimental validation of the developed TPF control law. The TPF controller notably outperforms a previously designed $\mu$-synthesis robust controller when longer input delay is added and the rotor vibration level is sufficiently contained during the unbalance response test. Additionally, to reduce the rotor unbalance disturbance on AMB supported high speed rotating machineries subject to input delay, with the TPF control law serving as the feedback controller, an unbalance compensation method based on the solution of an equivalent output regulation problem at a constant rotational speed is developed. By applying the output regulation mechanism to AMB systems for unbalance reduction, the unbalance force is modeled as a time-invariant exosystem and two output regulation scenarios are investigated that define the control forces and rotor displacements as the errors to be regulated. Both simulation and experimental results reveal that the synchronous rotor vibrations and the magnitude of the AMB control currents can be significantly reduced using the developed output regulation compensator.

After considering unbalance compensation for input-delay AMB systems under a constant rotational speed, the research extends to the autobalancing and unbalance suppression problem of AMB systems subject to input delay at varying rotational speed. A differential regulator based output regulation approach is presented to first address the autobalancing problem of AMB systems for varying rotational speed cases. When the rotational speed dynamically changes, the exosystem derived from the unbalance forces becomes time-varying. After formulating the output regulation problem with a time-varying exosystem, it is observed that the compensator gains can be obtained based on the solution of the DRE and a sub-optimal solution is developed using the unified gradient method for non-minimum phase systems so that the regulator gains are always bounded and the residual error in the regulated AMB 
control force is locally minimized. Simulation results are presented to verify the effectiveness of the method. Lastly, a balancing beam test rig is adopted to further validate the proposed compensation approach under time-varying rotational speed and the beam titling angle is defined as the regulation error. Both simulation and experimental results demonstrate the strength and potential of the developed method.

Based on what has been completed in the dissertation, the most significant novelties and contributions are summarized as follows: given that the unbalance compensation of AMB systems subject to time delay has not been documented in the previous literature, this dissertation has developed a truncated predictor feedback based output regulation mechanism to control AMB supported high speed compressors in remotely operated applications, and considering that little literature has addressed the time-varying exosystem problem and the current approach has restrictions, this dissertation has derived a practical differential regulator based output regulation algorithm to achieve locally minimized residual error in the regulated output for non-minimum phase systems, such as AMB systems, that are affected by the disturbance generated from time-varying exosystems.

\subsection{Future work}

Following the work completed in this dissertation, there are still several problems to be addressed in the future:

1. The size of the plant model plays a critical role in the differential regulator based output regulation approach. For complex dynamic systems which are represented by high order plant models, the corresponding compensator will have high orders as well. For implementation purpose, this may place an excessive demand on the computing power from the hardware, which sometimes may become impractical. Therefore, a systematic approach can be developed to reducing the size of the developed compensator while maintaining its appealing features. 
2. The unified gradient method is adopted to locally minimize the residual error in the regulated output. Additional investigation can be taken to verify if the residual error can be further reduced using other approaches.

3. Since the current problem formulation only considers a time-invariant plant subject to input delay, there might be new findings if the current framework is extended to a time-varying plant.

4. Uncertainties may be considered in the development of the compensator to enhance the robustness of the output regulation problem for a system with time delay. 


\section{Publications}

1. L. Di, C. Y. Chen, C. H. Lin, and Z. Lin, "Characteristic Model Based All-Coefficient Adaptive Control of a High-Speed Desorption Pump Supported by AMBs", in Proceedings of the 15th International Symposium on Magnetic Bearings (ISMB15), August 3-6, 2016, Kitakyushu, Japan.

2. L. Di, S. Y. Yoon, and Z. Lin, "Autobalancing of AMB Systems Using a Differential Regulator Based Output Regulation Approach", in Proceedings of the 15th International Symposium on Magnetic Bearings (ISMB15), August 3-6, 2016, Kitakyushu, Japan.

3. S. Y. Yoon, L. Di, and Z. Lin, "An Output Regulation Approach to Rotor Autobalancing in Active Magnetic Bearing Systems with Input Delay", in Proceedings of the IEEE International Conference on Advanced Intelligent Mechatronics, July 12-16, 2016, Banff, Canada.

4. L. Di and Z. Lin, "Control of a Flexible Rotor Active Magnetic Bearing Test Rig: A Characteristic Model Based All-Coefficient Adaptive Control Approach", Control Theory and Technology, Volume 12, Issue 1, page 1-12, 2014.

5. S. Y. Yoon, L. Di, and Z. Lin, "Unbalance compensation for AMB systems with input delay: an output regulation approach", Control Engineering Practice (CEP), Volume 46, Pages 166-175, January 2016. 
6. S. Y. Yoon, L. Di, P. Anantachaisilp, and Z. Lin, "Truncated Predictor Feedback Control for Active Magnetic Bearing Systems With Input Delay", IEEE Transactions on Control Systems Technology, vol.PP, no.99, page 1-8, March 2016.

7. X. Lyu, L. Di, S. Y. Yoon, Z. Lin, and Y. Hu, "A platform for analysis and control design: Emulation of energy storage flywheels on a rotor-AMB test rig", Mechatronics, Volume 33, Pages 146-160, February 2016.

8. J. Zhou, L. Di, C. Cheng, Y. Xu, and Z. Lin, "A Rotor Unbalance Response Based Approach to the Identification of the Closed-loop Stiffness and Damping Coefficients of Active Magnetic Bearings", Mechanical Systems and Signal Processing (MSSP), Volumes 6667, Pages 665678, January 2016.

9. L. Di, J. Zhou, C. Cheng, and Z. Lin, "Experimental Verification of a Rotor Unbalance Response Based Approach to the Identification of Magnetic Bearing Support Parameters", in Proceedings of the 14th International Symposium on Magnetic Bearings (ISMB14), Aug. 11-14, 2014, Linz, Austria.

10. P. Anantachaisilp, L. Di, S. Y. Yoon, and Z. Lin, "Control of Active Magnetic Bearing Systems with Input Delay for Applications in Remotely Controlled Turbomachinery", in Proceedings of the 53rd IEEE Conference on Decision and Control (CDC), Dec. 15-17, 2014, Los Angeles, California.

11. X. Lyu, L. Di, S. Y. Yoon, Z. Lin, and Y. Hu, "Emulation of Energy Storage Flywheels on a Rotor-AMB Test Rig", in Proceedings of the 14th International Symposium on Magnetic Bearings (ISMB14), Aug. 11-14, 2014, Linz, Austria.

12. L. Di, S. E. Mushi, Z. Lin, and P. E. Allaire, "Characteristic Model based All-coefficient Adaptive Control of a Flexible Rotor Suspended on Active Magnetic Bearings", in Proceedings of the 13th International Symposium on Magnetic Bearings (ISMB13), August 6-9, 2012, Arlington, Virginia. 


\section{Bibliography}

[1] G. Schweitzer and E. Maslen, Eds., Magnetic Bearings. Springer, 2009.

[2] http://www.wildpackets.com/resources/compendium/ethernet/propagation_delay. Retrieved 2010-11-09.

[3] Z. Lin and H. Fang, "On asymptotic stability of linear systems with delayed input," IEEE Trans. Autom. Control, vol. 52, pp. 998-1013, 2007.

[4] B. Zhou, Z. Lin, and G. R. Duan, "Truncated predictor feedback for linear systems with long time-varying input delay," Automatica, vol. 48, pp. 2387-2399, 2012.

[5] S. E. Mushi, Robust Control of Rotordynamic Instability in Rotating Machinery Supported by Active Magnetic Bearings, PhD Dissertation, University of Virginia, USA, 2012.

[6] L. Di and Z. Lin, "Control of a flexible rotor active magnetic bearing test rig: A characteristic model based all-coefficient adaptive control approach," Control Theory and Technology, vol. 12, pp. 1-12, 2014.

[7] K. Gu and S. Niculescu, "Survey on recent results in the stability and control of timedelay systems," J. Dyn. Sys., Meas., Control, vol. 125, pp. 158-165, 2003.

[8] J. P. Richards, "Time-delay systems: an overview of some recent advances and open problems," Automatica, vol. 39, pp. 1667-1694, 2003.

[9] L. Dugard and E. I. Verriest, Eds., Stability and Control of Time-Delay Systems. Springer, 1998.

[10] Y. Y. Cao, Z. Lin, and T. Hu, "Stability analysis of linear time-delay systems subject to input saturation," IEEE Trans. Circuits Syst. I, Fundam. Theory Appl., vol. 49, pp. 233-240, 2002.

[11] B. S. Chen, S. S. Wang, and H. C. Lu, "Stabilization of time-delay systems containing saturating actuators," Int. J. Control, vol. 47, pp. 867-881, 1988.

[12] E. S. Cheres, S. Gutman, and Z. J. Palmor, "Stabilization of uncertain dynamic systems including state delay," IEEE Trans. Autom. Control, vol. 34, pp. 1199-1203, 1989. 
[13] S. C. Jeong and P. Park, "Constrained MPC algorithm for uncertain time-varying systems with state-delay," IEEE Trans. Autom. Control, vol. 50, pp. 257-263, 2005.

[14] B. M. Mirkin and P. Gutman, "Output feedback model reference adaptive control for multi-input multi-output plants with state delays," Syst. \& Control Lett., vol. 54, pp. 961-972, 2005.

[15] A. Alvarez-Aguirre, N. V. D. Wouw, T. Oguchi, and H. Nijmeijer, "Predictor-based remote tracking control of a movile robot," IEEE Trans. Control Syst. Tech., vol. 22, pp. 2087-2102, 2014.

[16] H. Hu, S. Y. Yoon, and Z. Lin, "Coordinated control of wheeled vehicles in the presence of a large communication delay through a potential functional approach," IEEE Trans. Intell. Transp. Syst., vol. 15, pp. 2261 - 2272, 2014.

[17] R. Piza, J. Salt, A. .Sala, and A. Cuenca, "Hierarchical triple-maglev dual-rate control over a profibus-dp network," IEEE Trans. Control Syst. Tech., vol. 22, pp. 1-12, 2014.

[18] O. M. Smith, "A controller to overcome deadtime," ISA Journal, vol. 6, pp. 28-33, 1959.

[19] Z. Artstein, "Linear systems with delayed controls: a reduction," IEEE Trans. Autom. Control, vol. 27, pp. 869-876, 1982.

[20] A. Z. Manitius and A. W. Olbrot, "Finite spectrum assignment problem for systems with delays," IEEE Trans. Autom. Control, vol. 24, pp. 541-553, 1979.

[21] N. Sharma, S. Bhasin, Q. Wang, and W. E. Dixon, "Predictor-based control for an uncertain Euler-Lagrange system with input delay," Automatica, vol. 47, pp. 2332$2342,2011$.

[22] M. Krstic, "Lyapunov stability of linear predictor feedback for time-varying input delay," IEEE Trans. Autom. Control, vol. 55, pp. 554-559, 2010.

[23] D. Bresch-Pietri and M. Krstic, "Delay-adaptive predictor feedback for systems with unknown long actuator delay," IEEE Trans. Autom. Control, vol. 55, pp. 2106-2112, 2010 .

[24] Z. Lin, Low Gain Feedback. London, UK: Springer-Verlag, 1988.

[25] S. Y. Yoon and Z. Lin, "Truncated predictor feedback control for exponentially unstable linear systems with time-varying input delay," Syst. Control Lett., vol. 62, pp. 837-844, 2013.

[26] R. Herzog, P. Buhler, C. Gahler, and R. Larsonneur, "Unbalance compensation using generalized notch filters in the multivariable feedback of magnetic bearings," IEEE Trans. Control Syst. Technol., vol. 4, pp. 580-586, 1996. 
[27] B. Shafai, S. Beale, P. LaRocca, and E. Cusson, "Magnetic bearing control systems and adaptive forced balancing," IEEE Contr. Syst. Mag., vol. 14, pp. 4-13, 1994.

[28] J. D. Setiawan, R. Mukherjee, and E. H. Maslen, "Adaptive compensation of sensor runout for magnetic bearings with uncertain parameters: theory and experiments," $J$. Dyn. Sys. Meas. Control, vol. 123, pp. 211-218, 2001.

[29] J. D. Setiawan, R. Mukherjee, and E. H. Maslen, "Synchronous sensor runout and unbalance compensation in active magnetic bearings using bias current excitation," $J$. Dyn. Sys. Meas. Control, vol. 124, pp. 14-24, 2002.

[30] C. Bi, D. Wu, Q. Jiang, and Z. Liu, "Automatic learning control for unbalance compensation in active magnetic bearings," IEEE Trans. Magn., vol. 41, pp. 2270-2280, 2005.

[31] I. S. Kuseyri, "Robust control and unbalance compensation of rotor/active magnetic bearing systems," J. Vib. Control, vol. 18, pp. 817-832, 2012.

[32] J. Tang, B. Liu, J. Fang, and S. S. Ge, "Suppression of vibration caused by residual unbalance of rotor for magnetically suspended flywheel," J. Vib. Control, vol. 19, pp. 1962-1979, 2013.

[33] Q. Chen, G. Liu, and S. Zheng, "Suppression of imbalance vibration for AMBs controlled driveline system using double loop structure," J. Sound Vib., vol. 337, pp. $1-13,2015$.

[34] E. H. Maslen, C. R. Knospe, and L. Zhu, "An enhanced dynamic model for the actuator/amplifier pair in AMB systems," in Proc. 10th Int. Symp. Magn. Bearings, pp. 395-399, 21-23 August 2006.

[35] P. F. Ribeiro, B. K. Johnson, M. L. Crow, A. Arsoy, and Y. Liu, "Energy storage systems for advanced power applications," Proceedings of the IEEE, vol. 89, no. 12, pp. 1744-1756, 2001.

[36] R. Hebner, J. Beno, and A. Walls, "Flywheel batteries come around again," IEEE Spectr., vol. 39, no. 4, pp. 46-51, 2002.

[37] G. O. Cimuca, C. Saudemont, B. Robyns, and M. M. Radulescu, "Control and performance evaluation of a flywheel energy-storage system associated to a variable-speed wind generator," IEEE Trans. Ind. Electron., vol. 53, no. 4, pp. 1074-1085, 2006.

[38] P. E. Kascak, T. P. Dever, and R. H. Jansen, "Magnetic circuit model of PM motorgenerator to predict radial forces," in: the 1st International Energy Conversion Engineering Conference, Cleveland, 2003.

[39] S. Y. Yoon, Z. Lin, T. Dimond, and P. E. Allaire, "Control of active magnetic bearing systems on non-static foundations," in: the 9th IEEE International Conference on Control and Automation (ICCA), pp. 556-561, Santiago, Chile, 2011. 
[40] M. I. Friswell, J. Penny, S. D. Garvey, and A. W. Lees, Dynamics of Rotating Machines. Cambridge: Cambridge University Press, 2010.

[41] S. Y. Yoon, P. Anantachaisilp, and Z. Lin, "An LMI approach to the control of exponentially unstable systems with input time delay," in: Proc. IEEE Conf. Decision and Control, pp. 312-317, Florence, Italy, 2013.

[42] B. A. Francis, "The linear multivariable regulator problem," SIAM J. Control Optim. vol. 15 , pp. 486-505, 1977.

[43] A. Isidori and C. I. Byrnes, "Output regulation of nonlinear systems," IEEE Trans. Autom. Control, vol. 35, pp. 131-140, 1990.

[44] C. I. Byrnes and A. Isidori, "Output regulation for nonlinear systems: an overview," Int. J. Robust Nonlin. Control, vol. 10, pp. 323-337, 2000.

[45] J. Huang, Nonlinear Output Regulation: Theory and Application, SIAM, Philadelphia, USA, 2004.

[46] B. Castillo-Toledo and E. Núñez-Pérez, "On the regulator problem for a class of LTI systems with delays," Kibernetika, vol. 39, no. 4, pp. 415-432, 2003.

[47] D. Wang, J. Wang, P. Shi, and W. Wang, "Output regulation of time delay systems based on internal model principle," in: Proc. 10th IEEE Int. Conf. Control Autom. (ICCA), pp. 1633-1638, Hangzhou, China, 2013.

[48] E. Fridman, "Output regulation of nonlinear systems with delay," Syst. \& Control Lett., vol. 50, pp. 81-93, 2003.

[49] M. Lu and J. Huang, "Robust output regulation problem for linear time-delay systems," Int. J. Control, vol. 88, no. 6, pp. 1236-1245, 2015.

[50] M. Lu and J. Huang, "Output regulation problem for linear time-delay systems," in: Proc. IEEE-CYBER 2014, pp. 274-279, 2014.

[51] S. Y. Yoon and Z. Lin, "Robust output regulation of linear time-delay systems: A state predictor approach," Int. J. Robust and Nonlinear Control, vol. 26, no. 8, pp. 1686-1704, 2015.

[52] Z. Zhang and A. Serrani, "The linear periodic output regulation problem," Syst. \&6 Control Lett., vol. 55, no. 7, pp. 518-529, 2005.

[53] A. Ichikawa and H. Katayama, "Output regulation of time-varying systems," Syst. \& Control Lett., vol. 55, no. 12, pp. 999-1005, 2006.

[54] H. Shim, J. H. Lee, J. S. Kim, and J. Back, "Output regulation problem and solution for LTV minimum phase systems with time-varying exosystem," In: Proc. of SICEICASE International joint conference, pp. 1823-1827, 2006. 
[55] H. Shim, J. S. Kim, H. Kim, and J. Back, "A note on the differential regulator equation for non-minimum phase linear systems with time-varying exosystems," Automatica, vol. 46, no. 3, pp. 605-609, 2010, .

[56] A. Isidori, Nonlinear Control Systems (Third ed.), Springer-Verlag, 1995.

[57] A. Isidori, L. Marconi, and A. Serrani, Robust Autonomous Guidance: An Internal Model Approach, Springer-Verlag, 2003.

[58] T. Hu, Z. Lin, and J. Lam, "Unified gradient approach to performance optimization under a pole assignment constraint," Journal of Optimization Theory and Application$s$, vol. 121, no. 2, pp. 361-383, 2004.

[59] P. Anantachaisilp, L. Di, S. Y. Yoon, and Z. Lin, "Control of active magnetic bearing systems with input delay for applications in remotely controlled turbomachinery," in: Proc. 53rd IEEE Conf. Decis. Control (CDC), Los Angeles, CA, 2014.

[60] A. Hassibi, J. How, and S. Boyd, "A path-following method for solving BMI problems in control," Proc. 1999 Am. Control Conf. (ACC), San Diego, CA, pp. 1385-1389, 1999.

[61] K. C. Toh, M. J. Todd, and R. H. Tutuncu, "SDPT3 - a Matlab software package for semidefinite programming," Optim. Methods Software, vol. 11, pp. 545-581, 1999.

[62] American Petroleum Institute, API 614: Axial and Centrifugal Compressors and Expander-compressors, 8th ed., 2014.

[63] International Organization for Standardization, ISO 14839-2: Mechanical vibration - Vibration of rotating machinery equipped with active magnetic bearings - Part 2: Evaluation of vibration, 2004.

[64] S. Y. Yoon, L. Di, and Z. Lin, "Unbalance Compensation for AMB Systems with Input Delay: an Output Regulation Approach," Control Engineering Practice (CEP), vol. 46, pp. 166-175, January 2016. 